\title{
Zeros de polinômios em espaços de Banach
}

\author{
Leandro Candido Batista
}

DissertaÇÃo APRESENTADA

$\mathrm{AO}$

Instituto De Matemática E Estatística

DA

Universidade de SÃo Paulo

PARA

OBTENÇÃO DO TÍTULO

$\mathrm{DE}$

Mestre em CiÊnCIAS

\author{
Programa: Matemática \\ Orientador: Profa. Dra. Mary Lilian Lourenço
}

Durante o desenvolvimento deste trabalho o autor recebeu auxílio financeiro do CNPq e da FAPESP (processo 2008/01650-0).

São Paulo, março de 2010 


\section{Polinômios n-Homogêneos e Tópicos Relacionados}

Este exemplar corresponde à redação final da dissertação devidamente corrigida e defendida por Leandro Candido Batista e aprovada pela Comissão Julgadora.

Banca Examinadora:

- Profa. Dra. Mary Lilian Lourenço - IME-USP.

- Prof. Dr. Daniel Pellegrino - UFPB.

- Prof. Dr. Leonardo Pellegrini - IME-USP. 

Aos meu pais. 


\section{Agradecimentos}

Agradeço à minha orientadora, Profa. Dra. Mary Lilian Lourenço, por sua orientação, sugestão dos temas, paciência e amizade.

Agradeço a meus pais Vera e Joaquim e meu irmão Leonardo, pelo apoio e incentivo.

Agradeço à minha noiva, Rita Cavalcanti, pelo carinho, paciência e apoio durante toda a graduação e o mestrado.

Agradeço a todos os professores que participaram da minha formação, em especial à Profa. Dra. Lúcia Renato Junqueira, por sua orientação, conselhos e amizade durante minha graduação.

Agradeço ao grande amigo André Pierro de Camargo que me ajudou de inúmeras formas.

Agradeço aos amigos Bárbara Sayuri Ashino e Cesar Adriano Batista pelo incentivo e auxílio com o latex.

Agradeço aos membros da banca examinadora pelas sugestões e correções.

Agradeço à FAPESP e ao CNPq pelo apoio financeiro. 


\section{Resumo}

Neste trabalho estudamos principalmente dois tópicos em Análise Funcional. No primeiro tópico, estudamos zeros de polinômios em espaços de Banach reais. Apresentamos resultados devidos a J. Ferrer, publicados em [11], estabelecendo que todo polinômio fracamente contínuo sobre os subconjuntos limitados de um espaço de Banach, de dual não separável na topologia fraca estrela, admite um subespaço linear fechado de dual não separável na topologia fraca estrela, no qual o polinômio se anula.

Apresentamos também uma demonstração, devida a J. Ferrer e publicada em [12], de que se $K$ é um espaço topológico compacto não satisfazendo a condição de cadeia contável, então todo polinômio definido em $\mathcal{C}(K)$ assumindo valores reais e se anulando na origem, se anula em um subespaço isometricamente isomorfo a $c_{0}(\Gamma)$, onde $\Gamma$ é um conjunto não enumerável.

No segundo tópico, exibimos uma versão multilinear para o Lema de Phelps, resultado publicado em [3] e devido a R. Aron, A. Cardwell., D. García e I. Zalzuendo. 


\section{Abstract}

We study two topics in Functional Analysis. In the first topic, we study zeros of polynomials on real Banach spaces. We present results due to J. Ferrer published in [11], stating that every polynomial weakly continuous on bounded subsets of a Banach space, whose dual is not separable in the weak-star topology, admits a closed linear subspace whose dual is not separable in the weak- star topology either, where the polynomial vanishes.

We also present a proof, given by J. Ferrer and published in [12], that if $K$ is a compact topological space not satisfying the countable chain condition, then every real-valued polynomial defined in $\mathcal{C}(K)$ and vanishing at the origin, vanishes in a subspace isometric to $c_{0}(\Gamma)$, where $\Gamma$ is a non-enumerable set.

In the second topic, we show a multilinear version for the Phelps' Lemma, published in [3] by R. Aron, A. Cardwell., D. García and I. Zalzuendo. 


\section{Introdução}

Neste trabalho estudamos principalmente dois tópicos em Análise Funcional. O primeiro e principal tópico é dedicado ao estudo de zeros de polinômios. O estudo de zeros de polinômios complexos possui uma longa história, com resultados em análise complexa, geometria algébrica e análise funcional. Um resultado devido a A. Plichko e A. Zagorodnyuk, publicado em [22], afirma que sobre um espaço de Banach complexo de dimensão infinita, todo polinômio $n$-homogêneo assumindo valores complexos se anula em um subespaço de dimensão infinita. Este resultado despertou grande interesse no estudo de zeros de polinômios $n$-homogêneos, em diversas direções.

Em [11], J. Ferrer estabelece condições sob as quais um resultado similar ao teorema de A. Plichko e A. Zagorodnyuk, descrito acima, seja válido para espaços de Banach reais de dimensão infinita. Este é um problema ainda não resolvido completamente. Neste artigo, o autor desenvolve técnicas que resolvem parcialmente este problema para um particular tipo de polinômio em um particular tipo de espaço.

No segundo tópico apresentaremos um resultado fora do contexto de zeros de polinômios, mas que nos despertou interesse durante o desenvolvimento desse trabalho. Se trata de uma versão multilinear para o Lema de Phelps, resultado publicado em [3] e devido a R. Aron, A. Cardwell., D. García e I. Zalzuendo.

O Lema de Phelps é um resultado devido a R. R. Phelps, publicado em 1960, em [20]. Em uma versão atual afirma que dados um espaço de Banach $X$, funcionais $f, g \in S_{X^{*}}$ e $0<\epsilon<1$, então

$$
S_{X} \cap \operatorname{ker} f \subset S_{X} \cap g^{-1}(]-\epsilon, \epsilon[) \Longrightarrow\|g-\alpha f\| \leq 2 \epsilon \text { para algum }|\alpha|=1 \text {. }
$$

No ano seguinte, este resultado foi utilizado em [6], por E. Bishop e R. R. Phelps, como um passo crucial na demonstração do teorema de Bishop-Phelps, que afirma que todo espaço de Banach é subreflexivo, ou seja, em um espaço de Banach o conjunto dos funcionais lineares contínuos que atingem sua norma é denso.

Em [3], os autores apresentam a seguinte versão multilinear para o Lema de Phelps.

Dados $X_{1}, \ldots, X_{n}$ espaços de Banach, para todo $n \in \mathbb{N}$ existe $D_{n}>0$ tal que para 
quaisquer $A, B \in \mathcal{L}\left(X_{1}, \ldots, X_{n}\right)$ com $\|A\|=\|B\|=1$ e $\epsilon>0$ suficientemente pequeno, definindo-se $Z(A):=\left\{u \in S_{X_{1}} \times \ldots \times S_{X_{n}}: A(u)=0\right\}$ e $\epsilon(B):=\left\{u \in S_{X_{1}} \times \ldots \times S_{X_{n}}\right.$ : $|B(u)| \leq \epsilon\}$

$$
Z(A) \subset \epsilon(B) \Rightarrow\|B-\alpha A\| \leq D_{n} \epsilon \text { para algum }|\alpha|=1
$$

A seguir, descreveremos sucintamente os assuntos abordados em cada capítulo.

No capítulo 1 estabelecemos resultados preliminares em análise funcional e topologia. Para um estudo detalhado sobre estes assuntos recomendamos [16].

No capítulo 2 introduzimos conceitos iniciais de aplicações multilineares e polinômios com base em [17]. Na seção 2.4 apresentamos, com base em [9], alguns tipos especiais de polinômios, dentre eles, os polinômios fracamente contínuos sobre subconjuntos limitados de um espaço de Banach, que terão um papel fundamental no capítulo 3.

No capítulo 3 estudamos zeros de polinômios em espaços de Banach reais. Apresentaremos, principalmente, resultados devidos a J. Ferrer publicados em [11] e [12]. Na seção 3.1 exibimos com todos os detalhes a demonstração do teorema de A. Plichko e A. Zagorodnyuk mencionado acima. Na seção 3.2 apresentamos alguns resultados e caracterizações para espaços de dual separável na topologia fraca estrela. Na seção 3.3 apresentamos a demonstração de que todo polinômio fracamente contínuo sobre os subconjuntos limitados de um espaço de Banach, de dual não separável na topologia fraca estrela, admite um subespaço linear fechado de dual não separável na topologia fraca estrela, no qual o polinômio se anula. Na seção 3.4, estudamos a aplicação de alguns resultados das seções anteriores sobre espaços com a propriedade de Dunford-Pettis. Apresentamos também a demonstração de que se $K$ é um espaço topológico compacto não satisfazendo a condição de cadeia contável, então todo polinômio definido em $\mathcal{C}(K)$ assumindo valores reais e se anulando na origem, se anula um um subespaço isometricamente isomorfo a $c_{0}(\Gamma)$, onde $\Gamma$ é um conjunto não enumerável.

No capítulo 4 nosso objetivo é exibir a versão multilinear do Lema de Phelps, elaborada por R. Aron, A. Cardwell., D. García e I. Zalzuendo, o que fazemos na seção 4.4.

Para um completa compreensão deste resultado são necessários alguns resultados encontrados nos artigos [5] e [18].

As seções 4.1 e 4.2 tratam de um pequeno estudo do atigo [18]. Estudamos técnicas de complexificação, que consistem em procedimentos para se obter espaços de Banach complexos a partir de espaços de Banach reais de forma que se possa estender aplicações multilineares e polinômios contínuos reais de forma única nesse espaço complexo, preservando-se a continuidade e controlando-se a norma. Na seção 4.3 estudamos, com base em [5], um método para, supondo $P_{1}, \ldots, P_{n}$ polinômios sobre um espaço de Banach $X$, se obter uma constante $M$ dependendo apenas dos graus de $P_{1}, \ldots, P_{n}$ satisfazendo $\left\|P_{1}\right\| \ldots\left\|P_{n}\right\| \leq M\left\|P_{1} \ldots P_{n}\right\|$. 


\section{Sumário}

Introdução $\quad$ ix

Notação $\quad$ xiii

1 Preliminares 1

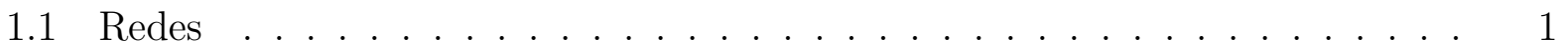

1.2 Topologias Induzidas por Famílias de Funções . . . . . . . . . . . . . . . . . 3

1.3 Espaços Vetoriais Topológicos . . . . . . . . . . . . . . . . . . . 10

1.4 Espaços de Sequências . . . . . . . . . . . . . . . . . . 12

2 Aplicações Multilineares e Polinômios $\quad 21$

2.1 Aplicações Multilineares . . . . . . . . . . . . . . . . . . . . . 21

2.2 Aplicações Multilineares Simétricas . . . . . . . . . . . . . . . . 27

2.3 Polinômios . . . . . . . . . . . . . . . . . . . . . . 31

2.4 Polinômios Fracamente Contínuos sobre Limitados . . . . . . . . . . . . . . 37

3 Zeros de Polinômios $\quad 43$

3.1 Zeros de Polinômios em Espaços de Banach Complexos . . . . . . . . . . . 43

3.2 Espaços de Dual $w^{*}$-Separável . . . . . . . . . . . . . . . . . . . . . . 47

3.3 Zeros de Polinômios Fracamente Contínuos sobre Limitados . . . . . . . . 53

3.4 Zeros de Polinômios sobre Espaços com a Propriedade DP . . . . . . . . . 58

4 Lema de Phelps Multilinear $\quad 67$

4.1 Complexificação de Espaços de Banach Reais . . . . . . . . . . . . . . . . . . 67

4.2 Extensões Complexas de Multilineares e Polinômios Reais . . . . . . . . . . . 73

4.3 Produto de Polinômios em Espaços de Banach . . . . . . . . . . . . . . 76

4.4 Lema de Phelps . . . . . . . . . . . . . . . . . . 85

Referências Bibliográficas $\quad 93$ 


\section{Notação}

\begin{tabular}{|c|c|}
\hline $\mathbb{N}$ & o conjunto dos números naturais \\
\hline $\mathbb{N}_{0}$ & o conjuntos dos números inteiros não negativos \\
\hline $\mathbb{Q}$ & o corpo dos racionais \\
\hline $\mathbb{R}$ & o corpo dos números reais \\
\hline $\mathbb{C}$ & o corpo dos números complexos \\
\hline $\mathbb{K}$ & o corpo $\mathbb{R}$ ou $\mathbb{C}$ \\
\hline $\mathcal{S}_{n}$ & o conjunto de todas as permutações de $\{1, \ldots, n\}$ \\
\hline$B_{X}$ & a bola unitária fechada de um espaço normado $X$ \\
\hline$S_{X}$ & a esfera unitária de um espaço normado $X$ \\
\hline $\mathcal{B}_{r}(x)$ & a bola aberta de centro $x$ e raio $r$ \\
\hline $\mathcal{L}(X ; Y)$ & o espaço das aplicações lineares contínuas de $X$ em $Y$ \\
\hline$X^{*}$ & o espaço das aplicações lineares contínuas de $X$ em $\mathbb{K}$ \\
\hline $\mathcal{L}\left({ }^{n} X ; Y\right)$ & o espaço das aplicações $n$-lineares contínuas de $X^{n}$ em $Y$ \\
\hline $\mathcal{L}\left({ }^{n} X\right)$ & o espaço das aplicações $n$-lineares contínuas de $X^{n}$ em $\mathbb{K}$ \\
\hline $\mathcal{P}\left({ }^{n} X ; Y\right)$ & o espaço dos polinômios $n$-homogêneos contínuos de $X$ em $Y$ \\
\hline $\mathcal{P}\left({ }^{n} X\right)$ & o espaço dos polinômios $n$-homogêneos contínuos de $X$ em $\mathbb{K}$ \\
\hline $\mathcal{C}(K)$ & o espaço das funções contínuas e limitadas de $K$ em $\mathbb{K}$ \\
\hline$[A]_{\mathbb{K}}$ & o espaço vetorial gerado por $A$ sobre o corpo $\mathbb{K}$ \\
\hline$[A]$ & quando não há dúvidas sobre o corpo, é o espaço vetorial gerado por $A$ \\
\hline$X \cong Y$ & $X, Y$ são espaços de Banach isometricamente isomorfos \\
\hline$\partial^{k} P$ & $\begin{array}{l}\text { é a aplicação em } \mathcal{P}\left({ }^{n-k} X ; \mathcal{P}\left({ }^{k} X ; Y\right)\right) \text {, definida por } \\
\partial^{k} P(x)(y):=\frac{n !}{(n-k) !} \widehat{P} x^{n-k} y^{k}, x, y \in X\end{array}$ \\
\hline$A^{\perp}$ & $\begin{array}{l}A \text { é subconjunto de um espaço normado } X, A^{\perp} \text { é o subespaço de } \\
\text { todos os funcionais } f \in X^{*} \text {, satistazendo } f(x)=0\end{array}$ \\
\hline$B_{\perp}$ & $\begin{array}{l}B \text { é subconjunto de } X^{*}, B_{\perp} \text { é o subespaço de todos os elementos } x \in X \\
\text { satistazendo } f(x)=0 \text {, para todo } f \in B\end{array}$ \\
\hline
\end{tabular}




\section{Capítulo 1}

\section{Preliminares}

Neste capítulo estudaremos conceitos básicos em topologia e análise funcional, necessários para o desenvolvimento deste trabalho. Para um estudo mais detalhado sobre esses tópicos recomendamos [16].

\section{$1.1 \quad$ Redes}

Em espaços métricos a continuidade de funções pode ser caracterizada através de sequências. Nesta seção introduziremos o conceito de rede, que generaliza o conceito de sequência e nos permite obter uma caracterização para a continuidade em espaços topológicos quaisquer.

Um estudo detalhado sobre redes bem como os resultados desta seção podem ser encontrados em [16].

Definição 1.1.1. Um conjunto não vazio $\Gamma$ munido de uma ordem parcial $\leq$, tal que para cada $\alpha, \beta \in \Gamma$ exista $\gamma \in \Gamma$ satisfazendo, $\alpha \leq \gamma$ e $\beta \leq \gamma$, é chamado conjunto dirigido. Uma rede em um conjunto $X$ é uma aplicação $x: \Gamma \rightarrow X$, onde $\Gamma$ é um conjunto dirigido. Denotaremos uma rede $x: \Gamma \rightarrow X$ por $\left(x_{\gamma}\right)_{\gamma \in \Gamma}$, onde $x_{\gamma}:=x(\gamma)$.

Dizemos que uma rede $\left(x_{\gamma}\right)_{\gamma \in \Gamma}$ em um espaço topológico $X$ converge para $x \in X$, se para qualquer vizinhança $U$ de $x$ existir $\gamma_{0} \in \Gamma$ tal que $x_{\gamma} \in U$, sempre que $\gamma \geq \gamma_{0}$. Demonstra-se que uma rede em um espaço de Hausdorff converge no máximo a um único elemento.

Neste trabalho, lidaremos apenas com espaços de Hausdorff. Portanto, se uma rede $\left(x_{\gamma}\right)_{\gamma \in \Gamma}$ converge a algum $x$, diremos que $x$ é o limite de $\left(x_{\gamma}\right)_{\gamma \in \Gamma}$ e denotaremos $x_{\gamma} \rightarrow x$ ou $\lim _{\gamma} x_{\gamma}=x$.

Claramente, toda sequência é uma rede.

A proposição a seguir nos fornece uma caracterização de fecho topológico por redes. 
Proposição 1.1.2. Seja $Y$ um subconjunto de um espaço topológico X. Então $x \in \bar{Y}$ se e somente se existe uma rede em $Y$ convergindo a $x$.

Demonstração. Claramente, se existe uma rede em $Y$ convergindo a $x$, então toda vizinhança de $x$ contém elementos desta rede e portanto $x \in \bar{Y}$.

Por outro lado, seja $x \in \bar{Y}$ e seja $\Gamma$ a coleção de todas as vizinhanças de $x$, dirigida sob a relação $\leq$, definida por

$$
U_{1} \leq U_{2} \text { se e somente se } U_{2} \subseteq U_{1}
$$

Para cada $U \in \Gamma$, fixemos $x_{U} \in U \cap Y$. Claramente $\left(x_{U}\right)_{U \in \Gamma}$ é uma rede em $Y$ convergindo a $x$.

O resultado a seguir nos fornece uma generalização, para espaços topológicos quaisquer, da caracterização de continuidade por sequências em espaços métricos.

Teorema 1.1.3. Sejam $X$ e $Y$ espaços topológicos. Uma função $f: X \rightarrow Y$ é contínua em $x_{0} \in X$ se e somente se a rede $\left(f\left(x_{\gamma}\right)\right)_{\gamma \in \Gamma}$ converge a $f\left(x_{0}\right)$, se $\left(x_{\gamma}\right)_{\gamma \in \Gamma}$ converge a $x_{0}$.

Demonstração. Se $f$ é contínua em $x_{0}$ e $\left(x_{\gamma}\right)_{\gamma \in \Gamma}$ é uma rede em $X$ convergindo a $x_{0}$, decorre imediatamente das definições de convergência e continuidade que $f\left(x_{\gamma}\right) \rightarrow f\left(x_{0}\right)$.

Por outro lado, supondo por absurdo que $f$ não seja contínua em $x_{0}$, existe uma vizinhança $V$ de $f\left(x_{0}\right)$ tal que nenhuma vizinhança $U$ de $x_{0}$ satisfaça $f(U) \subseteq V$.

Seja $\Gamma$ a coleção de todas as vizinhanças de $x_{0}$, dirigida sob a relação $\leq$, definida por

$$
U_{1} \leq U_{2} \text { se e somente se } U_{2} \subseteq U_{1}
$$

Para cada $U \in \Gamma$, seja $x_{U} \in U$ tal que $f\left(x_{U}\right) \notin V$. Decorre que $\left(x_{U}\right)_{U \in \Gamma}$ é uma rede em $X$ convergindo a $x_{0}$ e, por construção, $\left(f\left(x_{U}\right)\right)_{U \in \Gamma}$ não converge a $f\left(x_{0}\right)$, uma contradição.

De acordo com o teorema acima, uma função $f: X \rightarrow Y$ é contínua em $X$ se e somente se para cada $x \in X$, a rede $\left(f\left(x_{\gamma}\right)\right)_{\gamma \in \Gamma}$ converge a $f(x)$ se $\left(x_{\gamma}\right)_{\gamma \in \Gamma}$ converge a $x$.

Definição 1.1.4. Um subconjunto $\Gamma^{\prime}$ de um conjunto dirigido $\Gamma$ é cofinal em $\Gamma$ se para todo $\alpha \in \Gamma$ existir $\beta_{\alpha}$ em $\Gamma^{\prime}$ tal que $\alpha \leq \beta_{\alpha}$.

Definição 1.1.5. Sejam $X$ um conjunto, $\Gamma$ um conjunto dirigido e $\left(x_{\gamma}\right)_{\gamma \in \Gamma}$ uma rede em $X$. Sejam $\Gamma^{\prime}$ um conjunto dirigido e $g: \Gamma^{\prime} \rightarrow \Gamma$ uma função satisfazendo: 
(i) $g\left(\beta_{1}\right) \leq g\left(\beta_{2}\right)$ em $\Gamma$ sempre que $\beta_{1} \leq \beta_{2} \in \Gamma^{\prime}$.

(ii) $g\left(\Gamma^{\prime}\right)$ é cofinal em $\Gamma$.

Então, a rede $\left(x_{g(\beta)}\right)_{\beta \in \Gamma^{\prime}}$ é chamada subrede de $\left(x_{\gamma}\right)_{\gamma \in \Gamma}$.

Segue da definição acima que se $\left(x_{\gamma}\right)_{\gamma \in \Gamma}$ for uma rede em um conjunto $X$, então qualquer subrede de $\left(x_{\gamma}\right)_{\gamma \in \Gamma}$ é uma rede em $X$, e se $X$ for um espaço topológico e a rede $\left(x_{\gamma}\right)_{\gamma \in \Gamma}$ converge para algum $x \in X$, então toda subrede converge a $x$.

\subsection{Topologias Induzidas por Famílias de Funções}

Nesta seção introduziremos alguns resultados básicos em topologia geral e em espaços normados; introduziremos o conceito de topologias fraca e fraca*.

Os resultados desta seção se encontram em [16].

Teorema 1.2.1. Sejam $X$ um conjunto, $\mathcal{F}$ uma família de funções e $\left\{\left(X_{f}, \mathcal{O}_{f}\right): f \in \mathcal{F}\right\}$ uma família de espaços topológicos tal que cada $f$ em $\mathcal{F}$ seja função de $X$ em $X_{f}$. Então existe sobre $X$ uma topologia $\mathcal{O}$ satisfazendo:

(i) $f:(X, \mathcal{O}) \rightarrow\left(X_{f}, \mathcal{O}_{f}\right)$ é contínua para cada $f \in \mathcal{F}$;

(ii) se $\mathcal{O}^{\prime}$ for uma topologia sobre $X$ tal que $f:\left(X ; \mathcal{O}^{\prime}\right) \rightarrow\left(X_{f} ; \mathcal{O}_{f}\right)$ seja contínua para cada $f \in \mathcal{F}$, então $\mathcal{O} \subseteq \mathcal{O}^{\prime}$.

Denotaremos $\mathcal{O}:=\sigma(X, \mathcal{F})$.

Demonstração. Seja $\mathcal{G}=\left\{f^{-1}(U): f \in \mathcal{F}, U \in \mathcal{O}_{f}\right\}$ e $\mathcal{O}$ a topologia sobre $X$ gerada por $\mathcal{G}$. Claramente $f:(X, \mathcal{O}) \rightarrow\left(X_{f}, \mathcal{O}_{f}\right)$ é contínua para cada $f \in \mathcal{F}$.

Se $\mathcal{O}^{\prime}$ for uma outra topologia sobre $X$ tal que $f:\left(X, \mathcal{O}^{\prime}\right) \rightarrow\left(X_{f}, \mathcal{O}_{f}\right)$ seja contínua para cada $f \in \mathcal{F}$ então $\mathcal{G} \subseteq \mathcal{O}^{\prime}$ e consequentemente $\mathcal{O} \subseteq \mathcal{O}^{\prime}$.

Exemplo 1.2.2. (Topologia Produto) Dada uma coleção $\left\{X_{\gamma}: \gamma \in \Gamma\right\}$, definimos seu produto cartesiano por

$$
\prod_{\gamma \in \Gamma} X_{\gamma}:=\left\{f: \Gamma \rightarrow \bigcup_{\gamma \in \Gamma} X_{\gamma}: f(\gamma) \in X_{\gamma} \text { para todo } \gamma \in \Gamma\right\} .
$$

Denotaremos cada $f \in \prod_{\gamma \in \Gamma} X_{\gamma}$ por $\left(f_{\gamma}\right)_{\gamma \in \Gamma}$ onde $f_{\gamma}:=f(\gamma)$. 
Fixemos a coleção $\mathcal{F}=\left\{\pi_{\alpha}: \prod_{\gamma \in \Gamma} X_{\gamma} \rightarrow X_{\alpha}: \alpha \in \Gamma\right\}$ onde cada $\pi_{\alpha}$ é definido por,

$$
\pi_{\alpha}\left(\left(f_{\gamma}\right)_{\gamma \in \Gamma}\right):=f_{\alpha}
$$

A topologia em $\prod_{\gamma \in \Gamma} X_{\gamma}$, induzida pela coleção $\mathcal{F}$ é denominada topologia produto. Neste trabalho, sobre um produto cartesiano de espaços topológicos, sempre consideraremos a topologia produto.

Proposição 1.2.3. Sejam $X$ um conjunto, $\mathcal{F}$ uma coleção de funções satisfazendo as hipóteses do teorema 1.2.1 e $\left(x_{\gamma}\right)_{\gamma \in \Gamma}$ uma rede em $X$. Considerando sobre $X$ a topologia induzida pela coleção $\mathcal{F}$, então $x_{\gamma} \rightarrow x$ se e somente se $f\left(x_{\gamma}\right) \rightarrow f(x)$ para todo $f \in \mathcal{F}$.

Demonstração. É evidente que se $x_{\gamma} \rightarrow x$, então $f\left(x_{\gamma}\right) \rightarrow f(x)$ para todo $f \in \mathcal{F}$.

Por outro lado, fixada uma vizinhança arbitrária $U$ de $x$, existem $f_{1}, \ldots, f_{n} \in \mathcal{F}$ e $V_{k} \in \mathcal{O}_{f}, 1 \leq k \leq n$, tais que

$$
x \in f_{1}^{-1}\left(V_{1}\right) \cap \ldots \cap f_{n}^{-1}\left(V_{n}\right) \subseteq U
$$

Por $f\left(x_{\gamma}\right) \rightarrow f(x)$ para todo $f \in \mathcal{F}$, para cada $1 \leq k \leq n$, existe $\gamma_{k} \in \Gamma$, tal que $f_{k}\left(x_{\gamma}\right) \in V_{k}$ sempre que $\gamma \geq \gamma_{k}$.

Por $\Gamma$ ser um conjunto dirigido, existe $\gamma_{0} \in \Gamma$ tal que $\gamma_{0} \geq \gamma_{k}, 1 \leq k \leq n$. Decorre,

$$
x_{\gamma} \in f_{1}^{-1}\left(V_{1}\right) \cap \ldots \cap f_{n}^{-1}\left(V_{n}\right) \subseteq U,
$$

sempre que $\gamma \geq \gamma_{0}$. Portanto $x_{\gamma} \rightarrow x$.

Neste trabalho, se $X$ e $Y$ denotarem espaços vetoriais, $\mathcal{L}_{a}(X ; Y)$ denotará o espaço de todas as aplicações lineares de $X$ em $Y$. Em particular, se $Y=\mathbb{K}$ denotaremos $X_{a}^{*}:=$ $\mathcal{L}_{a}(X ; \mathbb{K})$. No caso em que $X$ e $Y$ forem espaços normados, denotaremos por $\mathcal{L}(X ; Y)$ o subespaço de todas as aplicações lineares contínuas de $X$ em $Y$. Em particular, se $Y=\mathbb{K}$, $X^{*}:=\mathcal{L}(X ; \mathbb{K})$.

Definição 1.2.4. (Topologia Fraca) Seja $X$ um espaço normado. A topologia induzida sobre $X$, pela coleção $X^{*}$ é denominada topologia fraca de $X$ e denotada $\sigma\left(X, X^{*}\right)$.

Por simplicidade, muitas vezes utilizaremos a letra $w$ fazendo referência à topologia fraca de um espaço normado. Por exemplo, dizemos que uma rede é $w$-convergente em um espaço normado $X$ se é convergente sob a topologia fraca de $X$. 
No que segue, apresentaremos alguns resultados relacionados à topologia fraca e que serão úteis neste trabalho.

Proposição 1.2.5. Se $T \in \mathcal{L}(X ; Y)$ então $T:\left(X, \sigma\left(X, X^{*}\right)\right) \rightarrow\left(Y, \sigma\left(Y, Y^{*}\right)\right)$ é contínua.

Demonstração. Fixemos $\left(x_{\gamma}\right)_{\gamma \in \Gamma}$ uma rede em $X$ convergindo fracamente a algum $x \in X$.

Para cada $\varphi \in Y^{*}$, temos $\varphi \circ T \in X^{*}$ e consequentemente

$$
\varphi\left(T\left(x_{\gamma}\right)\right)=\varphi \circ T\left(x_{\gamma}\right) \rightarrow \varphi \circ T(x)=\varphi(T(x)) .
$$

Em virtude da proposição 1.2.3, a rede $\left(T\left(x_{\gamma}\right)\right)_{\gamma \in \Gamma}$ converge fracamente a $T(x)$.

Por $\left(x_{\gamma}\right)_{\gamma \in \Gamma}$ ser uma rede $w$-convergente arbitrária, de acordo com a proposição 1.1.3, concluímos que $T:\left(X, \sigma\left(X, X^{*}\right)\right) \rightarrow\left(Y, \sigma\left(Y, Y^{*}\right)\right)$ é contínua.

A proposição a seguir nos permite simplificar algumas demonstrações. Sua verificação é simples e será omitida.

Proposição 1.2.6. Se $X$ é um espaço normado, então os conjuntos da forma

$$
W\left(x, \varphi_{1}, \ldots, \varphi_{n}, \epsilon\right):=\left\{y \in X:\left|\varphi_{k}(y)-\varphi_{k}(y)\right|<\epsilon, 1 \leq k \leq n\right\},
$$

onde $x \in X, \varphi_{1}, \ldots, \varphi_{n} \in X^{*} e \epsilon>0$, formam uma base de abertos para a topologia $\sigma\left(X, X^{*}\right)$.

Definição 1.2.7. A aplicação $\mathcal{I}: X \rightarrow X^{* *}$ que associa a cada $x \in X$, o funcional $\mathcal{I}_{x} \in X^{* *}$ definido por

$$
\mathcal{I}_{x}(\varphi):=\varphi(x), x \in X, \varphi \in X^{*},
$$

é chamada de aplicação canônica de $X$ em $X^{* *}$.

Utilizando-se o teorema de Hahn-Banach, demonstra-se que $\mathcal{I}$ é uma isometria linear.

Definição 1.2.8. Se $X$ é um espaço normado, dizemos que um subconjunto $A \subseteq X$ é fracamente limitado se para cada $\varphi \in X^{*}$, existe $M_{\varphi}>0$ tal que $\sup _{x \in A}|\varphi(x)|<M_{\varphi}$.

Proposição 1.2.9. Se $X$ é um espaço normado, um subconjunto de $X$ é limitado se e somente se for fracamente limitado.

Demonstração. Seja $A$ um subconjunto de $X$. Se $A$ for limitado, existe $M>0$ satisfazendo

$$
\sup _{x \in A}\|x\|<M
$$


Para cada $\varphi \in X^{*}$, fixando $M_{\varphi}:=\|\varphi\| M$, decorre

$$
\sup _{x \in A}|\varphi(x)| \leq \sup _{x \in A}\|\varphi\|\|x\|<\|\varphi\| M=M_{\varphi}
$$

Concluímos que $A$ é fracamente limitado.

Por outro lado, se $A$ é fracamente limitado e $\mathcal{I}: X \rightarrow X^{* *}$ é a aplicação canônica, então $\left\{\mathcal{I}_{x}: x \in A\right\}$ é uma coleção em $X^{* *}$, pontualmente limitada. O Princípio da Limitação Uniforme implica a existência de $M>0$ satisfazendo

$$
\sup _{x \in A}\|x\|=\sup _{x \in A}\left\|\mathcal{I}_{x}\right\|<M
$$

Portanto $A$ é limitado.

Definição 1.2.10. (Topologia Fraca* $^{*}$ ) Sejam $X$ um espaço normado e $\mathcal{I}: X \rightarrow X^{* *}$ a aplicação canônica. A topologia induzida sobre $X^{*}$, pela coleção $\mathcal{I}(X) \subseteq X^{* *}$ é denominada topologia fraca* de $X^{*}$ e denotada $\sigma\left(X^{*}, X\right)$.

Analogamente à topologia fraca, muitas vezes utilizaremos $w^{*}$ fazendo referência à topologia fraca* no dual de um espaço normado.

No que segue, apresentaremos alguns resultados relacionados à topologia fraca* e que serão úteis neste trabalho.

A proposição a seguir é uma aplicação simples do teorema 1.1.3.

Proposição 1.2.11. Se X é um espaço normado, então a aplicação canônica

$$
\mathcal{I}:(X,\|\cdot\|) \rightarrow\left(X^{* *}, \sigma\left(X^{*}, X\right)\right)
$$

é contínua.

Analogamente à proposição 1.2.6, a proposição a seguir nos permite simplificar algumas demonstrações. A demonstração é simples e será omitida.

Proposição 1.2.12. Se $X$ é um espaço normado, então os conjuntos da forma

$$
W\left(\varphi, x_{1}, \ldots, x_{n}, \epsilon\right):=\left\{\vartheta \in X^{*}:\left|\varphi\left(x_{k}\right)-\vartheta\left(x_{k}\right)\right|<\epsilon, 1 \leq k \leq n\right\}
$$

onde $\varphi \in X^{*}, x_{1}, \ldots, x_{n} \in X e \epsilon>0$, formam uma base de abertos para a topologia $\sigma\left(X^{*}, X\right)$. 
Dado um espaço topológico $X$, dizemos que um subconjunto $Y \subset X$ é separável se existir um subconjunto $\mathcal{D} \subset Y$ enumerável, satisfazendo $Y \subseteq \overline{\mathcal{D}}$.

Se $X$ é um espaço normado e $A$ e $B$ forem subconjuntos de $X$ e $X^{*}$ respectivamente, denotaremos por $\bar{A}^{w}$ o fecho de $A$ sob a topologia fraca de $X$ e por $\bar{B}^{w *}$ o fecho de $B$ sob a topologia fraca* de $X^{*}$.

Demonstra-se que se um subconjunto $Y \subseteq X$, onde $X$ é um espaço normado, é separável então $[Y]$ é separável. Temos também o seguinte resultado.

Proposição 1.2.13. Seja $X$ um espaço normado. Se um subconjunto $A$ de $X^{*}$ é $w^{*}$ separável, então $[A]$ é $w^{*}$-separável.

Demonstração. Se $A$ é $w^{*}$-separável, existe uma coleção enumerável $\left\{\varphi_{n}: n \in \mathbb{N}\right\} \subset A$ satisfazendo

$$
A \subseteq{\overline{\left\{\varphi_{n}: n \in \mathbb{N}\right\}}}^{w^{*}}
$$

Seja $\mathbb{K}_{\mathbb{Q}}$ definido por

$$
\mathbb{K}_{\mathbb{Q}}:=\left\{\begin{array}{ll}
\mathbb{Q} & \text { se } \mathbb{K}=\mathbb{R} \\
\mathbb{Q}+i \mathbb{Q} & \text { se } \mathbb{K}=\mathbb{C}
\end{array} .\right.
$$

Claramente $\mathbb{K}_{\mathbb{Q}}$ é um corpo denso em $\mathbb{K}$ e podemos definir $\mathcal{D}:=\left[\left\{\varphi_{n}: n \in \mathbb{N}\right\}\right]_{\mathbb{K}_{\mathbb{Q}}}$, o espaço vetorial gerado por $\left\{\varphi_{n}: n \in \mathbb{N}\right\}$ sobre o corpo $\mathbb{K}_{\mathbb{Q}}$. Segue-se que $\mathcal{D}$ é enumerável. Demonstraremos que $[A] \subset \overline{\mathcal{D}}^{w^{*}}$.

Fixado $u \in[A]$, seja $W\left(u, x_{1}, \ldots, x_{r}, \epsilon\right)$, onde $x_{1}, \ldots, x_{r} \in X$, e $\epsilon>0$, uma vizinhança básica arbitrária de $u$ na topologia fraca* de $X^{*}$.

Supondo $u=a_{1} \vartheta_{1}+\ldots+a_{s} \vartheta_{s}, \operatorname{com} a_{1}, \ldots, a_{s} \in \mathbb{K}$ e $\vartheta_{1}, \ldots, \vartheta_{s} \in A$, por $\left\{\varphi_{k}: k \in \mathbb{N}\right\}$ ser $w^{*}$-denso em $A$, para cada $1 \leq k \leq s$ existe $\varphi_{n_{k}} \in W\left(\vartheta_{k}, x_{1}, \ldots, x_{r}, \delta\right) \cap\left\{\varphi_{n}: n \in \mathbb{N}\right\}$, onde

$$
\delta:=\frac{\epsilon}{2 s\left(\max _{1 \leq j \leq s}\left|a_{j}\right|+1\right)} .
$$

Então, para cada $1 \leq k \leq s$,

$$
\left|\vartheta_{k}\left(x_{j}\right)-\varphi_{n_{k}}\left(x_{j}\right)\right|<\frac{\epsilon}{2 s\left(\max _{1 \leq j \leq s}\left|a_{j}\right|+1\right)}, 1 \leq j \leq r .
$$

Fixando-se $v:=a_{1} \varphi_{n_{1}}+\ldots+a_{s} \varphi_{n_{s}}$, a densidade de $\mathbb{K}_{\mathbb{Q}}$ em $\mathbb{K}$ implica a existência de $b_{1}, \ldots, b_{s} \in \mathbb{K}_{\mathbb{Q}}$, satisfazendo

$$
\left|a_{k}-b_{k}\right|<\frac{\epsilon}{2 s\left(\max _{1 \leq j \leq r}\left|\varphi_{n_{k}}\left(x_{j}\right)\right|+1\right)}, 1 \leq k \leq s .
$$


Segue-se que $w:=b_{1} \varphi_{n_{1}}+\ldots+b_{r} \varphi_{n_{r}} \in \mathcal{D}$ e para cada $1 \leq j \leq r$,

$$
\begin{aligned}
\left|u\left(x_{j}\right)-w\left(x_{j}\right)\right| & \leq\left|u\left(x_{j}\right)-v\left(x_{j}\right)\right|+\left|v\left(x_{j}\right)-w\left(x_{j}\right)\right| \\
& =\left|\sum_{k=1}^{s} a_{k}\left(\vartheta_{k}\left(x_{j}\right)-\varphi_{n_{k}}\left(x_{j}\right)\right)\right|+\left|\sum_{k=1}^{s}\left(a_{k}-b_{k}\right) \varphi_{n_{k}}\left(x_{j}\right)\right| \\
& \leq \sum_{k=1}^{s}\left|a_{k}\right|\left|\vartheta_{k}\left(x_{j}\right)-\varphi_{n_{k}}\left(x_{j}\right)\right|+\sum_{k=1}^{s}\left|a_{k}-b_{k}\right|\left|\varphi_{n_{k}}\left(x_{j}\right)\right| \\
& <\sum_{k=1}^{s} \frac{\epsilon}{2 s}+\sum_{k=1}^{s} \frac{\epsilon}{2 s} \\
& =\frac{\epsilon}{2}+\frac{\epsilon}{2}=\epsilon .
\end{aligned}
$$

Então $w \in W\left(u, x_{1}, \ldots, x_{r}, \epsilon\right) \cap \mathcal{D}$. Por $W\left(u, x_{1}, \ldots, x_{r}, \epsilon\right)$ ser uma vizinhança básica arbitrária de $u$, concluímos que $u \in \overline{\mathcal{D}}^{w^{*}}$.

Apresentaremos agora algumas definições e teoremas importantes que serão utilizados ao longo deste trabalho. Estes resultados podem ser encontrados com todos os detalhes em [16].

Definição 1.2.14. Sejam $X$ um espaço normado e $A$ e $B$ subconjuntos de $X$ e $X^{*}$ respectivamente, definimos

$$
A^{\perp}:=\left\{f \in X^{*}: f(x)=0, x \in A\right\}, B_{\perp}:=\{x \in X: f(x)=0, f \in B\} .
$$

$A^{\perp}$ é o anulador de $A$ em $X^{*}$ e $B_{\perp}$ é o anulador de $B$ em $X$.

Demonstra-se que $A^{\perp}$ e $B_{\perp}$ são subespaços fechados de $X^{*}$ e $X$ respectivamente.

Teorema 1.2.15. Sejam $X$ um espaço normado e $A$ e $B$ subconjuntos de $X$ e $X^{*}$ respectivamente.

(i) O conjunto $A^{\perp}$ é $w^{*}$-fechado em $X$;

(ii) $\left(B_{\perp}\right)^{\perp}=\overline{[B]}^{w^{*}}$;

(iii) Se B for subespaço de $X^{*}$, então $\left(B_{\perp}\right)^{\perp}=\bar{B}^{w^{*}}$.

Teorema 1.2.16. (Alaoglu) Se X é um espaço normado, então a bola fechada $B_{X^{*}}$ é um espaço de Hausdorff compacto na topologia fraca*.

Teorema 1.2.17. (Goldstine) Sejam $X$ um espaço normado e $\mathcal{I}: X \rightarrow X^{* *}$ a aplicação canônica. Então $\mathcal{I}(X)$ é $w^{*}$-denso em $X^{* *}$. 
Teorema 1.2.18. Se $X$ é um espaço normado separável então $B_{X^{*}}$ é w*-metrizável.

Demonstração. Seja $\left\{x_{n}: n \in \mathbb{N}\right\} \subset X \backslash\{0\}$ um subconjunto enumerável, denso em $X$. Observamos que para quaisquer $f, g \in B_{X^{*}}$

$$
\sum_{n=1}^{\infty} \frac{1}{2^{n}\left\|x_{n}\right\|}\left|(f-g)\left(x_{n}\right)\right| \leq \sum_{n=1}^{\infty} \frac{1}{2^{n}\left\|x_{n}\right\|}\|f-g\|\left\|x_{n}\right\|=\|f-g\|<\infty .
$$

Podemos então definir a aplicação d : $B_{X^{*}} \times B_{X^{*}} \rightarrow \mathbb{R}$ por

$$
\mathrm{d}(f, g):=\sum_{n=1}^{\infty} \frac{1}{2^{n}\left\|x_{n}\right\|}\left|(f-g)\left(x_{n}\right)\right|, f, g \in B_{X^{*}}
$$

Verifica-se sem dificuldade que para quaisquer $f, g, h \in B_{X^{*}}$

(i) $\mathrm{d}(f, g) \geq 0$;

(ii) $\mathrm{d}(f, g)=\mathrm{d}(g, f)$;

(iii) $\mathrm{d}(f, g) \leq \mathrm{d}(f, h)+\mathrm{d}(h, g)$.

Se $f, g \in B_{X^{*}}$ são tais que $\mathrm{d}(f, g)=0$, então $f\left(x_{n}\right)=g\left(x_{n}\right)$ para todo $n \in \mathbb{N}$. Por $\left(x_{n}\right)_{n \in \mathbb{N}}$ ser denso em $X$, decorre $f=g$. Podemos então concluir que d é uma métrica sobre $B_{X^{*}}$.

Para demonstrarmos que a topologia $\sigma\left(X^{*}, X\right)$ sobre $B_{X^{*}}$ é metrizável, basta verificarmos que a identidade id : $\left(B_{X^{*}}, \sigma\left(X^{*}, X\right)\right) \rightarrow\left(B_{X^{*}}, \mathrm{~d}\right)$ é um homeomorfismo.

Seja $f \in B_{X^{*}}$ arbitrário. Dado $\epsilon>0$ arbitrário, existe $n_{0} \in \mathbb{N}$ tal que $\frac{1}{2^{n_{0}}}<\frac{\epsilon}{4}$ e existe $\delta>0$ tal que

$$
\delta\left(\sum_{k=1}^{n_{0}} \frac{1}{2^{n}\left\|x_{n}\right\|}\right)<\frac{\epsilon}{2} .
$$

Seja $W:=W\left(f, x_{1}, \ldots, x_{n_{0}}, \delta\right) \cap B_{X^{*}}$. Se $g \in W$ temos

$$
\begin{aligned}
\mathrm{d}(f, g) & =\sum_{k=1}^{n_{0}} \frac{1}{2^{k}\left\|x_{k}\right\|}\left|(f-g)\left(x_{k}\right)\right|+\sum_{k=n_{0}+1}^{\infty} \frac{1}{2^{k}\left\|x_{k}\right\|}\left|(f-g)\left(x_{k}\right)\right| \\
& \leq \delta\left(\sum_{k=1}^{n_{0}} \frac{1}{2^{k}\left\|x_{k}\right\|}\right)+\|f-g\|\left(\sum_{k=n_{0}+1}^{\infty} \frac{1}{2^{k}}\right) \\
& \leq \frac{\epsilon}{2}+2\left(\sum_{k=n_{0}+1}^{\infty} \frac{1}{2^{k}}\right) \\
& \leq \frac{\epsilon}{2}+\frac{2}{2^{n_{0}}}<\epsilon .
\end{aligned}
$$


Isso demonstra que id $(W) \subseteq \mathcal{B}_{\epsilon}^{\mathrm{d}}(f):=\left\{g \in B_{X^{*}}: \mathrm{d}(f, g)<\epsilon\right\}$, e podemos concluir que id é contínua. Por outro lado, de acordo com o teorema de Alaoglu 1.2.16, $B_{X^{*}}$ é $w^{*}$-compacto e por $\left(B_{X^{*}}, \mathrm{~d}\right)$ ser espaço topológico de Hausdorff, id é uma aplicação fechada. Segue-se que id $^{-1}$ é contínua e concluímos que id é um homeomorfismo.

\subsection{Espaços Vetoriais Topológicos}

Nesta seção introduziremos o conceito de espaço vetorial topológico que generaliza o de espaço normado. Apresentaremos algumas definições e resultados que dependem apenas da continuidade das operações de adição de vetores e multiplicação de vetor por escalar. Estudaremos também o conceito de precompacidade.

Definição 1.3.1. Um espaço vetorial topológico (EVT) é um par ordenado $(X, \mathcal{O})$, onde $X$ é um espaço vetorial sobre o corpo $\mathbb{K}$ e $\mathcal{O}$ é uma topologia sobre $X$ tal que a adição de vetores seja uma operação contínua de $X \times X$ em $X$ e a multiplicação de vetor por escalar seja uma operação contínua de $\mathbb{K} \times X$ em $X$. Se $\mathcal{O}$ admitir uma base de abertos convexos, dizemos que $(X, \mathcal{O})$ é um espaço localmente convexo (ELC). Por questão de simplicidade, quando não houver dúvidas sobre qual topologia se trata, denotaremos um espaço vetorial topológico apenas por $X$.

Claramente, todo espaço normado $X$ é um ELC. Verifica-se também que os espaços $\left(X, \sigma\left(X, X^{*}\right)\right)$ e $\left(X^{*}, \sigma\left(X^{*}, X\right)\right)$ são ELCs.

Definição 1.3.2. Uma rede $\left(x_{\gamma}\right)_{\gamma \in \Gamma}$ em um espaço vetorial topológico $X$ é chamada de rede de Cauchy se para toda vizinhança $U$ de zero existir $\gamma \in \Gamma$ tal que $x_{\alpha}-x_{\beta} \in U$ sempre que $\alpha, \beta \geq \gamma$.

Definição 1.3.3. Dados $X, Y$ espaços vetoriais topológicos, dizemos que que uma aplicação $f: A \subset X \rightarrow Y$ é uniformemente contínua se para toda vizinhança $U$ de zero em $Y$ existir uma vizinhança $V$ de zero em $X$, tal que para quaisquer $x, y \in A, f(x)-f(y) \in U$ sempre que $x-y \in V$.

Definição 1.3.4. Se $X$ é um espaço vetorial topológico e $Y$ é subconjunto de $X$, dizemos que $Y$ é precompacto se toda rede $\left(x_{\gamma}\right)_{\gamma \in \Gamma} \subset Y$ admite subrede de Cauchy.

Sejam $X, Y$ são espaços vetoriais topológicos e $A \subset X$ precompacto. Se $f: A \rightarrow Y$ for uma aplicação não uniformemente contínua, de acordo com a definição 1.3.3, existe uma 
vizinhança $U$ de zero em $Y$ tal que para cada vizinhança $V$ de zero em $X$, existem $x_{V}$, $y_{V} \in A$, satisfazendo

$$
x_{V}-y_{V} \in V \text { e } f\left(x_{V}\right)-f\left(y_{V}\right) \notin U .
$$

Seja $\Gamma$ a coleção de todas as vizinhanças de zero, dirigida sob a relação $\leq$, definida por

$$
V_{0} \leq V_{1} \text { se e somente se se } V_{1} \subseteq V_{0} .
$$

Segue-se que $\left(x_{V}\right)_{V \in \Gamma}$ e $\left(y_{V}\right)_{V \in \Gamma}$ são redes em $A$ satisfazendo, $x_{V}-y_{V} \rightarrow 0$.

Por $A$ ser precompacto, $\left(x_{V}\right)_{V \in \Gamma}$ e $\left(y_{V}\right)_{V \in \Gamma}$ admitem subredes de Cauchy que, por simplicidade, denotaremos $\left(x_{\gamma}\right)_{\gamma \in \Gamma_{1}}$ e $\left(y_{\delta}\right)_{\delta \in \Gamma_{2}}$ respectivamente.

Seja $\Gamma_{0}:=\Gamma_{1} \times \Gamma_{2}$, dirigido sob a relação $\leq$, definida por

$$
\left(\gamma_{0}, \delta_{0}\right) \leq\left(\gamma_{1}, \delta_{1}\right) \text { se e somente se } \gamma_{0} \leq \gamma_{1} \text { e } \delta_{0} \leq \delta_{1}
$$

Fixando-se $\left(x_{(\gamma, \delta)}\right)_{(\gamma, \delta) \in \Gamma_{0}}$ e $\left(y_{(\gamma, \delta)}\right)_{(\gamma, \delta) \in \Gamma_{0}}$ onde

$$
x_{(\gamma, \delta)}:=x_{\gamma} \text { e } y_{(\gamma, \delta)}:=y_{\delta},
$$

obtemos redes de Cauchy em $A$, satisfazendo

$$
x_{(\gamma, \delta)}-y_{(\gamma, \delta)} \rightarrow 0 \text { e } f\left(x_{(\gamma, \delta)}\right)-f\left(y_{(\gamma, \delta)}\right) \notin U, \quad(\gamma, \delta) \in \Gamma_{0} .
$$

Proposição 1.3.5. Se $X$ e $Y$ forem espaços localmente convexos e $A \subset X$ for precompacto, então uma aplicação $f: A \rightarrow Y$ é uniformemente contínua se e somente se aplica redes de Cauchy em redes de Cauchy.

Demonstração. Claramente se $f$ é uniformemente contínua então aplica redes de Cauchy em redes de Cauchy.

Por outro lado, se $f$ não for uniformemente contínua, de acordo com o que foi discutido acima, deve existir uma vizinhança $U$ de zero e redes de Cauchy $\left(x_{\gamma}\right)_{\gamma \in \Gamma}$ e $\left(y_{\gamma}\right)_{\gamma \in \Gamma}$ em $A$, satisfazendo

$$
x_{\gamma}-y_{\gamma} \rightarrow 0 \text { e } f\left(x_{\gamma}\right)-f\left(y_{\gamma}\right) \notin U, \gamma \in \Gamma .
$$

Seja $\Gamma_{\mathbb{N}}:=\Gamma \times \mathbb{N}$, dirigido sob a relação $\leq$, definida por

$$
\left(\gamma_{1}, n_{1}\right) \leq\left(\gamma_{2}, n_{2}\right) \quad \text { se e somente se } \gamma_{1} \leq \gamma_{2} \text { e } n_{1} \leq n_{2} .
$$


Fixemos $\left(z_{(\gamma, n)}\right)_{(\gamma, n) \in \Gamma_{\mathbb{N}}}$ definida por

$$
z_{(\gamma, n)}= \begin{cases}x_{\gamma} & \text { se } \mathrm{n} \text { for par } \\ y_{\gamma} & \text { se } \mathrm{n} \text { for ímpar }\end{cases}
$$

Sem dificuldades verifica-se que $\left(z_{(\gamma, n)}\right)_{(\gamma, n) \in \Gamma_{\mathbb{N}}}$ é rede de Cauchy em $A$. Entretanto, a rede $\left(f\left(z_{(\gamma, n)}\right)\right)_{(\gamma, n) \in \Gamma_{\mathbb{N}}}$ não é de Cauchy em $Y$, isso conclui a demonstração.

Proposição 1.3.6. Se X é um espaço de Banach, todo subconjunto precompacto de $X$ é separável.

Demonstração. Seja $A$ um subconjunto precompacto de $X$. Verifica-se que toda sequência em $\bar{A}$ admite subsequência convergindo a algum elemento de $\bar{A}$, portanto $\bar{A}$ é compacto.

Por $X$ ser um espaço de Banach e $\bar{A}$ ser compacto, $\bar{A}$ é separável, e portanto existe um subconjunto enumerável $\mathcal{D}=\left\{a_{n}: n \in \mathbb{N}\right\}$ satisfazendo

$$
\bar{A}=\overline{\mathcal{D}}
$$

Para cada $m, n \in \mathbb{N}$, fixemos $b_{m n} \in \mathcal{B}_{\frac{1}{m}}\left(a_{n}\right) \cap A$. Segue-se que $\mathcal{H}:=\left\{b_{m n}: m, n \in \mathbb{N}\right\} \subset A$ é uma coleção enumerável, satisfazendo

$$
A \subseteq \overline{\mathcal{H}}
$$

de onde concluímos que $A$ é separável.

A demonstração da proposição a seguir pode ser encontrada com todos os detalhes em [16] ou [19].

Proposição 1.3.7. Se $X$ é um espaço de Banach então $B_{X}$ é w-precompacto.

\subsection{Espaços de Sequências}

Nesta seção introduziremos uma importante classe de espaços que será utilizada durante todo o trabalho. Para maiores detalhes sobre este tópico, recomendamos [10]. 
Fixado um conjunto infinito $\Gamma, c_{0}(\Gamma)$ denota o espaço de todas as funções $\varphi: \Gamma \rightarrow \mathbb{K}$ satisfazendo

$$
\|\varphi\|_{\infty}:=\sup _{\lambda \in \Gamma}\{|\varphi(\lambda)|\}<\infty .
$$

e tais que para todo $\epsilon>0$ o conjunto $\{\lambda \in \Gamma:|\varphi(\lambda)| \geq \epsilon\}$ é finito.

Observamos que sob esta definição, qualquer $\varphi \in c_{0}(\Gamma)$ se anula em todo $\Gamma$, com exceção de um subconjunto enumerável $\Gamma_{\varphi}$.

Se $1 \leq p<\infty, l_{p}(\Gamma)$ denota o conjunto de todas as funções $\varphi: \Gamma \rightarrow \mathbb{K}$ se anulando em todo $\Gamma$ com exceção de um subconjunto enumerável $\Gamma_{\varphi} \subseteq\left\{\lambda_{k}: k \in \mathbb{N}\right\}$ satisfazendo

$$
\|\varphi\|_{p}:=\left(\sum_{k=1}^{\infty}\left|\varphi\left(\lambda_{k}\right)\right|^{p}\right)^{\frac{1}{p}}<\infty .
$$

Por abuso de notação muitas vezes escreveremos

$$
\|\varphi\|_{p}:=\left(\sum_{\lambda \in \Gamma}|\varphi(\lambda)|^{p}\right)^{\frac{1}{p}}
$$

Denotamos por $l_{\infty}(\Gamma)$ o espaço de todas as funções $\varphi: \Gamma \rightarrow \mathbb{K}$ com

$$
\|\varphi\|_{\infty}:=\sup _{t \in \Gamma}\{|\varphi(t)|\}
$$

Demonstra-se que $c_{0}(\Gamma)$ e $l_{p}(\Gamma), 1 \leq p \leq \infty$ são espaços de Banach. Um elemento arbitrário $\varphi$ de algum desses espaços, será denotado por $\left(\varphi_{\lambda}\right)_{\lambda \in \Gamma}$ onde $\varphi_{\lambda}:=\varphi(\lambda)$.

Por simplicidade, denotaremos $c_{0}(\mathbb{N}):=c_{0}$ e $l_{p}(\mathbb{N}):=l_{p}, 1 \leq p \leq \infty$.

Seja $\mathcal{B}=\left\{e_{\lambda}: \lambda \in \Gamma\right\}$ onde $e_{\lambda}=\left(e_{\lambda \gamma}\right)_{\gamma \in \Gamma}$ é definido por

$$
e_{\lambda \gamma}:= \begin{cases}1 & \text { se } \lambda=\gamma \\ 0 & \text { se } \lambda \neq \gamma\end{cases}
$$

Claramente $\mathcal{B}$ é um conjunto linearmente independente. Fixado $\varphi=\left(\varphi_{\lambda}\right)_{\lambda \in \Gamma}$ em $l_{p}(\Gamma)$, $1 \leq p<\infty$ ou $c_{0}(\Gamma)$, o conjunto $\Gamma_{\varphi}:=\left\{\lambda \in \Gamma: \varphi_{\lambda} \neq 0\right\}$ é enumerável.

Se $\Gamma_{\varphi}$ for finito, $\varphi$ é combinação linear de elementos de $\mathcal{B}$. Supondo $\Gamma_{\varphi}=\left\{\lambda_{k}: k \in \mathbb{N}\right\}$, definimos a sequência $\left(\vartheta_{n}\right)_{n \in \mathbb{N}}$ por

$$
\vartheta_{n}:=\sum_{k=1}^{n} \varphi_{\lambda_{k}} e_{\lambda_{k}}
$$


Dado $\epsilon>0$, se $\varphi \in l_{p}(\Gamma), 1 \leq p<\infty$, existe $n_{0}$ tal que

$$
\left\|\varphi-\vartheta_{n}\right\|_{p} \leq\left(\sum_{k=n+1}^{\infty}\left|\varphi_{\lambda_{k}}\right|^{p}\right)^{\frac{1}{p}}<\epsilon
$$

sempre que $n \geq n_{0}$.

Se $\varphi \in c_{0}(\Gamma)$, existe $n_{1}$ tal que

$$
\left\|\varphi-\vartheta_{n}\right\|_{\infty} \leq \sup _{k \geq n+1}\left\{\left|\varphi_{\lambda_{k}}\right|\right\} \leq \epsilon
$$

sempre que $n \geq n_{1}$.

Em qualquer caso

$$
\varphi=\lim _{n \rightarrow \infty} \vartheta_{n}=\lim _{n \rightarrow \infty} \sum_{k=1}^{n} \varphi_{\lambda_{k}} e_{\lambda_{k}}:=\sum_{k=1}^{\infty} \varphi_{\lambda_{k}} e_{\lambda_{k}}
$$

Claramente, a série converge incondicionalmente, ou seja

$$
\sum_{k=1}^{\infty} \varphi_{\lambda_{k}} e_{\lambda_{k}}=\sum_{k=1}^{\infty} \varphi_{\lambda_{\sigma(k)}} e_{\lambda_{\sigma(k)}}
$$

para toda permutação $\sigma: \mathbb{N} \rightarrow \mathbb{N}$. Então, por abuso de notação escrevemos

$$
\varphi=\sum_{\lambda \in \Gamma} \varphi_{\lambda} e_{\lambda}
$$

O conjunto $\mathcal{B}$ será chamado de base canônica de $c_{0}(\Gamma)$ ou $l_{p}(\Gamma), 1 \leq p<\infty$.

Proposição 1.4.1. Se $\Gamma$ é um conjunto infinito qualquer então $\left(c_{0}(\Gamma)\right)^{*}$ é isometricamente isomorfo a $l_{1}(\Gamma)$.

Demonstração. Seja $\mathcal{B}=\left\{e_{\lambda}: \lambda \in \Gamma\right\}$ a base canônica de $c_{0}(\Gamma)$. Verificaremos primeiramente que para cada $\varphi \in\left(c_{0}(\Gamma)\right)^{*}$ o conjunto $\Gamma_{\varphi}^{*}:=\left\{\lambda \in \Gamma: \varphi\left(e_{\lambda}\right) \neq 0\right\}$ é enumerável.

Com efeito, fixado $\varphi \in\left(c_{0}(\Gamma)\right)^{*}$ para cada $n \in \mathbb{N}$ definimos

$$
F_{n}:=\left\{\lambda \in \Gamma:\left|\varphi\left(e_{\lambda}\right)\right| \geq \frac{1}{n}\right\} .
$$

Se para algum $n_{0}$ o conjunto $F_{n_{0}}$ fosse infinito, poderíamos fixar um subconjunto infinito 
enumerável $\left\{\lambda_{k}: k \in \mathbb{N}\right\} \subseteq F_{n_{0}}$ e definir uma sequência $\left(v_{n}\right)_{n \in \mathbb{N}}$ onde

$$
v_{n}:=\sum_{k=1}^{n} \frac{1}{k} \frac{\overline{\varphi\left(e_{\lambda_{k}}\right)}}{\left|\varphi\left(e_{\lambda_{k}}\right)\right|} e_{\lambda_{k}} .
$$

Claramente $\left\|v_{n}\right\|_{\infty}=1, n \in \mathbb{N}$, e

$$
\begin{aligned}
\left|\varphi\left(v_{n}\right)\right| & =\left|\varphi\left(\sum_{k=1}^{n} \frac{1}{k} \frac{\overline{\varphi\left(e_{\lambda_{k}}\right)}}{\left|\varphi\left(e_{\lambda_{k}}\right)\right|} e_{\lambda_{k}}\right)\right| \\
& =\sum_{k=1}^{n} \frac{1}{k}\left|\varphi\left(e_{\lambda_{k}}\right)\right| \\
& \geq \frac{1}{n_{0}}\left(\sum_{k=1}^{n} \frac{1}{k}\right) .
\end{aligned}
$$

Isso implica uma contradição pelo fato de $\varphi$ ser contínua. Então $F_{n}$ é finito para todo $n \in \mathbb{N}$, e portanto, o conjunto

$$
\Gamma_{\varphi}^{*}=\bigcup_{n \in \mathbb{N}} F_{n}
$$

é enumerável.

Para cada $\varphi \in\left(c_{0}(\Gamma)\right)^{*}$, supondo sem perda de generalidade $\Gamma_{\varphi}^{*}=\left\{\lambda_{k}: k \in \mathbb{N}\right\}$, definimos para cada $n \in \mathbb{N}$

$$
u_{n}:=\sum_{k=1}^{n} \frac{\overline{\varphi\left(e_{\lambda_{k}}\right)}}{\left|\varphi\left(e_{\lambda_{k}}\right)\right|} e_{\lambda_{k}} .
$$

Claramente $\left\|u_{n}\right\|_{\infty}=1$ para cada $n \in \mathbb{N}$, e vale a seguinte relação

$$
\sum_{k=1}^{n}\left|\varphi\left(e_{\lambda_{k}}\right)\right|=\left|\varphi\left(\sum_{k=1}^{n} \frac{\overline{\varphi\left(e_{\lambda_{k}}\right)}}{\mid \varphi\left(e_{\lambda_{k}}\right)} e_{\lambda_{k}}\right)\right|=\left|\varphi\left(u_{n}\right)\right| \leq\|\varphi\|_{\infty} .
$$

Segue-se que

$$
\sum_{k=1}^{\infty}\left|\varphi\left(e_{\lambda_{k}}\right)\right| \leq\|\varphi\|_{\infty}
$$

e a convergência é claramente incondicional. Por abuso de notação escreveremos

$$
\sum_{\lambda \in \Gamma}\left|\varphi\left(e_{\lambda}\right)\right|:=\sum_{k=1}^{\infty}\left|\varphi\left(e_{\lambda_{k}}\right)\right| \leq\|\varphi\|_{\infty} .
$$


Podemos então, definir uma aplicação $\Phi:\left(c_{0}(\Gamma)\right)^{*} \rightarrow l_{1}(\Gamma)$, por

$$
\Phi(\varphi):=\left(\varphi\left(e_{\lambda}\right)\right)_{\lambda \in \Gamma}
$$

Verifica-se que $\Phi$ é uma aplicação linear, e da relação (1.1) segue-se imediatamente, $\|\Phi(\varphi)\|_{1} \leq\|\varphi\|_{\infty}$ para todo $\varphi \in\left(c_{0}(\Gamma)\right)^{*}$.

Observemos agora que para cada $\varphi \in\left(c_{0}(\Gamma)\right)^{*}$ e para cada $x=\left(x_{\lambda}\right)_{\lambda \in \Gamma}=\sum_{\lambda \in \Gamma} x_{\lambda} e_{\lambda} \in$ $B_{c_{0}(\Gamma)}$, vale a seguinte relação

$$
\begin{aligned}
|\varphi(x)| & =\left|\sum_{\lambda \in \Gamma} x_{\lambda} \varphi\left(e_{\lambda}\right)\right| \leq \sum_{\lambda \in \Gamma}\left|x_{\lambda}\right|\left|\varphi\left(e_{\lambda}\right)\right| \\
& \leq\left(\sum_{\lambda \in \Gamma}\left|\varphi\left(e_{\lambda}\right)\right|\right)=\|\Phi(\varphi)\|_{1},
\end{aligned}
$$

de onde concluímos, $\|\varphi\|_{\infty} \leq\|\Phi(\varphi)\|_{1}$ para todo $\varphi \in\left(c_{0}(\Gamma)\right)^{*}$.

Então $\Phi$ é uma isometria. Para verificarmos a sobrejetividade basta notar que para cada $\left(x_{\lambda}\right)_{\lambda \in \Gamma} \in l_{1}(\Gamma)$, a aplicação $\phi: c_{0}(\Gamma) \rightarrow \mathbb{K}$ definida por

$$
\phi(u):=\sum_{\lambda \in \Gamma} u_{\lambda} x_{\lambda}, u=\left(u_{\lambda}\right)_{\lambda \in \Gamma} \in c_{0}(\Gamma)
$$

é linear e contínua. Claramente $\Phi(\phi)=\left(x_{\lambda}\right)_{\lambda \in \Gamma}$.

De forma semelhante, demonstra-se que se $\frac{1}{p}+\frac{1}{q}=1$ então $\left(l_{p}(\Gamma)\right)^{*}$ é isometricamente isomorfo a $l_{q}(\Gamma)$ e que $\left(l_{1}(\Gamma)\right)^{*}$ é isometricamente isomorfo a $l_{\infty}(\Gamma)$.

A demonstração da proposição a seguir pode ser encontrada em [10].

\section{Proposição 1.4.2.}

(i) $c_{0}$ e $l_{p}, 1 \leq p<\infty$ são separáveis.

(ii) $l_{\infty}$ não é separável.

(iii) Se $\Gamma$ for um conjunto não enumerável, então $c_{0}(\Gamma)$ e $l_{p}(\Gamma), 1 \leq p \leq \infty$, não são separáveis.

Uma propriedade importante dos espaços $l_{1}(\Gamma)$ é que, sobre esse espaços, uma sequência converge fracamente se e somente se converge em norma. Tal propriedade é denominada propriedade de Schur. 
Definição 1.4.3. Dizemos que um espaço normado $X$ tem a propriedade de Schur, se toda sequência $\left(x_{n}\right)_{n \in \mathbb{N}}$ em $X$ convergindo fracamente a algum $x \in X$, converge em norma a $x$.

A proposição a seguir pode ser encontrada em [8].

Proposição 1.4.4. $l_{1}$ tem a propriedade de Schur.

Demonstração. Seja $\left(x_{n}\right)_{n \in \mathbb{N}} \subset l_{1}$ uma sequência arbitrária convergindo fracamente a algum $x \in l_{1}$. Dado $\epsilon>0$ arbitrário, definimos para cada $m \in \mathbb{N}$,

$$
\mathcal{B}_{m}:=\left\{\phi \in B_{l_{\infty}}:\left|\phi\left(x_{n}-x\right)\right| \leq \frac{\epsilon}{3}, n \geq m\right\} .
$$

Notando-se que para cada $n \in \mathbb{N}$, a aplicação $\beta_{n}: l_{\infty} \rightarrow \mathbb{K}$, definida por

$$
\beta_{n}(\phi):=\phi\left(x_{n}-x\right),
$$

é contínua, com respeito à topologia fraca* de $l_{\infty}$ e a topologia usual de $\mathbb{K}$, e

$$
\mathcal{B}_{m}=\left(\bigcap_{n \geq m} \beta_{n}^{-1}\left(B_{\frac{\epsilon}{3}}(0)\right)\right) \cap B_{l_{\infty}},
$$

concluímos que $\mathcal{B}_{m}$ é $w^{*}$-fechado para cada $m \in \mathbb{N}$. Por $\left(x_{n}\right)_{n \in \mathbb{N}}$ convergir fracamente a $x$, segue-se

$$
B_{l_{\infty}}=\bigcup_{m \in \mathbb{N}} \mathcal{B}_{m}
$$

Por $l_{1}^{*} \cong l_{\infty}$, o teorema de Alaoglu 1.2.16 implica que $B_{l_{\infty}}$ é $w^{*}$-compacto. Por $l_{1}$ ser separável, decorre da proposição 1.2.18, que $B_{l_{\infty}}$ é $w^{*}$-metrizável. Portanto, sob a topologia fraca* $B_{l_{\infty}}$ é um espaço métrico compacto e consequentemente, um espaço de Baire. Então, de acordo com o teorema de Baire, existe $m_{0} \in \mathbb{N}$ tal que o interior, com respeito à topologia fraca* $^{*}$, de $\mathcal{B}_{m_{0}}$ não seja vazio. Segue-se que existem $\phi_{0} \in B_{l_{\infty}}, y_{1}, \ldots, y_{r} \in l_{1}$ e $\delta_{0}>0$, tais que

$$
W\left(\phi_{0}, y_{1}, \ldots, y_{r}, \delta_{0}\right) \cap B_{l_{\infty}} \subset \mathcal{B}_{m_{0}} .
$$

Supondo $y_{i}=\left(y_{k}^{i}\right)_{k \in \mathbb{N}}, 1 \leq i \leq r$, fixemos $s \in \mathbb{N}$ tal que

$$
\sum_{k=s+1}^{\infty}\left|y_{k}^{i}\right|<\frac{\delta_{0}}{4}, 1 \leq i \leq r
$$

Se $\left\{e_{k}: k \in \mathbb{N}\right\}$ é a base canônica de $l_{1}$, para cada $\phi \in B_{l_{\infty}}$ satisfazendo

$$
\left|\phi\left(e_{i}\right)-\phi_{0}\left(e_{i}\right)\right| \leq \delta_{1}:=\frac{\delta_{0}}{2\left(\max _{1 \leq k \leq r}\left\|y_{k}\right\|_{1}+1\right)}, 1 \leq i \leq s,
$$


temos

$$
\begin{aligned}
\left|\phi\left(y_{i}\right)-\phi_{0}\left(y_{i}\right)\right| & =\left|\sum_{k=1}^{\infty}\left(\phi\left(e_{i}\right)-\phi_{0}\left(e_{i}\right)\right) y_{k}^{i}\right| \\
& \leq \sum_{k=1}^{s}\left|\phi\left(e_{i}\right)-\phi_{0}\left(e_{i}\right)\right|\left|y_{k}^{i}\right|+\sum_{k=s+1}^{\infty}\left(\|\phi\|+\left\|\phi_{0}\right\|\right)\left|y_{k}^{i}\right| \\
& \leq \delta_{1} \sum_{k=1}^{s}\left|y_{k}^{i}\right|+2 \frac{\delta_{0}}{4} \\
& \leq \delta_{1} \max _{1 \leq k \leq r}\left\|y_{k}\right\|_{1}+\frac{\delta_{0}}{2}<\delta_{0},
\end{aligned}
$$

ou seja, $\phi \in W\left(\phi_{0}, y_{1}, \ldots, y_{r}, \delta_{0}\right)$ e podemos concluir

$$
W\left(\phi_{0}, e_{1}, \ldots, e_{s}, \delta_{0}\right) \cap B_{l_{\infty}} \subset W\left(\phi_{0}, y_{1}, \ldots, y_{r}, \delta_{0}\right) \cap B_{l_{\infty}}
$$

Supondo para cada $n \in \mathbb{N}, x_{n}:=\left(\chi_{k}^{n}\right)_{k \in \mathbb{N}}$ e $x:=\left(\chi_{k}\right)_{k \in \mathbb{N}}$, temos

$$
\begin{aligned}
\left\|x_{n}-x\right\|_{1} & =\sum_{k=1}^{\infty}\left|\chi_{k}^{n}-\chi_{k}\right| \\
& =\sum_{k=1}^{s}\left|\chi_{k}^{n}-\chi_{k}\right|+\sum_{k=s+1}^{\infty}\left|\chi_{k}^{n}-\chi_{k}\right| \\
& =\sum_{k=1}^{s}\left|\chi_{k}^{n}-\chi_{k}\right|-\sum_{k=1}^{s} \phi_{0}\left(e_{k}\right)\left(\chi_{k}^{n}-\chi_{k}\right) \\
& +\sum_{k=1}^{s} \phi_{0}\left(e_{k}\right)\left(\chi_{k}^{n}-\chi_{k}\right)+\sum_{k=s+1}^{\infty}\left|\chi_{k}^{n}-\chi_{k}\right| \\
& \leq 2 \sum_{k=1}^{s}\left|\chi_{k}^{n}-\chi_{k}\right|+\left|\psi\left(x_{n}-x\right)\right|
\end{aligned}
$$

onde $\psi:=\left(\phi_{0}\left(e_{1}\right), \ldots, \phi_{0}\left(e_{s}\right), \operatorname{sng}\left(\chi_{s+1}^{n}-\chi_{s+1}^{n}\right), \operatorname{sng}\left(\chi_{s+2}^{n}-\chi_{s+2}^{n}\right), \ldots\right) \in B_{l_{\infty}} \mathrm{e}$

$$
\operatorname{sng}(u):= \begin{cases}\frac{\bar{u}}{|u|} & \text { se } u \neq 0 \\ 0 & \text { se } u=0\end{cases}
$$

Notando-se que $\left(\psi-\phi_{0}\right)\left(e_{k}\right)=0$ para todo $1 \leq k \leq s$, decorre

$$
\psi \in W\left(\phi_{0}, e_{1}, \ldots, e_{s}, \delta_{0}\right) \cap B_{l_{\infty}} \subset \mathcal{B}_{m_{0}} \subset \mathcal{B}_{n}, n \geq m_{0}
$$

Consequentemente $\left|\psi\left(x_{n}-x\right)\right| \leq \frac{\epsilon}{3}$ para todo $n \geq m_{0}$. 
Por $x_{n} \stackrel{w}{\rightarrow} x$, segue-se $\chi_{k}^{n} \rightarrow \chi_{k}, k \in \mathbb{N}$. Podemos fixar $m_{1} \in \mathbb{N}$ tal que

$$
\sum_{k=1}^{s}\left|\chi_{k}^{n}-\chi_{k}\right|<\frac{\epsilon}{3}, n \geq m_{1}
$$

Então para todo $n>\max \left\{m_{0}, m_{1}\right\}$

$$
\left\|x_{n}-x\right\|_{1}<2 \frac{\epsilon}{3}+\frac{\epsilon}{3}=\epsilon
$$

e isso demonstra que $x_{n} \rightarrow x$ em $l_{1}$.

Proposição 1.4.5. Para qualquer conjunto $\Gamma$ não enumerável, $l_{1}(\Gamma)$ tem a propriedade de Schur

Demonstração. Seja $\left(x_{n}\right)_{n \in \mathbb{N}}$ uma sequência em $l_{1}(\Gamma)$, convergindo fracamente a algum $x \in$ $l_{1}(\Gamma)$.

Para cada $y=\left(y_{\lambda}\right)_{\lambda \in \Gamma} \in l_{1}(\Gamma)$, de acordo com a definição de $l_{1}(\Gamma)$, o conjunto $\Gamma_{y}:=$ $\left\{\lambda \in \Gamma: y_{\lambda} \neq 0\right\}$ é enumerável. Seja

$$
\Gamma_{0}:=\left(\bigcup_{n \in \mathbb{N}} \Gamma_{x_{n}}\right) \cup \Gamma_{x} .
$$

Claramente $\Gamma_{0}$ é enumerável e podemos, sem perda de generalidade, supor

$$
\Gamma_{0}=\left\{\lambda_{k}: k \in \mathbb{N}\right\}
$$

Observemos que para cada $y=\left(y_{\lambda}\right)_{\lambda \in \Gamma} \in l_{1}(\Gamma)$, vale a seguinte relação

$$
\sum_{k \in \mathbb{N}}\left|y_{\lambda_{k}}\right| \leq \sum_{\lambda \in \Gamma}\left|y_{\lambda}\right|=\left\|\left(y_{\lambda}\right)_{\lambda \in \Gamma}\right\|_{1}
$$

Podemos então definir uma aplicação $\Phi: l_{1}(\Gamma) \rightarrow l_{1}$ por

$$
\Phi\left(\left(y_{\lambda}\right)_{\lambda \in \Gamma}\right):=\left(y_{\lambda_{k}}\right)_{k \in \mathbb{N}}
$$

Sem dificuldade, demonstra-se que $\Phi$ é linear, e da relação (1.3), segue-se imediatamente que $\Phi$ é contínua.

Em virtude da proposição 1.2 .5 , a sequência $\left(\Phi\left(x_{n}\right)\right)_{n \in \mathbb{N}}$ em $l_{1}$ converge fracamente a $\Phi(x)$. 
Por $l_{1}$ ter a propriedade de Schur, $\left(\Phi\left(x_{n}\right)\right)_{n \in \mathbb{N}}$ converge em norma para $\Phi(x)$ e de acordo com a definição de $\Phi$, temos

$$
\left\|\Phi\left(x_{n}\right)-\Phi(x)\right\|_{1}=\left\|\Phi\left(x_{n}-x\right)\right\|_{1}=\left\|x_{n}-x\right\|_{1}, n \in \mathbb{N} .
$$

Concluímos que a sequência $\left(x_{n}\right)_{n \in \mathbb{N}}$ converge para $x$ em norma. 


\section{Capítulo 2}

\section{Aplicações Multilineares e Polinômios}

Este capítulo é dedicado ao estudo de conceitos básicos sobre aplicações multilineares, polinômios e tópicos relacionados. Para um estudo mais detalhado, recomendamos [9] e [17].

\subsection{Aplicações Multilineares}

Definição 2.1.1. Sejam $X_{1}, \ldots, X_{n}$ e $Y$, espaços vetoriais sobre um corpo $\mathbb{K}$. Dizemos que uma aplicação $A: X_{1} \times \ldots \times X_{n} \rightarrow Y$ é $n$-linear se for linear em cada variável separadamente, ou seja

$$
A\left(x_{1}, \ldots, x_{i}+\lambda y_{i}, \ldots, x_{n}\right)=A\left(x_{1} \ldots, x_{i}, \ldots, x_{n}\right)+\lambda A\left(x_{1}, \ldots, y_{i}, \ldots, x_{n}\right)
$$

para quaisquer $x_{1}, \ldots, x_{i}, y_{i}, \ldots, x_{n} \in X_{i}, 1 \leq i \leq n$ e $\lambda \in \mathbb{K}$.

Verifica-se facilmente que o conjunto de todas as aplicações $n$-lineares de $X_{1}, \ldots, X_{n}$ em $Y$, sob as operações usuais de adição e multiplicação por escalar, é um espaço vetorial. Este espaço será denotado por $\mathcal{L}_{a}\left(X_{1}, \ldots, X_{n} ; Y\right)$. Em particular, se $X_{1}=\ldots=X_{n}=$ $X$ denotaremos $\mathcal{L}_{a}\left(X_{1}, \ldots, X_{n} ; Y\right):=\mathcal{L}_{a}\left({ }^{n} X ; Y\right), \mathcal{L}_{a}\left({ }^{1} X ; Y\right)=\mathcal{L}_{a}(X ; Y)$ e por convenção $\mathcal{L}_{a}\left({ }^{0} X ; Y\right)=Y$. Se $Y=\mathbb{K}$, denotaremos $\mathcal{L}_{a}\left(X_{1}, \ldots, X_{n} ; \mathbb{K}\right):=\mathcal{L}_{a}\left(X_{1}, \ldots, X_{n}\right)$.

A partir de agora, menos que sejam mencionadas outras hipóteses, $X_{1}, \ldots, X_{n}, X, Y$ denotarão espaços normados. Por questão de simplicidade e quando não houver possibilidade de confusão, qualquer norma será denotada apenas por $\|$.$\| , ficando claro pelo contexto a$ qual espaço se refere.

A topologia produto sobre $X_{1} \times \ldots \times X_{n}$ pode ser gerada pela norma

$$
\left\|\left(x_{1}, \ldots, x_{n}\right)\right\|:=\max _{1 \leq k \leq n}\left\|x_{k}\right\| .
$$


Neste trabalho, sobre um produto de espaços normados, esta será a norma utilizada.

Demonstra-se sem dificuladade que $X_{1} \times \ldots \times X_{n}$ é um espaço de Banach se e somente se $X_{1}, \ldots, X_{n}$ são espaços de Banach.

Proposição 2.1.2. Para cada $A \in \mathcal{L}_{a}\left(X_{1}, \ldots, X_{n} ; Y\right)$ são equivalentes:

(i) A é contínua;

(ii) A é contínua na origem;

(iii) Existe $M>0$ tal que $\left\|A\left(x_{1}, \ldots, x_{n}\right)\right\| \leq M\left\|x_{1}\right\| \ldots\left\|x_{n}\right\|$ para todo $\left(x_{1}, \ldots, x_{n}\right) \in$ $X_{1} \times \ldots \times X_{n}$.

Demonstração. $(i) \Rightarrow($ ii $)$ Evidente.

$($ ii $) \Rightarrow($ iii $)$ Se $A$ é contínua na origem, então existe $\delta>0$ tal que $\left\|A\left(x_{1}, \ldots, x_{n}\right)\right\| \leq 1$ sempre que $\left\|\left(x_{1}, \ldots, x_{n}\right)\right\| \leq \delta$.

Fixando-se $M:=\frac{1}{\delta^{n}}$, seja $\left(x_{1}, \ldots, x_{n}\right) \in X_{1} \times \ldots \times X_{n}$ arbitrário. Se $x_{k}=0$ para algum $1 \leq k \leq n$ então

$$
\left\|A\left(x_{1}, \ldots, x_{n}\right)\right\|=0=M\left\|x_{1}\right\| \ldots\left\|x_{n}\right\| .
$$

Se $x_{k} \neq 0,1 \leq k \leq n$, então

$$
\left\|A\left(\frac{\delta x_{1}}{\left\|x_{1}\right\|}, \ldots, \frac{\delta x_{n}}{\left\|x_{n}\right\|}\right)\right\| \leq 1
$$

pois claramente

$$
\left\|\left(\frac{\delta x_{1}}{\left\|x_{1}\right\|}, \ldots, \frac{\delta x_{n}}{\left\|x_{n}\right\|}\right)\right\| \leq \delta .
$$

Consequentemente

$$
\left\|A\left(x_{1}, \ldots, x_{n}\right)\right\| \leq \frac{1}{\delta^{n}}\left\|x_{1}\right\| \ldots\left\|x_{n}\right\|=M\left\|x_{1}\right\| \ldots\left\|x_{n}\right\|
$$

$($ iii $) \Rightarrow(i)$ Fixado $m \in \mathbb{N}$ sejam $y=\left(y_{1}, \ldots, y_{n}\right)$ e $x=\left(x_{1}, \ldots, x_{n}\right) \in \mathcal{B}_{m}(0)$, onde $\mathcal{B}_{m}(0):=$ $\{x \in X:\|x\| \leq m\}$. 
Definindo-se $z_{0}:=x, z_{1}:=\left(y_{1}, x_{2} \ldots, x_{n}\right), z_{2}:=\left(y_{1}, y_{2}, x_{3} \ldots, x_{n}\right), \ldots, z_{n}:=y$, temos

$$
\begin{aligned}
\|A(y)-A(x)\| & =\left\|\sum_{k=1}^{n}\left(A\left(z_{k}\right)-A\left(z_{k-1}\right)\right)\right\| \leq \sum_{k=1}^{n}\left\|A\left(z_{k}\right)-A\left(z_{k-1}\right)\right\| \\
& =\sum_{k=1}^{n}\left\|A\left(y_{1}, \ldots, y_{k}-x_{k}, \ldots, x_{n}\right)\right\| \\
& \leq \sum_{k=1}^{n} M\left\|x_{1}\right\| \ldots\left\|y_{k}-x_{k}\right\| \ldots\left\|x_{n}\right\| \\
& \leq M m^{n-1}\left(\sum_{k=1}^{n}\left\|y_{k}-x_{k}\right\|\right) .
\end{aligned}
$$

A relação acima implica claramente a continuidade de $A$.

Denotaremos por $\mathcal{L}\left(X_{1}, \ldots, X_{n} ; Y\right)$ o subespaço de $\mathcal{L}_{a}\left(X_{1}, \ldots, X_{n} ; Y\right)$ consistindo de todas as aplicações $n$-lineares contínuas. Em particular, se $X_{1}=\ldots=X_{n}=X$ denotaremos $\mathcal{L}\left({ }^{n} X ; Y\right):=\mathcal{L}\left(X_{1}, \ldots, X_{n} ; Y\right)$. Denotaremos também $\mathcal{L}\left(X_{1}, \ldots, X_{n}\right):=\mathcal{L}\left(X_{1}, \ldots, X_{n} ; \mathbb{K}\right)$ e $\mathcal{L}\left({ }^{n} X\right):=\mathcal{L}\left({ }^{n} X ; \mathbb{K}\right)$.

A seguir um exemplo de aplicação multilinear não contínua.

Exemplo 2.1.3. Seja $X=\left(l_{1},\|\cdot\|_{\infty}\right)$ e $A \in \mathcal{L}_{a}\left({ }^{2} X\right)$ definida por

$$
A\left(\left(\alpha_{k}\right)_{k \in \mathbb{N}},\left(\beta_{k}\right)_{k \in \mathbb{N}}\right):=\sum_{k=1}^{\infty} \alpha_{k} \beta_{k}, \quad\left(\alpha_{k}\right)_{k \in \mathbb{N}},\left(\beta_{k}\right)_{k \in \mathbb{N}} \in l_{1} .
$$

Para cada $n \in \mathbb{N}$, seja $x_{n}=(\overbrace{1, \ldots, 1}^{n}, 0, \ldots)$. Claramente $\left\|\left(x_{n}, x_{n}\right)\right\|=1$ para todo $n \in \mathbb{N}$ e $\left\|A\left(x_{n}, x_{n}\right)\right\|=n$. De acordo com a proposição 2.1.2, $A$ não é contínua.

Observamos no entanto que, para cada $\left(\alpha_{k}\right)_{k \in \mathbb{N}},\left(\beta_{k}\right)_{k \in \mathbb{N}} \in l_{1}$, são válidas as seguintes relações:

$$
\begin{aligned}
& \left\|A\left(\left(\alpha_{k}\right)_{k \in \mathbb{N}},\left(\beta_{k}\right)_{k \in \mathbb{N}}\right)\right\|=\left\|\sum_{k}^{\infty} \alpha_{k} \beta_{k}\right\| \leq\left\|\left(\alpha_{k}\right)_{k \in \mathbb{N}}\right\|_{1}\left\|\left(\beta_{k}\right)_{k \in \mathbb{N}}\right\|_{\infty}, \\
& \left\|A\left(\left(\alpha_{k}\right)_{k \in \mathbb{N}},\left(\beta_{k}\right)_{k \in \mathbb{N}}\right)\right\|=\left\|\sum_{k}^{\infty} \alpha_{k} \beta_{k}\right\| \leq\left\|\left(\beta_{k}\right)_{k \in \mathbb{N}}\right\|_{1}\left\|\left(\alpha_{k}\right)_{k \in \mathbb{N}}\right\|_{\infty} .
\end{aligned}
$$

Essas relações implicam a continuidade de $A$ em cada variável separadamente, ou seja, fixando-se qualquer uma das variáveis, obtém-se uma transformação linear contínua. 
Devemos observar também que o espaço $\left(l_{1},\|\cdot\|_{\infty}\right)$ não é de Banach. A proposição a seguir nos mostra que se os espaços forem de Banach, em uma aplicação multilinear, a continuidade em cada variável separadamente implica a continuidade.

Proposição 2.1.4. Sejam $X_{1}, \ldots, X_{n}$ espaços de Banach e $Y$ um espaço normado. Uma aplicação $A \in \mathcal{L}_{a}\left(X_{1}, \ldots, X_{n} ; Y\right)$ é contínua se e somente se é contínua em cada variável separadamente.

Demonstração. Claramente, se $A$ for contínua então é contínua em cada variável separadamente.

A demonstração da afirmação recíproca será por indução sobre $n$. Se $n=1$, a tese segue trivialmente. Supondo $n>1$ e a tese válida em $\mathcal{L}_{a}\left(X_{1}, \ldots, X_{n-1} ; Y\right)$, fixemos uma sequência $\left(\left(x_{k}, y_{k}\right)\right)_{k \in \mathbb{N}}$ em $X_{1} \times \ldots \times X_{n}$, com $x_{k} \in X_{1} \times \ldots \times X_{n-1}$ e $y_{k} \in X_{n}$, convergindo a zero. Seja $\mathcal{F}=\left\{A_{k}: k \in \mathbb{N}\right\} \subset \mathcal{L}_{a}\left(X_{n} ; Y\right)$ onde

$$
A_{k}(y):=A\left(x_{k}, y\right), k \in \mathbb{N} .
$$

Para cada $y \in X_{n}$ a aplicação $A_{y}: X_{1} \times \ldots \times X_{n-1} \rightarrow Y$ definida por

$$
A_{y}(x):=A(x, y)
$$

é $n$-1-linear e contínua em cada variável separadamente. De acordo com a hipótese de indução, $A_{y}$ é contínua. Então para cada $k \in \mathbb{N}$ e $y \in X_{n}$ temos

$$
\lim _{k \rightarrow \infty} A_{k}(y)=\lim _{k \rightarrow \infty} A\left(x_{k}, y\right)=\lim _{k \rightarrow \infty} A_{y}\left(x_{k}\right)=0
$$

Consequentemente, $\mathcal{F}$ é uma coleção de aplicações lineares pontualmente limitada. Pelo Princípio da Limitação Uniforme, existe $M>0$ tal que $\sup _{k \in \mathbb{N}}\left\|A_{k}\right\| \leq M$. Então

$$
\lim _{k \rightarrow \infty}\left\|A\left(x_{k}, y_{k}\right)\right\|=\lim _{k \rightarrow \infty}\left\|A_{k} y_{k}\right\| \leq \lim _{k \rightarrow \infty} M\left\|y_{k}\right\|=0
$$

Concluímos que $A$ é contínua na origem e portanto, em virtude da proposição 2.1.2, $A$ é contínua.

Proposição 2.1.5. A aplicação $\|\|:. \mathcal{L}\left(X_{1}, \ldots, X_{n} ; Y\right) \rightarrow \mathbb{R}$ definida por

$$
\|A\|:=\sup \left\{\|A(x)\|: x \in X_{1} \times \ldots \times X_{n},\|x\| \leq 1\right\}
$$

define uma norma sobre $\mathcal{L}\left(X_{1}, \ldots, X_{n} ; Y\right)$. 
Demonstração. Segue da proposição 2.1.2 que para todo $A \in \mathcal{L}\left({ }^{n} X ; Y\right),\|A\|<\infty$ e

$$
\left\|A\left(x_{1}, \ldots, x_{n}\right)\right\| \leq\|A\|\left\|x_{1}\right\| \ldots\left\|x_{n}\right\| .
$$

Claramente, para cada $A \in \mathcal{L}\left({ }^{n} X ; Y\right)$, temos $\|A\| \geq 0$. Se $\|A\|=0$, da relação (2.1), temos para cada $\left(x_{1}, \ldots, x_{n}\right)$

$$
\left\|A\left(x_{1}, \ldots, x_{n}\right)\right\| \leq\|A\|\left\|x_{1}\right\| \ldots\left\|x_{n}\right\|=0
$$

portanto $A \equiv 0$.

Fixado $\lambda \in \mathbb{K}$ e $\left\|\left(x_{1}, \ldots, x_{n}\right)\right\| \leq 1$ temos

$$
\left\|\lambda A\left(x_{1}, \ldots, x_{n}\right)\right\|=|\lambda|\left\|A\left(x_{1}, \ldots, x_{n}\right)\right\| \leq|\lambda|\|A\|,
$$

consequentemente

$$
\|\lambda A\| \leq|\lambda|\|A\| .
$$

Se $\lambda=0$, claramente vale a igualdade na relação (2.2). Se $\lambda \neq 0$ temos

$$
\left\|A\left(x_{1}, \ldots, x_{n}\right)\right\|=\left\|\lambda\left(\frac{1}{\lambda}\right) A\left(x_{1}, \ldots, x_{n}\right)\right\|=\frac{1}{|\lambda|}\left\|\lambda A\left(x_{1}, \ldots, x_{n}\right)\right\| \leq \frac{1}{|\lambda|}\|\lambda A\|,
$$

portanto

$$
|\lambda|\|A\| \leq\|\lambda A\| .
$$

Das relações (2.2) e (2.3) decorre a igualdade para toda aplicação $A \in \mathcal{L}\left(X_{1}, \ldots, X_{n} ; Y\right)$. Se $A, B \in \mathcal{L}\left(X_{1}, \ldots, X_{n} ; Y\right)$, fixado $\left\|\left(x_{1}, \ldots, x_{n}\right)\right\| \leq 1$ arbitrário temos

$$
\left\|A\left(x_{1}, \ldots, x_{n}\right)+B\left(x_{1}, \ldots, x_{n}\right)\right\| \leq\left\|A\left(x_{1}, \ldots, x_{n}\right)\right\|+\left\|B\left(x_{1}, \ldots, x_{n}\right)\right\| \leq\|A\|+\|B\|,
$$

portanto

$$
\|A+B\| \leq\|A\|+\|B\|
$$

Proposição 2.1.6. Se $Y$ é um espaço de Banach então, sob a norma $A \mapsto\|A\|$, o espaço $\mathcal{L}\left(X_{1}, \ldots, X_{n} ; Y\right)$ é de Banach.

Demonstração. Seja $\left(A_{k}\right)_{k \in \mathbb{N}}$ uma sequencia de Cauchy em $\mathcal{L}\left(X_{1}, \ldots, X_{n} ; Y\right)$. Para cada $\left(x_{1}, \ldots, x_{n}\right) \in X_{1} \times \ldots \times X_{n}$ e para cada $k, r \in \mathbb{N}$,

$$
\left\|A_{k}\left(x_{1}, \ldots, x_{n}\right)-A_{r}\left(x_{1}, \ldots, x_{n}\right)\right\| \leq\left\|A_{k}-A_{r}\right\|\left\|x_{1}\right\| \ldots\left\|x_{n}\right\| .
$$


Então, para cada $u \in X_{1} \times \ldots \times X_{n}$, a sequência $\left(A_{k}(u)\right)_{k \in \mathbb{N}}$ é de Cauchy em $Y$ e converge, pois $Y$ é completo.

Podemos então, definir uma aplicação $A: X_{1} \times \ldots \times X_{n} \rightarrow Y$ por

$$
A(u):=\lim _{k \rightarrow \infty} A_{k}(u)
$$

A $n$-linearidade da aplicação $A$ segue das propriedades operatórias do limite de sequências e da $n$-linearidade de cada uma das aplicações $A_{k}$.

Para verificarmos a continuidade de $A$, fixemos $u \in X_{1} \times \ldots \times X_{n}$ com $\|u\| \leq 1$ arbitrário. De acordo com a relação $(2.1)$, deve existir $k_{0} \in \mathbb{N}$ tal que

$$
\left\|A_{k}(u)-A_{r}(u)\right\| \leq 1
$$

sempre que $k, r \geq k_{0}$. Então, para todo $k \geq k_{0}$, a desigualdade triangular implica

$$
\left\|A_{k}(u)\right\| \leq\left\|A_{k_{0}}(u)\right\|+1 \leq\left\|A_{k_{0}}\right\|+1
$$

e consequentemente

$$
\|A\| \leq\left\|A_{k_{0}}\right\|+1
$$

Concluímos que $\|A\| \leq \infty$, e em virtude da proposição 2.1.2, $A$ é contínua.

Finalmente, fixando-se $\epsilon>0$, existe $k_{1} \in \mathbb{N}$ tal que para quaisquer $k, r \geq k_{1}$

$$
\left\|A_{k}-A_{r}\right\| \leq \frac{\epsilon}{2}
$$

Seja $\|u\| \leq 1$ arbitrário. Para quaisquer $k, r \geq k_{1}$

$$
\left\|A_{k}(u)-A_{r}(u)\right\| \leq\left\|A_{k}-A_{r}\right\| \leq \frac{\epsilon}{2}
$$

Fixando-se $k \geq k_{1}$ temos

$$
\lim _{r \rightarrow \infty}\left\|A_{k}(u)-A_{r}(u)\right\|=\left\|A_{k}(u)-A(u)\right\| \leq \frac{\epsilon}{2}<\epsilon .
$$

Por $\|u\| \leq 1$ ser arbitrário,

$$
\left\|A_{k}-A\right\|<\epsilon
$$

sempre que $k \geq k_{0}$. Concluímos que $\left(A_{k}\right)_{k \in \mathbb{N}}$ converge para $A$. 


\subsection{Aplicações Multilineares Simétricas}

As aplicações multilineares simétricas têm importância fundamental no estudo de polinômios, objetos que estudaremos a partir da próxima seção. Denotaremos por $\mathcal{S}_{n}$ o conjunto de todas as permutações de $\{1, \ldots, n\}$, ou seja, o conjunto de todas as bijeções de $\{1, \ldots, n\}$ em $\{1, \ldots, n\}$.

Definição 2.2.1. Dizemos que $A \in \mathcal{L}_{a}\left({ }^{n} X ; Y\right)$ é simétrica se para quaisquer $x_{1}, \ldots, x_{n} \in X$ e para todo $\sigma \in \mathcal{S}_{n}$

$$
A\left(x_{1}, \ldots, x_{n}\right)=A\left(x_{\sigma(1)}, \ldots, x_{\sigma(n)}\right) .
$$

Verifica-se facilmente que o conjunto de todas as aplicações multilineares simétricas é um subespaço de $\mathcal{L}_{a}\left({ }^{n} X ; Y\right)$ e será denotado por $\mathcal{L}_{a}^{s}\left({ }^{n} X ; Y\right)$. Em particular, denotaremos $\mathcal{L}_{a}^{s}\left({ }^{n} X\right):=\mathcal{L}_{a}^{s}\left({ }^{n} X ; \mathbb{K}\right)$.

Definição 2.2.2. Para cada $\alpha=\left(\alpha_{1}, \ldots, \alpha_{m}\right) \in \mathbb{N}_{0}^{m}:=\overbrace{\mathbb{N}_{0} \times \ldots \times \mathbb{N}_{0}}^{m}$, definimos

$$
|\alpha|:=\alpha_{1}+\ldots+\alpha_{m} \quad \text { e } \quad \alpha !:=\alpha_{1} ! \ldots \alpha_{m} !
$$

Em diversos momentos neste trabalho, tendo em vista simplificar algumas notações, dado $A \in \mathcal{L}_{a}\left({ }^{n} X ; Y\right), x_{1}, \ldots, x_{m} \in X$ e $\alpha=\left(\alpha_{1}, \ldots, \alpha_{m}\right) \in \mathbb{N}_{0}^{m}$ com $|\alpha|=n$, denotaremos

$$
A x_{1}^{\alpha_{1}} \ldots x_{m}^{\alpha_{m}}:=A \underbrace{x_{1} \ldots x_{1}}_{\alpha_{1}} \ldots \underbrace{x_{m} \ldots x_{m}}_{\alpha_{m}} .
$$

Denotaremos por $\mathcal{L}^{s}\left({ }^{n} X ; Y\right)$ o subespaço de $\mathcal{L}_{a}^{s}\left({ }^{n} X ; Y\right)$ de todas as aplicações $n$-lineares simétricas contínuas. Em particular, denotaremos $\mathcal{L}^{s}\left({ }^{n} X\right):=\mathcal{L}^{s}\left({ }^{n} X ; \mathbb{K}\right)$.

Corolário 2.2.3. Se $Y$ é um espaço de Banach então $\mathcal{L}^{s}\left({ }^{n} X ; Y\right)$ é um espaço de Banach.

Demonstração. Segue diretamente da proposição 2.1.6 e da unicidade do limite de uma sequência em um espaço normado.

Proposição 2.2.4. Para cada $A \in \mathcal{L}\left({ }^{n} X ; Y\right)$ seja $A^{s}$ definida por

$$
A^{s}\left(x_{1}, \ldots, x_{n}\right):=\frac{1}{n !} \sum_{\sigma \in \mathcal{S}_{n}} A\left(x_{\sigma(1)}, \ldots, x_{\sigma(n)}\right), x_{1}, \ldots, x_{n} \in X .
$$


Então a aplicação $A \mapsto A^{s}$ é uma projeção de $\mathcal{L}\left({ }^{n} X ; Y\right)$ em $\mathcal{L}^{s}\left({ }^{n} X ; Y\right)$, e para cada $A \in \mathcal{L}\left({ }^{n} X ; Y\right)$,

$$
\|A\| \leq\left\|A^{s}\right\|
$$

Denominaremos $A^{s}$, a aplicação n-linear simétrica associada a $A$.

Demonstração. Fixado $A \in \mathcal{L}\left({ }^{n} X ; Y\right)$, claramente $A^{s}$ é uma aplicação multilinear contínua e para cada $\gamma \in \mathcal{S}_{n}$

$$
\begin{aligned}
A^{s}\left(x_{\gamma(1)}, \ldots, x_{\gamma(n)}\right) & =\frac{1}{n !} \sum_{\sigma \in \mathcal{S}_{n}} A\left(x_{\gamma(\sigma(1))}, \ldots, x_{\gamma(\sigma(n))}\right) \\
& =\frac{1}{n !} \sum_{\beta \in \mathcal{S}_{n}} A\left(x_{\beta(1)}, \ldots, x_{\beta(n)}\right)=A^{s}\left(x_{1}, \ldots, x_{n}\right) .
\end{aligned}
$$

Então, de fato $A^{s} \in \mathcal{L}^{s}\left({ }^{n} X ; Y\right)$. Claramente $A \mapsto A^{s}$ é uma aplicação linear e para cada $A \in \mathcal{L}\left({ }^{n} X ; Y\right)$

$$
\begin{aligned}
\left(A^{s}\right)^{s}\left(x_{1}, \ldots, x_{n}\right) & =\frac{1}{n !} \sum_{\sigma \in \mathcal{S}_{n}} A^{s}\left(x_{\sigma(1)}, \ldots, x_{\sigma(n)}\right) \\
& =\frac{1}{n !} \sum_{\sigma \in \mathcal{S}_{n}} A^{s}\left(x_{1}, \ldots, x_{n}\right)=A^{s}\left(x_{1}, \ldots, x_{n}\right) .
\end{aligned}
$$

Concluímos que a aplicação $A \mapsto A^{s}$ é uma projeção de $\mathcal{L}\left({ }^{n} X ; Y\right)$ em $\mathcal{L}^{s}\left({ }^{n} X ; Y\right)$.

Fixando-se $\left\|\left(x_{1}, \ldots, x_{n}\right)\right\| \leq 1$ arbitrário, temos

$$
\left\|A^{s}\left(x_{1}, \ldots, x_{n}\right)\right\|=\left\|\frac{1}{n !} \sum_{\sigma \in \mathcal{S}_{n}} A\left(x_{1}, \ldots, x_{n}\right)\right\| \leq \frac{1}{n !}\|A\| \sum_{\sigma \in \mathcal{S}_{n}} 1=\|A\| .
$$

Consequentemente $\left\|A^{s}\right\| \leq\|A\|$.

Mais geralmente a aplicação $A \mapsto A^{s}$, associando cada $A \in \mathcal{L}_{a}\left({ }^{n} X ; Y\right)$ a aplicação $A^{s} \in$ $\mathcal{L}_{a}^{s}\left({ }^{n} X ; Y\right)$, definida por

$$
A^{s}\left(x_{1}, \ldots, x_{n}\right):=\frac{1}{n !} \sum_{\sigma \in \mathcal{S}_{n}} A\left(x_{\sigma(1)}, \ldots, x_{\sigma(n)}\right), x_{1}, \ldots, x_{n} \in X
$$

é uma projeção de $\mathcal{L}_{a}\left({ }^{n} X ; Y\right)$ em $\mathcal{L}_{a}^{s}\left({ }^{n} X ; Y\right)$.

Analogamente ao caso contínuo, denominaremos $A^{s}$, a aplicação $n$-linear simétrica associada a $A$. 
Proposição 2.2.5. (Fórmula de Leibniz) Seja $A \in \mathcal{L}_{a}^{s}\left({ }^{n} X ; Y\right)$. Para $x_{1}, \ldots, x_{m} \in X$,

$$
A\left(x_{1}+\ldots+x_{m}\right)^{n}=\sum_{|\alpha|=n} \frac{n !}{\alpha !} A x_{1}^{\alpha_{1}} \ldots x_{m}^{\alpha_{m}},
$$

onde $\alpha=\left(\alpha_{1}, \ldots, \alpha_{m}\right) \in \mathbb{N}_{0}^{m}$, tal que $|\alpha|=n$.

Demonstração. A demonstração será feita por indução em $n$. É evidente que a proposição se verifica para $n=0$ e $n=1$. Se $n>1$, supondo válida a tese em $\mathcal{L}_{a}^{s}{ }^{\left({ }^{n-1} X ; Y\right) \text { fixemos }}$ $x_{1}, \ldots, x_{m} \in X$. Aplicando-se a hipótese de indução para a aplicação $B \in \mathcal{L}_{a}^{s}\left({ }^{n-1} X ; Y\right)$ definida por

$$
B\left(y_{1}, \ldots, y_{n-1}\right):=A\left(x_{1}+\ldots+x_{m}\right)\left(y_{1}, \ldots, y_{n-1}\right), y_{1}, \ldots, y_{n-1} \in X
$$

teremos

$$
\begin{aligned}
A\left(x_{1}+\ldots+x_{m}\right)^{n} & =B\left(x_{1}+\ldots+x_{m}\right)^{n-1} \\
& =\sum_{|\alpha|=n-1} \frac{(n-1) !}{\alpha !} B x_{1}^{\alpha_{1}} \ldots x_{m}^{\alpha_{m}} \\
& =\sum_{|\alpha|=n-1} \frac{(n-1) !}{\alpha !} A\left(x_{1}+\ldots+x_{m}\right) x_{1}^{\alpha_{1}} \ldots x_{m}^{\alpha_{m}} \\
& =\sum_{|\alpha|=n-1} \frac{(n-1) !}{\alpha !} A x_{1}^{\alpha_{1}+1} \ldots x_{m}^{\alpha_{m}}+\ldots+\sum_{|\alpha|=n-1} \frac{(n-1) !}{\alpha !} A x_{1}^{\alpha_{1}} \ldots x_{m}^{\alpha_{m}+1} .
\end{aligned}
$$

Para cada $1 \leq k \leq m$, definimos $\beta^{(k)}=\left(\beta_{1}^{(k)}, \ldots, \beta_{m}^{(k)}\right)$ onde $\beta_{1}^{(k)}:=\alpha_{1}, \ldots, \beta_{k}^{(k)}:=$ $\alpha_{k}+1, \ldots, \beta_{m}^{(k)}:=\alpha_{m}$. 
Podemos escrever

$$
\begin{aligned}
& \sum_{|\alpha|=n-1} \frac{(n-1) !}{\alpha !} A x_{1}^{\alpha_{1}+1} \ldots x_{m}^{\alpha_{m}}+\ldots+\sum_{|\alpha|=n-1} \frac{(n-1) !}{\alpha !} A x_{1}^{\alpha_{1}} \ldots x_{m}^{\alpha_{m}+1} \\
= & \sum_{\left|\beta^{(1)}\right|=n} \frac{\beta_{1}^{(1)}(n-1) !}{\beta \beta^{(1) !}} A x_{1}^{\beta_{1}^{(1)}} \ldots x_{m}^{\beta_{m}^{(1)}}+\ldots+\sum_{\left|\beta^{(m)}\right|=n} \frac{\beta_{m}^{(m)}(n-1) !}{\beta^{(m)} !} A x_{1}^{\beta_{1}^{(m)}} \ldots x_{m}^{\beta_{m}^{(m)}} \\
= & \sum_{|\beta|=n} \frac{\beta_{1}(n-1) !}{\beta !} A x_{1}^{\beta_{1}} \ldots x_{m}^{\beta_{m}}+\ldots+\sum_{|\beta|=n} \frac{\beta_{m}(n-1) !}{\beta !} A x_{1}^{\beta_{1}} \ldots x_{m}^{\beta_{m}} \\
= & \sum_{|\beta|=n} \frac{\left(\beta_{1}+\ldots+\beta_{m}\right)(n-1) !}{\beta !} A x_{1}^{\beta_{1}} \ldots x_{m}^{\beta_{m}} \\
= & \sum_{|\beta|=n} \frac{n !}{\beta !} A x_{1}^{\beta_{1}} \ldots x_{m}^{\beta_{m}}
\end{aligned}
$$

como queríamos.

Corolário 2.2.6. (Fórmula do Binômio) Seja $A \in \mathcal{L}_{a}^{s}\left({ }^{n} X ; Y\right)$. Para $x, y \in X$,

$$
A(x+y)^{n}=\sum_{k=0}^{n}\left(\begin{array}{l}
n \\
k
\end{array}\right) A x^{n-k} y^{k} .
$$

O próximo resultado estabelece que uma aplicação multilinear simétrica é determinada pelos seus valores na diagonal de $X^{n}$.

Proposição 2.2.7. (Fórmula de Polarização) $S e A \in \mathcal{L}_{a}^{s}\left({ }^{n} X ; Y\right)$, então para todo $x_{0}, \ldots, x_{n} \in X$

$$
A\left(x_{1}, \ldots, x_{n}\right)=\frac{1}{n ! 2^{n}} \sum_{\epsilon_{k}= \pm 1} \epsilon_{1} \ldots \epsilon_{n} A\left(x_{0}+\epsilon_{1} x_{1}+\ldots+\epsilon_{n} x_{n}\right)^{n}
$$

A soma percorre todas as $2^{n}$ sequências $\left(\epsilon_{1}, \ldots, \epsilon_{n}\right)$, onde $\epsilon_{k}= \pm 1$ para cada $1 \leq k \leq n$. Demonstração. De acordo com a Fórmula de Leibniz 2.2.5, fixando-se $x_{0}, \ldots, x_{n} \in X$ e uma sequência $\left(\epsilon_{1}, \ldots, \epsilon_{n}\right)$, onde $\epsilon_{k}= \pm 1$ para cada $1 \leq k \leq n$, temos

$$
A\left(x_{0}+\epsilon_{1} x_{1}+\ldots+\epsilon_{n} x_{n}\right)^{n}=\sum_{|\alpha|=n} \frac{n !}{\alpha !} \epsilon_{1}^{\alpha_{1}} \ldots \epsilon_{n}^{\alpha_{n}} A x_{0}^{\alpha_{0}} \ldots x_{n}^{\alpha_{n}},
$$


equivalentemente

$$
\epsilon_{1} \ldots \epsilon_{n} A\left(x_{0}+\epsilon_{1} x_{1}+\ldots+\epsilon_{n} x_{n}\right)^{n}=\sum_{|\alpha|=n} \epsilon_{1}^{\alpha_{1}+1} \ldots \epsilon_{n}^{\alpha_{n}+1} \frac{n !}{\alpha !} A x_{0}^{\alpha_{0}} \ldots x_{n}^{\alpha_{n}}
$$

Considerando-se a soma percorrendo todas as $2^{n}$ sequências $\left(\epsilon_{1}, \ldots, \epsilon_{n}\right)$, onde $\epsilon_{k}= \pm 1$ para cada $1 \leq k \leq n$, teremos

$$
\begin{aligned}
\sum_{\epsilon_{k}= \pm 1} \epsilon_{1} \ldots \epsilon_{n} A\left(x_{0}+\epsilon_{1} x_{1}+\ldots+\epsilon_{n} x_{n}\right)^{n} & =\sum_{\epsilon_{k}= \pm 1} \sum_{|\alpha|=n} \epsilon_{1}^{\alpha_{1}+1} \ldots \epsilon_{n}^{\alpha_{n}+1} \frac{n !}{\alpha !} A x_{0}^{\alpha_{0}} \ldots x_{n}^{\alpha_{n}} \\
& =\sum_{|\alpha|=n} \frac{n !}{\alpha !} A x_{0}^{\alpha_{0}} \ldots x_{n}^{\alpha_{n}}\left(\sum_{\epsilon_{k}= \pm 1} \epsilon_{1}^{\alpha_{1}+1} \ldots \epsilon_{n}^{\alpha_{n}+1}\right) .
\end{aligned}
$$

Seja $\alpha=\left(\alpha_{0}, \ldots, \alpha_{n}\right) \in \mathbb{N}^{n+1}$ com $|\alpha|=n$. Se para algum $1 \leq k \leq n$ tivermos $\alpha_{k}=0$, e podemos supor sem perda de generalidade que $k=1$, então

$$
\begin{aligned}
\sum_{\epsilon_{k}= \pm 1} \epsilon_{1}^{\alpha_{1}+1} \ldots \epsilon_{n}^{\alpha_{n}+1} & =\sum_{\epsilon_{k}= \pm 1} \epsilon_{1} \epsilon_{2}^{\alpha_{2}+1} \ldots \epsilon_{n}^{\alpha_{n}+1} \\
& =\sum_{\epsilon_{k}= \pm 1} \epsilon_{2} \ldots \epsilon_{n}^{\alpha_{n}+1}-\sum_{\epsilon_{k}= \pm 1} \epsilon_{2} \ldots \epsilon_{n}^{\alpha_{n}+1}=0
\end{aligned}
$$

Lembrando-se que $|\alpha|=n$, podemos concluir que $\alpha_{k} \neq 0$ para todo $1 \leq k \leq n$ se e somente se $\alpha_{0}=0$ e $\alpha_{1}=\ldots=\alpha_{n}=1$. Então,

$$
\begin{aligned}
\sum_{\epsilon_{k}= \pm 1} \epsilon_{1} \ldots \epsilon_{n} A\left(x_{0}+\epsilon_{1} x_{1}+\ldots+\epsilon_{n} x_{n}\right)^{n} & =\left(\sum_{\epsilon_{k}= \pm 1} \epsilon_{1}^{2} \ldots \epsilon_{n}^{2}\right) n ! A\left(x_{1}, \ldots, x_{n}\right) \\
& =n ! 2^{n} A\left(x_{1}, \ldots, x_{n}\right),
\end{aligned}
$$

ou seja,

$$
A\left(x_{1}, \ldots, x_{n}\right)=\frac{1}{n ! 2^{n}} \sum_{\epsilon_{k}= \pm 1} \epsilon_{1} \ldots \epsilon_{n} A\left(x_{0}+\epsilon_{1} x_{1}+\ldots+\epsilon_{n} x_{n}\right)^{n} .
$$

\subsection{Polinômios}

Nesta seção estudaremos conceitos básicos sobre polinômios. Para um estudo mais detalhado sobre este tópico, recomendamos [17]. 
Definição 2.3.1. Dizemos que uma aplicação $P: X \rightarrow Y$ é um polinômio homogêneo de grau $n$, ou um polinômio $n$-homogêneo, se existir uma aplicação $A \in \mathcal{L}_{a}\left({ }^{n} X ; Y\right)$ satisfazendo

$$
P(x)=A x^{n}
$$

Denotaremos por $A$ a aplicação $n$-linear associada ao polinômio $P$.

Verifica-se facilmente que o conjunto dos polinômios $n$-homogêneos de $X$ em $Y$, munido das operações usuais de adição e multiplicação por escalar, é um espaço vetorial. Denotaremos esse espaço por $\mathcal{P}_{a}\left({ }^{n} X ; Y\right)$. Em particular, se $Y=\mathbb{K}$ denotaremos $\mathcal{P}_{a}\left({ }^{n} X\right):=$ $\mathcal{P}_{a}\left({ }^{n} X ; \mathbb{K}\right)$. Por convenção, $\mathcal{P}_{a}\left({ }^{0} X ; Y\right):=Y$.

Proposição 2.3.2. Sejam $n \in \mathbb{N}, P \in \mathcal{P}_{a}\left({ }^{n} X ; Y\right)$ e $A \in \mathcal{L}_{a}^{s}\left({ }^{n} X ; Y\right)$ a aplicação n-linear associada a P. São equivalentes:

(i) A é contínua;

(ii) Pé contínua;

(iii) P é contínua na origem;

(iv) Existe $M>0$ tal que $\|P(x)\| \leq M\|x\|^{n}$ para todo $x \in X$.

Demonstração. $(i) \Rightarrow($ ii $)$ Seja $I: X \rightarrow X^{n}$ a inclusão definida por

$$
I(x)=(\overbrace{x, \ldots, x}^{n}), x \in X
$$

Claramente, $I$ é contínua. Se $A$ for contínua então a composição $P=A \circ I$ é contínua.

$($ ii $) \Rightarrow($ iii) Evidente.

$(i i i) \Rightarrow($ iv $)$ Se $P$ for contínua na origem, então existe $\delta>0$ tal que $\|P(x)\| \leq 1$ sempre que $\|x\| \leq \delta$.

Se $x \neq 0$ então $\left\|\delta \frac{x}{\|x\|}\right\| \leq \delta$ e portanto

$$
\left\|P\left(\delta \frac{x}{\|x\|}\right)\right\| \leq 1
$$

Consequentemente

$$
\|P(x)\| \leq \frac{1}{\delta^{n}}\|x\|^{n}
$$

Claramente, a relação (2.4) é verdadeira quando $x=0$. 
$(i v) \Rightarrow(i)$ Seja $x=\left(x_{1}, \ldots, x_{n}\right)$ com $\|x\| \leq 1$ arbitrário. Utilizando-se a Fórmula de Polarização 2.2.7,

$$
\begin{aligned}
\left\|A\left(x_{1}, \ldots, x_{n}\right)\right\| & =\left\|\frac{1}{n ! 2^{n}} \sum_{\epsilon_{k}= \pm 1} \epsilon_{1} \ldots \epsilon_{n} A\left(\epsilon_{1} x_{1}+\ldots+\epsilon_{n} x_{n}\right)^{n}\right\| \\
& \leq \frac{1}{n ! 2^{n}} \sum_{\epsilon_{k}= \pm 1}\left\|P\left(\epsilon_{1} x_{1}+\ldots+\epsilon_{n} x_{n}\right)\right\| \\
& \leq \frac{M}{n ! 2^{n}} \sum_{\epsilon_{k}= \pm 1}\left\|\epsilon_{1} x_{1}+\ldots+\epsilon_{n} x_{n}\right\|^{n} \\
& \leq \frac{M}{n !}\left(\left\|x_{1}\right\|+\ldots+\left\|x_{n}\right\|\right)^{n} \\
& \leq \frac{M n^{n}}{n !}
\end{aligned}
$$

Portanto $\|A\|<\infty$, e de acordo com a proposição 2.1.2, $A$ é contínua.

Denotaremos por $\mathcal{P}\left({ }^{n} X ; Y\right)$ o subespaço de todos os polinômios $n$-homogêneos contínuos de $X$ em $Y$. Em particular, denotaremos $\mathcal{P}\left({ }^{n} X\right):=\mathcal{P}\left({ }^{n} X ; \mathbb{K}\right)$.

Proposição 2.3.3. A aplicação $\|\cdot\|: \mathcal{P}\left({ }^{n} X ; Y\right) \rightarrow \mathbb{R}$ definida por

$$
\|P\|:=\sup \{\|P(x)\|: x \in X,\|x\| \leq 1\}
$$

define uma norma sobre $\mathcal{P}\left({ }^{n} X ; Y\right)$.

Demonstração. Segue de forma análoga à proposição 2.1.5.

Proposição 2.3.4. Para cada $A \in \mathcal{L}\left({ }^{n} X ; Y\right)$ seja $\check{A} \in \mathcal{P}\left({ }^{n} X ; Y\right)$ definido por

$$
\check{A}(x):=A x^{n}, x \in X .
$$

Então:

(i) A aplicação $A \mapsto \check{A}$ é um isomorfismo entre $\mathcal{L}^{s}\left({ }^{n} X ; Y\right)$ e $\mathcal{P}\left({ }^{n} X ; Y\right)$;

(ii) Para cada $A \in \mathcal{L}^{s}\left({ }^{n} X ; Y\right)$ temos

$$
\|\check{A}\| \leq\|A\| \leq \frac{n^{n}}{n !}\|\check{A}\| .
$$


Demonstração. (i) Claramente aplicação $A \mapsto A$ é linear. Para demonstrarmos a sobrejetividade, fixemos $P \in \mathcal{P}\left({ }^{n} X ; Y\right)$ arbitrário. De acordo com a definição de polinômio $n$-homogêneo, e em virtude da proposição 2.3 .2 , existe $A \in \mathcal{L}\left({ }^{n} X ; Y\right)$ satisfazendo $P(x)=A x^{n}, x \in X$.

Seja $A^{s}$ a aplicação simétrica associada a $A$, ou seja, a aplicação

$$
A^{s}\left(x_{1}, \ldots, x_{n}\right):=\frac{1}{n !} \sum_{\sigma \in \mathcal{S}_{n}} A\left(x_{\sigma(1)}, \ldots, x_{\sigma(n)}\right) .
$$

Então, para todo $x \in X$

$$
A^{s} x^{n}=\frac{1}{n !} \sum_{\sigma \in \mathcal{S}_{n}} A(x, \ldots, x)=A x^{n}=P(x),
$$

e portanto $\check{A}^{s} \equiv P$, demonstrando que a aplicação é sobrejetora.

Para demonstrarmos a injetividade, seja $A \in \mathcal{L}^{s}\left({ }^{n} X ; Y\right)$ tal que $\check{A} \equiv 0$. De acordo com a Fórmula de Polarização 2.2.7, para todo $\left(x_{1}, \ldots, x_{n}\right) \in X^{n}$ temos

$$
A\left(x_{1}, \ldots, x_{n}\right)=\frac{1}{n ! 2^{n}} \sum_{\epsilon_{k} \pm 1} \epsilon_{1} \ldots \epsilon_{n} \check{A}\left(\epsilon_{1} x_{1}+\ldots+\epsilon_{n} x_{n}\right)^{n}=0,
$$

e portanto $A \equiv 0$, demonstrando que a aplicação é injetora.

(ii) Seja $x=\left(x_{1}, \ldots, x_{n}\right)$ tal que $\|x\| \leq 1$ arbitrário. De acordo com a Fórmula de Polarização 2.2.7,

$$
\begin{aligned}
\left\|A\left(x_{1}, \ldots, x_{n}\right)\right\| & =\left\|\frac{1}{n ! 2^{n}} \sum_{\epsilon_{k}= \pm 1} \epsilon_{1} \ldots \epsilon_{n} A\left(\epsilon_{1} x_{1}+\ldots+\epsilon_{n} x_{n}\right)^{n}\right\| \\
& \leq \frac{1}{n ! 2^{n}} \sum_{\epsilon_{k}= \pm 1}\left\|\check{A}\left(\epsilon_{1} x_{1}+\ldots+\epsilon_{n} x_{n}\right)\right\| \\
& \leq \frac{n^{n}\|\check{A}\|}{n ! 2^{n}} \sum_{\epsilon_{k}= \pm 1}\left(\frac{1}{n}\left\|\epsilon_{1} x_{1}+\ldots+\epsilon_{n} x_{n}\right\|\right)^{n} \\
& \leq \frac{n^{n}\|\check{A}\|}{n !}\left(\left\|\frac{x_{1}}{n}\right\|+\ldots+\left\|\frac{x_{n}}{n}\right\|\right)^{n} \leq \frac{n^{n}}{n !}\|\check{A}\| .
\end{aligned}
$$

Podemos concluir $\|A\| \leq \frac{n^{n}}{n !}\|\check{A}\|$. A outra desigualdade é evidente.

Mais geralmente, a aplicação $A \mapsto \check{A}$, associando cada $A \in \mathcal{L}_{a}\left({ }^{n} X ; Y\right)$ o polinômio 
$\check{A} \in \mathcal{P}_{a}\left({ }^{n} X ; Y\right)$, definido por

$$
\check{A}(x):=A x^{n}, x \in X,
$$

fornece um isomorfismo entre os espaços vetoriais $\mathcal{L}_{a}^{s}\left({ }^{n} X ; Y\right)$ e $\mathcal{P}_{a}\left({ }^{n} X ; Y\right)$.

Em diversos momentos neste trabalho, utilizaremos também a aplicação $P \rightarrow \widehat{P}$ que associa cada polinômio $P \in \mathcal{P}_{a}\left({ }^{n} X ; Y\right)$ a sua única aplicação $n$-linear simétrica associada, $\widehat{P} \in \mathcal{L}_{a}^{s}\left({ }^{n} X ; Y\right)$. De acordo com o que estudamos até agora, $P$ é contínua se e somente se $\widehat{P}$ for contínua.

Corolário 2.3.5. Se $Y$ é um espaço de Banach então, $\mathcal{P}\left({ }^{n} X ; Y\right)$ é um espaço de Banach.

Demonstração. De acordo com a proposição 2.3.4, os espaços $\mathcal{L}^{s}\left({ }^{n} X ; Y\right)$ e $\mathcal{P}\left({ }^{n} X ; Y\right)$ são isomomorfos e de acordo com o corolário 2.2.3, o espaço $\mathcal{L}^{s}\left({ }^{n} X ; Y\right)$ é completo. Decorre que $\mathcal{P}\left({ }^{n} X ; Y\right)$ é completo.

Definição 2.3.6. Dizemos que uma aplicação $P: X \rightarrow Y$ é um polinômio de $X$ em $Y$ se existir $n \in \mathbb{N}_{0}$ e $P_{k} \in \mathcal{P}_{a}\left({ }^{k} X ; Y\right), 0 \leq k \leq n$, tais que

$$
P=P_{0}+P_{1}+\ldots+P_{n}
$$

Claramente o conjunto dos polinômios de $X$ em $Y$, munido das operações usuais de adição e multiplicação por escalar, é um espaço vetorial. Denotaremos esse espaço por $\mathcal{P}_{a}(X ; Y)$. Em particular, denotaremos $\mathcal{P}_{a}(X):=\mathcal{P}_{a}(X ; \mathbb{K})$. Denotaremos por $\mathcal{P}(X ; Y)$ o subespaço de $\mathcal{P}_{a}(X ; Y)$ de todas os polinômios contínuos de $X$ em $Y$. Em particular, $\mathcal{P}(X):=\mathcal{P}(X ; \mathbb{K})$.

\section{Proposição 2.3.7.}

(i) $\mathcal{P}_{a}(X ; Y)$ é a soma direta dos subespaços $\mathcal{P}_{a}\left({ }^{n} X ; Y\right), n \in \mathbb{N}$.

(ii) $\mathcal{P}(X ; Y)$ é a soma direta dos subespaços $\mathcal{P}\left({ }^{n} X ; Y\right), n \in \mathbb{N}$.

Demonstração. Fixemos $P=P_{0}+P_{1}+\ldots+P_{n} \in \mathcal{P}(X ; Y)$ onde $P_{k} \in \mathcal{P}\left({ }^{k} X ; Y\right), 0 \leq k \leq n$.

Para verificarmos $(i)$, basta demonstrar que se $P=0$ então $P_{0}=P_{1}=\ldots=P_{n}=0$. De fato, se $P=0$ então para cada $x \in X$ e cada $\lambda \in \mathbb{K}$

$$
\sum_{k=0}^{n} \lambda^{k} P_{k}(x)=\sum_{k=0}^{n} P_{k}(\lambda x)=0 .
$$


Fixando-se $\lambda=1,2, \ldots, n+1$, podemos escrever para todo $x \in X$

$$
\left(\begin{array}{ccccc}
1 & 1 & 1 & \ldots & 1 \\
1 & 2 & 2^{2} & \ldots & 2^{n} \\
1 & 3 & 3^{2} & \ldots & 3^{n} \\
\vdots & \vdots & \vdots & \ddots & \vdots \\
1 & n+1 & (n+1)^{2} & \ldots & (n+1)^{n}
\end{array}\right)\left(\begin{array}{c}
P_{0}(x) \\
P_{1}(x) \\
P_{2}(x) \\
\vdots \\
P_{n}(x)
\end{array}\right)=\left(\begin{array}{c}
0 \\
0 \\
0 \\
\vdots \\
0
\end{array}\right) .
$$

A matriz quadrada no primeiro membro de 2.5 é claramente invertível (matriz de Vandermonde). Decorre

$$
P_{0}(x)=P_{1}(x)=\ldots P_{n}(x)=0,
$$

e isso verifica $(i)$.

Para verificarmos (ii) precisamos apenas demonstrar que $P_{k}$ é contínua para cada $k$, e faremos isso por indução em $n$.

Claramente a tese se verifica no caso em que $n=0$. Se $n \geq 1$ suponha a tese válida para todo $k \leq n-1$. Para cada cada $x \in X$ e para cada $\lambda \in \mathbb{K}$ temos

$$
P(\lambda x)-\lambda^{n} P(x)=\sum_{k=0}^{n-1}\left(\lambda^{k}-\lambda^{n}\right) P_{k}(x) .
$$

Fixando-se $\lambda \in \mathbb{K}$ tal que $\left(\lambda^{k}-\lambda^{n}\right) \neq 0$ para todo $0 \leq k \leq n-1$, a aplicação $Q: X \rightarrow \mathbb{K}$ definida por

$$
Q(x):=P(\lambda x)-\lambda^{n} P(x), x \in X,
$$

é um polinômio contínuo de grau estritamente menor que $n$. De acordo com a hipótese de indução, $P_{k}$ é contínuo para todo $0 \leq k \leq n-1$, consequentemente $P_{n}=P-P_{0}-\ldots-P_{n-1}$ também é contínua. Isso verifica (ii).

Como no caso homogêneo, a aplicação $\|\cdot\|: \mathcal{P}(X ; Y) \rightarrow \mathbb{R}$ definida por

$$
\|P\|:=\sup \{\|P(x)\|: x \in X,\|x\| \leq 1\},
$$

define uma norma sobre $\mathcal{P}(X ; Y)$.

Demonstra-se que, sob a topologia induzida por essa norma, $\mathcal{P}(X ; Y)$ é espaço de Banach. Definiremos agora a derivada de um polinômio homogêneo.

Definição 2.3.8. Sejam $P \in \mathcal{P}\left({ }^{n} X ; Y\right)$ e um natural $1 \leq k \leq n$. A $k$-ésima derivada de $P$ 
é a aplicação $\partial^{k} P \in \mathcal{P}\left({ }^{n-k} X ; \mathcal{P}\left({ }^{k} X ; Y\right)\right)$ definida por

$$
\partial^{k} P(x)(y):=\frac{n !}{(n-k) !} \widehat{P} x^{n-k} y^{k}, x, y \in X .
$$

A proposição a seguir terá um papel importante no capítulo 3.

Proposição 2.3.9. Para todo $P \in \mathcal{P}\left({ }^{n} X\right)$, ker $\partial^{n-1} P=\partial P(X)_{\perp}$.

Demonstração. Notando-se que para quaisquer $x, y \in X$,

$$
\partial^{n-1} P(x)(y)=n ! \widehat{P} x y^{n-1}=(n-1) ! n \widehat{P} y^{n-1} x=(n-1) ! \partial P(y)(x),
$$

temos $x \in \operatorname{ker} \partial^{n-1} P$ se e somente se $x \in \partial P(X)_{\perp}$.

\subsection{Polinômios Fracamente Contínuos sobre Limitados}

Nesta seção estudaremos alguns tipos especiais de polinômios. Exibiremos algumas definições e resultados importantes para o desenvolvimento do capítulo 3. Esta seção é baseada principalmente em [9].

Definição 2.4.1. Dizemos que um polinômio $P \in \mathcal{P}\left({ }^{n} X ; Y\right)$ é fracamente contínuo, ou wcontínuo, sobre os subconjuntos limitados de $X$, se para cada subconjunto limitado $A \subset X$, a restrição

$$
P:\left(A ; \sigma\left(X ; X^{*}\right)\right) \rightarrow(Y ;\|.\|) .
$$

for contínua.

Verifica-se que o conjunto dos polinômios fracamente contínuos sobre os limitados é um subespaço de $\mathcal{P}\left({ }^{n} X, Y\right)$. Denotaremos esse subespaço por $\mathcal{P}_{w}\left({ }^{n} X ; Y\right)$. Em particular denotaremos $\mathcal{P}_{w}\left({ }^{n} X\right):=\mathcal{P}_{w}\left({ }^{n} X, \mathbb{K}\right)$.

Mais geralmente, uma função $f: X \rightarrow Y$ é fracamente contínua sobre os subconjuntos limitados de $X$ se para cada subconjunto limitado $A \subset X$, a restrição

$$
f:\left(A ; \sigma\left(X ; X^{*}\right)\right) \rightarrow(Y ;\|.\|) .
$$

for contínua. 
Dizemos também que uma função $f: X \rightarrow Y$ é fracamente uniformemente contínua sobre os subconjuntos limitados de $X$ se para cada subconjunto limitado $A \subset X$, a restrição

$$
f:\left(A ; \sigma\left(X ; X^{*}\right)\right) \rightarrow(Y ;\|\cdot\|) .
$$

for uniformemente contínua.

Definição 2.4.2. Dizemos que um polinômio $P \in \mathcal{P}\left({ }^{n} X ; Y\right)$ é fracamente sequencialmente contínuo, ou wsc-contínuo, se a sequência $\left(P\left(x_{k}\right)\right)_{k \in \mathbb{N}}$ converge em norma a $P(x)$ sempre que a sequência $\left(x_{k}\right)_{k \in \mathbb{N}}$ converge fracamente para $x \in X$.

Verifica-se que o conjunto dos polinômios fracamente sequencialmente contínuos é um subespaço de $\mathcal{P}\left({ }^{n} X, Y\right)$. Este espaço será denotado por $\mathcal{P}_{w s c}\left({ }^{n} X ; Y\right)$. Em particular denotaremos $\mathcal{P}_{\text {wsc }}\left({ }^{n} X\right):=\mathcal{P}_{\text {wsc }}\left({ }^{n} X, \mathbb{K}\right)$.

Definição 2.4.3. Dizemos que um polinômio $P \in \mathcal{P}\left({ }^{n} X ; Y\right)$ é nuclear se existirem sequências limitadas $\left(\varphi_{k}\right)_{k \in \mathbb{N}} \subset X^{*},\left(y_{k}\right)_{k \in \mathbb{N}} \subset Y$ e $\left(\lambda_{k}\right)_{k \in \mathbb{N}} \in l_{1}$ tais que

$$
P(x)=\sum_{k=1}^{\infty} \lambda_{k} \varphi_{k}^{n}(x) y_{k}, x \in X
$$

O conjunto dos polinômios nucleares é um subespaço de $\mathcal{P}\left({ }^{n} X, Y\right)$. Denotaremos este subespaço por $\mathcal{P}_{N}\left({ }^{n} X, Y\right)$. Em particular $\mathcal{P}_{N}\left({ }^{n} X\right):=\mathcal{P}_{N}\left({ }^{n} X, \mathbb{K}\right)$.

Proposição 2.4.4. $P \in \mathcal{P}_{w}\left({ }^{n} X ; Y\right)$ se e somente se $P$ é w-contínuo sobre a bola unitária $B_{X}$.

Demonstração. Seja $P \in \mathcal{P}\left({ }^{n} X ; Y\right)$ arbitrário. Claramente, se $P$ for $w$-contínuo sobre os subconjuntos limitados de $X$, será $w$-contínuo sobre $B_{X}$.

Por outro lado, dado um subconjunto limitado não vazio $K \subset X$, existe $\delta>0$ tal que $\delta K \subset B_{X}$.

Seja $\left(x_{\gamma}\right)_{\gamma \in \Gamma}$ for uma rede arbitrária em $K$ convergindo fracamente a algum $x \in K$, segue-se que $\left(\delta x_{\gamma}\right)_{\gamma \in \Gamma}$ será uma rede em $B_{X}$ convergindo fracamente a $\delta x \in B_{X}$.

Se $P$ for $w$-contínuo sobre $B_{X}$, de acordo com o teorema 1.1.3, a rede $\left(P\left(\delta x_{\gamma}\right)\right)_{\gamma \in \Gamma}$ converge em norma a $P(\delta x)$. Por $P\left(\delta x_{\gamma}\right)=\delta^{n} P\left(x_{\gamma}\right), \gamma \in \Gamma$, a rede $\left(P\left(x_{\gamma}\right)\right)_{\gamma \in \Gamma}$ converge em norma a $P(x)$. Por $\left(x_{\gamma}\right)_{\gamma \in \Gamma}$ ser uma rede arbitrária em $K$, o teorema 1.1.3 implica que o polinômio $P$ é fracamente contínuo sobre $K$, e como $K$ é um subconjunto limitado arbitrário de $X$, concluímos que $P \in \mathcal{P}_{w}\left({ }^{n} X ; Y\right)$. 
Proposição 2.4.5. $\mathcal{P}_{N}\left({ }^{n} X ; Y\right) \subseteq \mathcal{P}_{w}\left({ }^{n} X ; Y\right) \subseteq \mathcal{P}_{w s c}\left({ }^{n} X ; Y\right)$.

Demonstração. Verificaremos primeiramente que $\mathcal{P}_{N}\left({ }^{n} X ; Y\right) \subseteq \mathcal{P}_{w}\left({ }^{n} X ; Y\right)$. Fixemos um polinômio nuclear $P(x)=\sum_{k=1}^{\infty} \lambda_{k} \varphi_{k}^{n}(x) y_{k}$, onde $\left(\varphi_{k}\right)_{k \in \mathbb{N}}$ e $\left(y_{k}\right)_{k \in \mathbb{N}}$ são sequências limitadas em $X^{*}$ e $Y$ respectivamente, e $\left(\lambda_{k}\right)_{k \in \mathbb{N}} \in l_{1}$. Seja $M>0$, uma constante satisfazendo

$$
\max \left\{\sup _{k \in \mathbb{N}}\left\|\varphi_{k}\right\|, \sup _{k \in \mathbb{N}}\left\|y_{k}\right\|\right\} \leq M .
$$

Seja $\left(x_{\gamma}\right)_{\gamma \in \Gamma}$ uma rede arbitrária em $B_{X}$ convergindo fracamente a algum $x \in B_{X}$ e fixemos $\epsilon>0$ arbitrário. Por $\sum_{r=1}^{\infty}\left|\lambda_{k}\right|:=\alpha<\infty$, existe $k_{0} \in \mathbb{N}$ tal que

$$
\sum_{r=k}^{\infty}\left|\lambda_{r}\right|<\frac{\epsilon}{4} \frac{1}{M^{n+1}}, \text { sempre que } k \geq k_{0} .
$$

Por $\left(x_{\gamma}\right)_{\gamma \in \Gamma}$ convergir fracamente a $x$, para cada $1 \leq k \leq k_{0}$ existe $\gamma_{k} \in \Gamma$ tal que

$$
\left|\varphi_{k}\left(x_{\gamma}\right)-\varphi_{k}(x)\right| \leq \frac{\epsilon}{n\left(\left\|\varphi_{k}\right\|+1\right)^{n-1}} \frac{1}{2(\alpha+1) M k_{0}},
$$

sempre que $\gamma \geq \gamma_{k}$. Consequentemente

$$
\begin{aligned}
\left|\varphi_{k}^{n}\left(x_{\gamma}\right)-\varphi_{k}^{n}(x)\right| & =\left|\sum_{r=1}^{n}\left(\varphi_{k}^{n-r+1}\left(x_{\gamma}\right) \varphi_{k}^{r-1}(x)-\varphi_{k}^{n-r}\left(x_{\gamma}\right) \varphi_{k}^{r}(x)\right)\right| \\
& =\left|\sum_{r=1}^{n} \varphi_{k}^{n-r}\left(x_{\gamma}\right) \varphi_{k}^{r-1}(x)\left(\varphi_{k}\left(x_{\gamma}\right)-\varphi_{k}(x)\right)\right| \\
& \leq \sum_{r=1}^{n}\left|\varphi_{k}^{n-r}\left(x_{\gamma}\right) \varphi_{k}^{r-1}(x)\left(\varphi_{k}\left(x_{\gamma}\right)-\varphi_{k}(x)\right)\right| \\
& \leq n\left\|\varphi_{k}\right\|^{n-1}\left|\varphi_{k}\left(x_{\gamma}\right)-\varphi_{k}(x)\right| \\
& <\frac{\epsilon}{2(\alpha+1) M k_{0}} .
\end{aligned}
$$

Por $\Gamma$ ser um conjunto dirigido, existe $\gamma_{0} \in \Gamma$ tal que $\gamma_{0} \geq \gamma_{k}$ para todo $1 \leq k \leq k_{0}$. 
Então, sempre que $\gamma \geq \gamma_{0}$

$$
\begin{aligned}
\left\|P\left(x_{\gamma}\right)-P(x)\right\| & =\left\|\sum_{r=1}^{\infty} \lambda_{r} \varphi_{k}^{n}\left(x_{\gamma}\right) y_{r}-\sum_{r=1}^{\infty} \lambda_{r} \varphi_{k}^{n}(x) y_{r}\right\| \\
& \leq \sum_{r=1}^{k_{0}}\left|\lambda_{r}\right|\left|\varphi_{k}^{n}\left(x_{\gamma}\right)-\varphi_{k}^{n}(x)\right|\left\|y_{r}\right\|+\sum_{r=k_{0}+1}^{\infty}\left|\lambda_{r}\right|\left|\varphi_{k}^{n}\left(x_{\gamma}\right)-\varphi_{k}^{n}(x)\right|\left\|y_{r}\right\| \\
& \leq \alpha M \sum_{r=1}^{k_{0}}\left|\varphi_{k}^{n}\left(x_{\gamma}\right)-\varphi_{k}^{n}(x)\right|+2 M^{n+1} \sum_{r=k_{0}+1}^{\infty}\left|\lambda_{r}\right| \\
& <\alpha M k_{0}\left(\frac{\epsilon}{2(\alpha+1) M k_{0}}\right)+2 M^{n+1}\left(\frac{\epsilon}{4} \frac{1}{M^{n+1}}\right) \\
& \leq \epsilon .
\end{aligned}
$$

Consequentemente, a rede $\left(P\left(x_{\gamma}\right)\right)_{\gamma \in \Gamma}$ converge em norma a $P(x)$. Em virtude do teorema 1.1.3, concluímos que $P$ é $w$-contínua sobre $B_{X}$, e em virtude da proposição 2.4.4, decorre $P \in \mathcal{P}_{w}\left({ }^{m} X ; Y\right)$.

Fixemos agora $P \in \mathcal{P}_{w}\left({ }^{n} X ; Y\right)$. Seja $\left(x_{k}\right)_{k \in \mathbb{N}}$ uma sequência arbitrária em $X$ convergindo fracamente a algum $x \in X$. Claramente a sequência é fracamente limitada, portanto, de acordo com a proposição 1.2.9, é limitada em norma. Por $P$ ser fracamente contínuo sobre os limitados de $X$, a sequência $\left(P\left(x_{k}\right)\right)_{k \in \mathbb{N}}$ converge em norma a $P(x)$. Concluímos $P \in$ $\mathcal{P}_{w s c}\left({ }^{n} X ; Y\right)$.

O teorema a seguir estabelece uma condição necessária e suficiente sobre um espaço $X$, para que $\mathcal{P}_{w s c}\left({ }^{n} X\right) \subseteq \mathcal{P}_{w}\left({ }^{n} X\right)$. A demonstração deste resultado pode ser encontrada em [9] (pág. 116). Diremos que um espaço de Banach contém cópia de $l_{1}$ se contém subespaço isomorfo a $l_{1}$.

Proposição 2.4.6. Se $X$ é um espaço de Banach então $\mathcal{P}_{w s c}\left({ }^{n} X\right) \subseteq \mathcal{P}_{w}\left({ }^{n} X\right)$ se e somente se $X$ não contém cópia de $l_{1}$.

Teorema 2.4.7. Seja $A \in \mathcal{L}\left({ }^{n} X ; Y\right)$, onde $X$ e $Y$ são espaços de Banach. As seguintes afirmações são equivalentes:

(i) A é fracamente contínua sobre os limitados.

(ii) Se $\left(x_{\alpha}^{k}\right)_{\alpha \in \Gamma}, 1 \leq k \leq n$ forem redes $w$-Cauchy limitadas tais que ao menos uma convirja fracamente a zero, então $\left(A\left(x_{\alpha}^{1}, \ldots, x_{\alpha}^{n}\right)\right)_{\alpha \in \Gamma}$ converge a zero.

(iii) Se $\left(x_{\alpha}^{k}\right)_{\alpha \in \Gamma}, 1 \leq k \leq n$ forem redes de $w$-Cauchy limitadas então $\left(A\left(x_{\alpha}^{1}, \ldots, x_{\alpha}^{n}\right)\right)_{\alpha \in \Gamma}$ é uma rede de Cauchy em $Y$. 
(iv) A é fracamente uniformemente contínua sobre os limitados.

A demonstração do teorema 2.4 .7 pode ser encontrada em [9](pág. 87). Este teorema é essencial para a verificação da proposição a seguir, que por sua vez terá um papel importante no capítulo 3.

Proposição 2.4.8. Se $P \in \mathcal{P}\left({ }^{n} X ; Y\right)$ onde $X$ e $Y$ são espaços de Banach, então as seguintes afirmações são equivalentes:

(i) $P \in \mathcal{P}_{w}\left({ }^{n} X ; Y\right)$;

(ii) P é fracamente uniformemente contínuo sobre os subconjuntos limitados de $X$;

(iii) Para cada $0 \leq k \leq n$, a aplicação $\partial^{k} P$ é fracamente uniformemente contínua sobre os subconjuntos limitados de $X$.

Demonstração. (i) $\Rightarrow$ (ii) Em virtude da Fórmula de Polarização 2.2.7, $P \in \mathcal{P}\left({ }^{n} X ; Y\right)$ é fracamente uniformemente contínuo sobre os limitados se e somente se $\widehat{P} \in \mathcal{L}_{s}\left({ }^{n} X ; Y\right)$ possuir a mesma propriedade. A tese, por sua vez, decorre imediatamente do teorema 2.4.7.

(ii) $\Rightarrow$ (iii) Suponha para algum $1 \leq k \leq n, \partial^{k} P$ não seja fracamente uniformemente contínua sobre limitados de $X$. Então $\partial^{k} P$ não será fracamente uniformemente contínua sobre $B_{X}$, de onde existem $\epsilon>0$ e redes $w$-Cauchy, $\left(x_{\gamma}\right)_{\gamma \in \Gamma}$ e $\left(y_{\gamma}\right)_{\gamma \in \Gamma}$ em $B_{X}$ satisfazendo

$$
x_{\gamma}-y_{\gamma} \rightarrow 0 \text { e }\left\|\partial^{k} P\left(x_{\gamma}\right)-\partial^{k} P\left(x_{\gamma}\right)\right\| \geq \epsilon \text { para todo } \gamma \in \Gamma \text {. }
$$

Para cada $\gamma \in \Gamma$ seja $z_{\gamma} \in B_{X}$ tal que

$$
\left\|\partial^{k} P\left(x_{\gamma}\right)\left(z_{\gamma}\right)-\partial^{k} P\left(y_{\gamma}\right)\left(z_{\gamma}\right)\right\| \geq \frac{\epsilon}{2}
$$

e fixemos a rede $\left(z_{\gamma}\right)_{\gamma \in \Gamma}$ em $B_{X}$.

Segundo a proposição 1.3.7, a rede $\left(z_{\gamma}\right)_{\gamma \in \Gamma}$ admite uma subrede $w$-Cauchy que, por simplicidade, denotaremos por $\left(z_{\gamma}\right)_{\gamma \in \Gamma^{\prime}}$. Para cada $\gamma \in \Gamma^{\prime}$, a Fórmula do Binômio 2.2.6 nos permite obter a seguinte identidade,

$$
\begin{aligned}
\partial^{k} P\left(x_{\gamma}\right)\left(z_{\gamma}\right)-\partial^{k} P\left(y_{\gamma}\right)\left(z_{\gamma}\right) & =k !\left(\begin{array}{l}
n \\
k
\end{array}\right)\left(\widehat{P} x_{\gamma}^{n-k} z_{\gamma}^{k}-\widehat{P} y_{\gamma}^{n-k} z_{\gamma}^{k}\right) \\
& =k !\left(\begin{array}{l}
n \\
k
\end{array}\right) \sum_{r=0}^{n-k-1}\left(\begin{array}{c}
n-k \\
r
\end{array}\right) \widehat{P}\left(\left(x_{\gamma}-y_{\gamma}\right)^{n-k-r}, y_{\gamma}^{r}, z_{\gamma}^{k}\right) .
\end{aligned}
$$

Por $P$ ser fracamente uniformemente contínuo sobre os limitados, a Fórmula de Polarização implica que $\widehat{P} \in \mathcal{L}_{s}\left({ }^{n} X ; Y\right)$ possui a mesma propriedade. 
Em virtude de $\left(x_{\gamma}-y_{\gamma}\right)_{\gamma \in \Gamma^{\prime}},\left(y_{\gamma}\right)_{\gamma \in \Gamma^{\prime}}$ e $\left(z_{\gamma}\right)_{\gamma \in \Gamma^{\prime}}$ serem redes $w$-Cauchy limitadas com $\left(x_{\gamma}-y_{\gamma}\right)_{\gamma \in \Gamma^{\prime}}$ convergindo fracamente a 0 , a proposição 2.4 .7 e a identidade acima implicam

$$
\partial^{k} P\left(x_{\gamma}\right)\left(z_{\gamma}\right)-\partial^{k} P\left(y_{\gamma}\right)\left(z_{\gamma}\right) \rightarrow 0,
$$

contradizendo 2.6 .

$($ iii $) \Rightarrow($ i $)$ Analogamente à primeira parte da demonstração, em virtude da Fórmula de Polarização 2.2.7, $P \in \mathcal{P}\left({ }^{n} X ; Y\right)$ é fracamente uniformemente contínua sobre os limitados se e somente se $\widehat{P} \in \mathcal{L}_{s}\left({ }^{n} X ; Y\right)$ possuir a mesma propriedade. A tese, por sua vez, decorre escolhendo-se $k=0$ e aplicando-se novamente a proposição 2.4.7.

O resultado a seguir será importante no estudo de zeros de polinômios, no capítulo 3.

Proposição 2.4.9. Se $P \in \mathcal{P}_{w}\left({ }^{n} X ; Y\right)$ onde onde $X$ e $Y$ são espaços de Banach, então para cada $0 \leq k \leq n$, o conjunto $\partial^{k} P(X)$ é separável em $\mathcal{P}\left({ }^{k} X ; Y\right)$.

Demonstração. Seja $\left(y_{\alpha}\right)_{\alpha \in \Gamma}$ uma rede em $\partial^{k} P\left(B_{X}\right)$ arbitrária e $\left(x_{\alpha}\right)_{\alpha \in \Gamma}$ uma rede em $B_{X}$ tal que $y_{\alpha}=\partial^{k} P\left(x_{\alpha}\right)$ para todo $\alpha \in \Gamma$.

De acordo com a proposição 1.3.7, $B_{X}$ é $w$-precompacto. Então $\left(x_{\alpha}\right)_{\alpha \in \Gamma}$ admite uma subrede $w$-Cauchy, que por simplicidade, denotaremos $\left(x_{\alpha}\right)_{\alpha \in \Gamma^{\prime}}$.

Em virtude das proposições 2.4 .8 e 1.3.5, a subrede $\left(y_{\alpha}\right)_{\alpha \in \Gamma^{\prime}}$ é de Cauchy. Concluímos que toda rede em $\partial^{k} P\left(B_{X}\right)$ admite subrede de Cauchy e consequentemente $\partial^{k} P\left(B_{X}\right)$ é precompacto em $\mathcal{P}\left({ }^{k} X ; Y\right)$. Então, pela proposição 1.3.6, $\partial^{k} P\left(B_{X}\right)$ é separável em $\mathcal{P}\left({ }^{k} X ; Y\right)$. Notando-se que

$$
\partial^{k} P(X)=\bigcup_{n \in \mathbb{N}} n \partial^{k} P\left(B_{X}\right)
$$

concluímos que $\partial^{k} P(X)$ é separável em $\mathcal{P}\left({ }^{k} X ; Y\right)$. 


\section{Capítulo 3}

\section{Zeros de Polinômios}

Em seu artigo [22], A. Plichko e A. Zagorodnyuk demonstraram que sobre um espaço de Banach complexo de dimensão infinita, todo polinômio $n$-homogêneo assumindo valores complexos se anula em um subespaço de dimensão infinita. Este artigo despertou grande interesse no estudo de zeros de polinômios $n$-homogêneos, em diversas direções. Nosso foco será o estudo de condições sob as quais um resultado similar seja válido para espaços de Banach reais de dimensão infinita. Diversos estudos já foram feitos nessa direção, mas este problema não está completamente resolvido.

Nesse trabalho estudamos técnicas desenvolvidas por J. Ferrer em [11] e algumas aplicações que resolvem parcialmente este problema para um particular tipo de polinômio em um particular tipo de espaço.

Neste capítulo, $X$ denotará sempre um espaço de Banach, embora alguns resultados, como por exemplo o teorema 3.1.1, dependam apenas da estrutura algébrica de espaço vetorial.

\subsection{Zeros de Polinômios em Espaços de Banach Complexos}

Seja $c_{0}$ o espaço das sequências em $\mathbb{K}$, convergindo a zero. Fixemos o polinômio $P$ definido por

$$
P\left(\left(x_{n}\right)_{n \in \mathbb{N}}\right):=\sum_{n=1}^{\infty} \frac{1}{2^{n}} x_{n}^{2} .
$$

Se $\mathbb{K}=\mathbb{R}$ verifica-se facilmente que $P\left(\left(x_{n}\right)_{n \in \mathbb{N}}\right)=0$ se e somente se $x_{n}=0$ para todo $n$. Por outro lado se $\mathbb{K}=\mathbb{C}$ e $\left\{e_{n}: n \in \mathbb{N}\right\}$ for a base canônica de $c_{0}$, claramente $\left\{e_{2 n}+\sqrt{2} \mathbf{i} e_{2 n+1}: n \in \mathbb{N}\right\}$ é um conjunto lineramente independente infinito e para cada $u=$ 
$a_{1}\left(e_{2 n_{1}}+\sqrt{2} \mathbf{i} e_{2 n_{1}+1}\right)+\ldots+a_{r}\left(e_{2 n_{r}}+\sqrt{2} \mathbf{i} e_{2 n_{r}+1}\right) \operatorname{com} a_{1}, \ldots, a_{r} \in \mathbb{C}$,

$$
P(u)=\sum_{k=1}^{r}\left(\frac{a_{k}^{2}}{2^{2 n_{k}}}+\frac{a_{k}^{2}(\sqrt{2} \mathbf{i})^{2}}{2^{2 n_{k}+1}}\right)=\sum_{k=1}^{r}\left(\frac{a_{k}^{2}}{2^{2 n_{k}}}-\frac{a_{k}^{2}}{2^{2 n_{k}}}\right)=0 .
$$

Concluímos que $\left[\left\{e_{2 n}+\sqrt{2} \mathbf{i} e_{2 n+1}: n \in \mathbb{N}\right\}\right]$ é um espaço de dimensão infinita contido em $P^{-1}(0)$. Mais geralmente vale o seguinte teorema, publicado em [22].

Teorema 3.1.1. (A. Plichko, A. Zagorodnyuk) Sejam X um espaço de Banach complexo e $P \in \mathcal{P}_{a}\left({ }^{n} X\right)$. Se $X$ tem dimensão infinita então $P^{-1}(0)$ contém um subespaço de dimensão infinita.

Demonstração. Por indução sobre $n$, construiremos um subespaço $Z \subset P^{-1}(0)$ com dimensão infinita.

Se $n=1$ então $P$ é um funcional linear e a tese é trivialmente verificada.

Supondo a tese verdadeira para todo $k \leq n$, fixemos $x_{1} \in X$ tal que $P\left(x_{1}\right) \neq 0$ (se não existir $x_{1}$ satisfazendo essa condição, $P \equiv 0$ e a tese está verificada).

Para cada $1 \leq k \leq n-1$ sejam $P_{x_{1}^{k}} \in \mathcal{P}\left({ }^{n-k} X\right)$ os polinômios homogêneos definidos por

$$
P_{x_{1}^{k}}(x):=\widehat{P}(\overbrace{x_{1}, \ldots, x_{1}}^{k}, x, \ldots, x), x \in X .
$$

Segundo a hipótese de indução existe um subespaço de dimensão infinita $Y_{1} \subset P_{x_{1}^{1}}^{-1}(0)$. Considerando-se $P_{x_{1}^{2}} \uparrow_{Y_{1}}$, novamente pela hipótese de indução, existe um subespaço de dimensão infinita $Y_{2} \subset P_{x_{1}^{2}}^{-1}(0) \cap Y_{1}$. Continuando esse processo obtemos um subespaço $Z_{1}:=Y_{n-1}$ de dimensão infinita satisfazendo

$$
Z_{1} \subset P_{x_{1}^{n-1}}^{-1}(0) \cap Y_{n-2} \subset P_{x_{1}^{n-1}}^{-1}(0) \cap P_{x_{1}^{n-2}}^{-1}(0) \cap Y_{n-3} \subset \ldots \subset P_{x_{1}^{n-1}}^{-1}(0) \cap \ldots \cap P_{x_{1}^{1}}^{-1}(0) .
$$

Fixemos $x_{2} \in Z_{1}$ tal que $P\left(x_{2}\right) \neq 0$ (se não existir $x_{2}$ satisfazendo essa condição, teremos $Z_{1} \subset P^{-1}(0)$ e consequentemente a tese).

Para cada $1 \leq k+l \leq n-1$ sejam $P_{x_{1}^{k} x_{2}^{l}} \in \mathcal{P}\left({ }^{n-k-l} Z_{1}\right)$ os polinômios homogêneos definidos por

$$
P_{x_{1}^{k} x_{2}^{l}}(x):=\widehat{P}(\overbrace{x_{1}, \ldots, x_{1}}^{k}, \overbrace{x_{2} \ldots, x_{2}}^{l}, x, \ldots, x) .
$$

Procedendo-se como acima obtemos um subespaço de dimensão infinita $Z_{2} \subset Z_{1}$ tal que

$$
Z_{2} \subset \bigcap_{1 \leq k+l \leq n-1} P_{x_{1}^{k} x_{2}^{l}}^{-1}(0)
$$


Fixemos agora $x_{3} \in Z_{2}$ tal que $P\left(x_{3}\right) \neq 0$ (se não existir $x_{3}$ satisfazendo essa condição, teremos $Z_{2} \subset P^{-1}(0)$ e consequentemente a tese).

Para cada $1 \leq k+l+m \leq n-1$ sejam $P_{x_{1}^{k} x_{2}^{l} x_{3}^{m}} \in \mathcal{P}\left({ }^{n-k-l-m} Z_{2}\right)$ os polinômios homogêneos definidos por

$$
P_{x_{1}^{k} x_{2}^{l} x_{3}^{m}}(x):=\widehat{P}(\overbrace{x_{1}, \ldots, x_{1}}^{k}, \overbrace{x_{2}, \ldots, x_{2}}^{l}, \overbrace{x_{3}, \ldots, x_{3}}^{m}, x, \ldots, x) .
$$

Procedendo-se como anteriormente obtemos um subespaço de dimensão infinita $Z_{3} \subset Z_{2}$ tal que

$$
Z_{3} \subset \bigcap_{1 \leq k+l+m \leq n-1} P_{x_{1}^{k} x_{2}^{l} x_{3}^{m}}^{-1}(0)
$$

Continuando dessa maneira, se para algum $r \in \mathbb{N}, Z_{r} \subset P^{-1}(0)$, o processo termina e o teorema está demonstrado. Por outro lado, se este processo nunca termina, denominando-se $Z_{0}:=X$, por construção, para cada $k \in \mathbb{N}, Z_{k-1} \subset Z_{k}$ e $x_{k} \in Z_{k-1} \backslash Z_{k}$ e portanto a sequência $\left(x_{k}\right)_{k \in \mathbb{N}}$ forma um conjunto linearmente independente tal que $P\left(x_{k}\right) \neq 0$ para todo $k$ e quaisquer que sejam $r_{1}, \ldots, r_{s} \in \mathbb{N}$,

$$
\widehat{P}(\overbrace{x_{r_{1}}, \ldots, x_{r_{1}}}^{\alpha_{1}}, \overbrace{x_{r_{2}}, \ldots, x_{r_{2}}}^{\alpha_{2}}, \ldots, \overbrace{x_{r_{s}}, \ldots, x_{r_{s}}}^{\alpha_{s}})=0
$$

se ao menos algum $1 \leq \alpha_{i} \leq n-1$.

De acordo com a Fórmula de Leibniz 2.2.5,

$P\left(\sum_{i=1}^{r} a_{i} x_{i}\right)=\widehat{P}\left(\sum_{i=1}^{r} a_{i} x_{i}\right)^{n}=\sum_{\alpha_{1}+\ldots+\alpha_{r}=n} \frac{n !}{\alpha_{1} ! \ldots \alpha_{r} !} a_{1}^{\alpha_{1}} \ldots a_{r}^{\alpha_{r}} \widehat{P} x_{1}^{\alpha_{1}} \ldots x_{r}^{\alpha_{r}}=\sum_{i=1}^{r} a_{i}^{n} P\left(x_{i}\right)$.

Fixando-se $\xi \in \mathbb{C}$ tal que $\xi^{n}+1=0$, seja $\left(y_{k}\right)_{k \in \mathbb{N}}$ a sequência definida por

$$
y_{k}:=\frac{x_{2 k-1}}{\sqrt[n]{P\left(x_{2 k-1}\right)}}+\xi \frac{x_{2 k}}{\sqrt[n]{P\left(x_{2 k}\right)}} .
$$

Segue-se que $Z=\left[\left\{y_{k}: k \in \mathbb{N}\right\}\right]$ é um subespaço de dimensão infinita e em virtude da relação acima, para cada $u=a_{1} y_{k_{1}}+\ldots+a_{r} y_{k_{r}} \in Z$,

$$
\begin{aligned}
P\left(\sum_{i=1}^{r} a_{i} y_{k_{i}}\right) & =P\left(\sum_{i=1}^{r} a_{i} \frac{x_{2 k_{i}-1}}{\sqrt[n]{P\left(x_{2 k_{i}-1}\right)}}+\sum_{i=1}^{r} a_{i} \xi \frac{x_{2 k_{i}}}{\sqrt[n]{P\left(x_{2 k_{i}}\right)}}\right) \\
& =\sum_{i=1}^{r} a_{i}^{n} \frac{P\left(x_{2 k_{i}-1}\right)}{P\left(x_{2 k_{i}-1}\right)}-\sum_{i=1}^{r} a_{i}^{n} \frac{P\left(x_{2 k_{i}}\right)}{P\left(x_{2 k_{i}}\right)}=0 .
\end{aligned}
$$


Corolário 3.1.2. Seja $P \in \mathcal{P}(X)$, onde $X$ é um espaço de Banach complexo de dimensão infinita. Se $P(0)=0$ então $P^{-1}(0)$ contém um subespaço de dimensão infinita.

Demonstração. Seja $P=\sum_{k=1}^{n} P_{k}$ onde $P_{k} \in \mathcal{P}\left({ }^{k} X\right)$ para todo $1 \leq k \leq n$.

De acordo com o teorema 3.1.1, deve existir um subespaço de dimensão infinita $Y_{1} \subset$ $P_{1}^{-1}(0)$. Restringindo $P_{2}$ ao subespaço $Y_{1}$, novamente por 3.1.1, deve existir um subespaço de dimensão infinita $Y_{2} \subset P_{2}^{-1}(0) \cap Y_{1}$. Continuando esse processo obtemos um subespaço de dimensão infinita, $Y_{n}$, satisfazendo

$$
Y_{n} \subset P_{n}^{-1}(0) \cap Y_{n-1} \subset P_{n}^{-1}(0) \cap P_{n-1}^{-1}(0) \cap Y_{n-2} \subset \ldots \subset P_{n}^{-1}(0) \cap \ldots \cap P_{1}^{-1}(0) .
$$

Então $Z:=Y_{n}$, é um subespaço de dimensão infinita contido em $P^{-1}(0)$.

Estudaremos agora zeros de polinômios sob o ponto de vista de J. Ferrer em [11].

Para cada polinômio $P \in \mathcal{P}\left({ }^{n} X\right)$, demonstra-se facilmente que ker $\partial^{n-1} P$ é um subespaço fechado contido em $P^{-1}(0)$. Vale também, o seguinte resultado.

Proposição 3.1.3. Seja $P \in \mathcal{P}\left({ }^{n} X\right)$. Para todo subespaço maximal $Z \subset P^{-1}(0)$ temos ker $\partial^{n-1} P \subseteq Z$.

Demonstração. Seja $Z \subset P^{-1}(0)$ um subespaço maximal. De acordo com a Fórmula de Polarização 2.2.7, se $\partial^{n-1} P(x) \equiv 0$ então $\widehat{\partial^{n-1} P(x)} \equiv 0$. Então, para cada $x \in \operatorname{ker} \partial^{n-1} P$ e $y \in Z$,

$$
\begin{aligned}
P(x+y) & =P(x)+P(y)+\sum_{k=1}^{n-1}\left(\begin{array}{l}
n \\
k
\end{array}\right) \widehat{P} x^{n-k} y^{k} \\
& \left.=\frac{1}{n !} \partial^{n-1} P(x)(x)+\sum_{k=1}^{n-1} \frac{1}{k !(n-k) !} \partial^{n-1 P(x}\right)(\overbrace{x, \ldots, x}^{n-k-1}, \overbrace{y, \ldots, y}^{k}) \\
& =0 .
\end{aligned}
$$

Portanto $Z+$ ker $\partial^{n-1} P$ é um subespaço contido em $P^{-1}(0)$. A maximalidade de $Z$ implica ker $\partial^{n-1} P \subseteq Z$.

Em seu artigo [11], J. Ferrer procura inicialmente estabelecer condições sobre $P$ e $X$ de forma que ker $\partial^{n-1} P$ seja de dimensão infinita. 
Utilizando-se as proposições 2.3 .9 e 1.2 .15 demonstra-se a relação

$$
\overline{[\{\partial P(X)\}]}^{w^{*}}=\left(\partial P(X)_{\perp}\right)^{\perp}=\left(\operatorname{ker} \partial^{n-1} P\right)^{\perp} .
$$

De acordo com esta relação é, grosso modo, razoável esperar que quanto menor for $\partial P(X)$ maior será ker $\partial^{n-1} P$, em particular segue um interessante resultado.

Proposição 3.1.4. Seja $P \in \mathcal{P}\left({ }^{n} X\right)$ onde $X$ é um espaço de Banach. Se $\partial P(X)$ é separável então $\left(\text { ker } \partial^{n-1} P\right)^{\perp}$ é $w^{*}$-separável em $X^{*}$.

Demonstração. Como $\partial P(X)$ é separável em $X^{*}$, o espaço $[\{\partial P(X)\}]$ é separável em $X^{*}$ e portanto existe $\mathcal{D}=\left\{\varphi_{n}: n \in \mathbb{N}\right\} \subset[\{\partial P(X)\}]$ satisfazendo $[\{\partial P(X)\}] \subseteq \overline{\mathcal{D}}$. Como todo aberto na topologia $w^{*}$ de $X^{*}$ é aberto na topologia da norma de $X^{*}$, temos $[\{\partial P(X)\}] \subseteq$

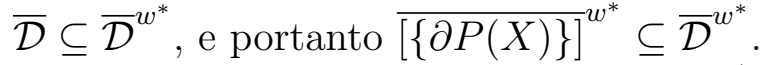

Como claramente $\overline{\mathcal{D}}^{w^{*}} \subseteq \overline{[\{\partial P(X)\}]}{ }^{w^{*}}$, concluímos

$$
\overline{[\{\partial P(X)\}]}]^{w^{*}}=\overline{\mathcal{D}}^{w^{*}} .
$$

De acordo com a relação (3.1), podemos escrever

$$
\overline{\mathcal{D}}^{w^{*}}=\overline{[\{\partial P(X)\}]^{w^{*}}}=\left(\partial P(X)_{\perp}\right)^{\perp}=\left(\operatorname{ker} \partial^{n-1} P\right)^{\perp},
$$

concluindo a demonstração.

Demonstra-se também, que para cada $P \in \mathcal{P}\left({ }^{n} X\right),\left(\operatorname{ker} \partial^{n-1} P\right)^{\perp} \cong\left(X / \text { ker } \partial^{n-1} P\right)^{*}$, portanto, de acordo com a proposição acima, se para algum $P \in \mathcal{P}\left({ }^{n} X\right)$ o conjunto $\partial P(X)$ for separável então $\left(X / \operatorname{ker} \partial^{n-1} P\right)^{*}$ é $w^{*}$-separável. Este fato motivou J. Ferrer a estudar os espaços de dual $w^{*}$-separável. Estudaremos tais espaços na próxima seção.

Nosso interesse agora passa a ser polinômios contínuos sobre espaços de Banach reais, portanto, a partir de agora $X$ denotará sempre um espaço de Banach real. Devemos ressaltar que alguns resultados e definições podem ser estendidos e aplicados em espaços de Banach complexos.

\subsection{Espaços de Dual $w^{*}$-Separável}

Nesta seção, introduziremos uma classe de espaços de Banach reais, que nos permitirá encontrar condições, sob as quais, um determinado tipo de polinômio se anulará em um 
subespaço de dimensão infinita.

Definição 3.2.1. Dizemos que um espaço de Banach $X$ é de classe $\mathcal{W}$, ou simplesmente $X \in \mathcal{W}$ se $X^{*}$ for $w^{*}$-separável.

A proposição a seguir nos permite obter alguns exemplos de espaços de classe $\mathcal{W}$.

Proposição 3.2.2. Se $X$ é separável então $X$ e $X^{*}$ são de classe $\mathcal{W}$.

Demonstração. Verificaremos que $X^{*}$ e $X^{* *}$ são $w^{*}$-separáveis. Para demonstrarmos que $X^{*}$ é $w^{*}$-separável, observemos que de acordo com proposição 1.2.18, a separabilidade de $X$ implica $B_{X^{*}} w^{*}$-metrizável. De acordo com o teorema de Alaoglu 1.2.16, a bola unitária $B_{X^{*}}$ é também $w^{*}$-compacta. Portanto, sob a topologia fraca*, o conjunto $B_{X^{*}}$ é um espaço métrico compacto, consequentemente separável. Como claramente

$$
X^{*}=\bigcup_{k \in \mathbb{N}} k B_{X^{*}},
$$

temos $X^{*} w^{*}$-separável. Portanto $X \in \mathcal{W}$.

Fixemos agora $\mathcal{D}$, um subconjunto denso e enumerável de $X$. Se $\mathcal{I}: X \rightarrow X^{* *}$ for aplicação canônica, em virtude da proposição 1.2.11,

$$
\mathcal{I}(X)=\mathcal{I}(\overline{\mathcal{D}}) \subseteq \overline{\mathcal{I}(\mathcal{D})}^{w^{*}} .
$$

De acordo com o teorema de Goldstine 1.2.17, o conjunto $\mathcal{I}(X)$ é $w^{*}$-denso em $X^{* *}$. Então

$$
X^{* *}=\overline{\mathcal{I}(X)}^{w^{*}} \subseteq \overline{\mathcal{I}(\mathcal{D})}^{w^{*}},
$$

e consequentemente $\mathcal{I}(\mathcal{D})$ é um subconjunto $w^{*}$-denso e enumerável de $X^{* *}$. Concluímos que $X^{*} \in \mathcal{W}$.

Recordando-se que $l_{1}^{*} \cong l_{\infty}$, aplicando-se a proposição $3.2 .2 \mathrm{em} l_{1}$, obtemos o seguinte exemplo.

Exemplo 3.2.3. $l_{\infty} \in \mathcal{W}$.

A proposição a seguir nos fornece uma caracterização muito útil para os espaços de classe $\mathcal{W}$.

Proposição 3.2.4. As seguintes condições são equivalentes:

(i) existe uma aplicação injetora $T \in \mathcal{L}\left(X ; l_{\infty}\right)$; 
(ii) para todo $1 \leq p \leq \infty$, existe uma aplicação injetora $T_{p} \in \mathcal{L}\left(X ; l_{p}\right)$;

(iii) $X \in \mathcal{W}$.

Demonstração. $(i) \Rightarrow($ ii $)$ Seja $T \in \mathcal{L}\left(X ; l_{\infty}\right)$ injetora. Fixemos $\left\{\pi_{n} \circ T: n \in \mathbb{N}\right\} \subset X^{*}$, onde para cada $n \in \mathbb{N}, \pi_{n} \in l_{\infty}^{*}$ é a projeção definida por

$$
\pi_{n}\left(\left(x_{k}\right)_{k \in \mathbb{N}}\right):=x_{n} .
$$

Claramente, a coleção $\left\{\pi_{n} \circ T: n \in \mathbb{N}\right\}$ é pontualmente limitada. Pelo Princípio da Limitação Uniforme, existe $M>0$ tal que $\sup _{n \in \mathbb{N}}\left\|\pi_{n} \circ T\right\|<M$. Fixado $1 \leq p<\infty$, para cada $x \in X$ e para cada $n \in \mathbb{N}$, temos

$$
\sum_{k=1}^{n}\left|\frac{\pi_{k}(T(x))}{2^{k} M}\right|^{p} \leq \sum_{k=1}^{n}\left|\frac{\|x\| M}{2^{k} M}\right|^{p} \leq\|x\|^{p}\left(\sum_{k=1}^{n}\left(\frac{1}{2^{k}}\right)^{p}\right) .
$$

Decorre que para cada $x \in X$,

$$
\sum_{k=1}^{\infty}\left|\frac{\pi_{k}(T(x))}{2^{k} M}\right|^{p} \leq\left(\frac{1}{2^{p}-1}\right)\|x\|^{p} .
$$

Podemos então, definir uma aplicação $T_{p}: X \rightarrow l_{p}$ por

$$
T_{p}(x):=\left(\frac{\pi_{n} \circ T(x)}{2^{n} M}\right)_{n \in \mathbb{N}} .
$$

A injetividade de $T_{p}$ segue imediatamente da injetividade de $T$ e a continuidade imediatamente da relação (3.2).

(ii) $\Rightarrow($ i $)$ Evidente.

(i) $\Rightarrow$ (iii) De acordo com o exemplo 3.2.3, $l_{\infty} \in \mathcal{W}$. Então existe uma coleção $\left\{\psi_{n}: n \in \mathbb{N}\right\} \subset l_{\infty}^{*}$, satisfazendo

$$
{\overline{\left\{\psi_{n}: n \in \mathbb{N}\right\}}}^{w^{*}}=l_{\infty}^{*}
$$

Seja $T \in \mathcal{L}\left(X ; l_{\infty}\right)$ injetora e fixemos a coleção $\left\{\psi_{n} \circ T: n \in \mathbb{N}\right\} \subset X^{*}$. Claramente

$$
\left\{\psi_{n} \circ T: n \in \mathbb{N}\right\}_{\perp}=\bigcap_{n \in \mathbb{N}} \operatorname{ker} \psi_{n} \circ T .
$$

Seja $x \in \bigcap_{n \in \mathbb{N}} \operatorname{ker} \psi_{n} \circ T$. Se $T(x) \neq 0$, existe, pelo teorema de Hahn-Banach, um funcional $\phi \in l_{\infty}^{*}$ tal que $\phi(T(x)) \neq 0$. Por $\left\{\psi_{n}: n \in \mathbb{N}\right\}$ ser $w^{*}$-denso em $l_{\infty}^{*}$, para cada 
$k \in \mathbb{N}$, temos:

$$
W\left(\phi, T(x), \frac{1}{k}\right) \cap\left\{\psi_{n}: n \in \mathbb{N}\right\} \neq \emptyset .
$$

Isso implica $|\phi(T(x))|<\frac{1}{k}$ para todo $k \in \mathbb{N}$ e consequentemente $\phi(T(x))=0$, uma contradição.

Então $T(x)=0$ e, por $T$ ser injetora, $x=0$. Podemos concluir

$$
\bigcap_{n \in \mathbb{N}} \operatorname{ker} \psi_{n} \circ T=\{0\}
$$

Em virtude do teorema 1.2.15 e da relação (3.3),

$$
\left.\overline{\left[\left\{\psi_{n} \circ T: n \in \mathbb{N}\right\}\right.}\right]^{w^{*}}=\left(\left\{\psi_{n} \circ T: n \in \mathbb{N}\right\}_{\perp}\right)^{\perp}=\left(\bigcap_{n \in \mathbb{N}} \operatorname{ker} \psi_{n} \circ T\right)^{\perp}=\{0\}^{\perp}=X^{*}
$$

Por $\left\{\psi_{n} \circ T: n \in \mathbb{N}\right\}$ ser enumerável, a proposição 1.2 .13 implica que $\left[\left\{\psi_{n} \circ T: n \in \mathbb{N}\right\}\right]$ é $w^{*}$-separável. Decorre que $X^{*}$ é $w^{*}$-separável, ou seja, $X \in \mathcal{W}$.

$($ iii $) \Rightarrow(i)$ Seja $\left\{\psi_{n}: n \in \mathbb{N}\right\} \in X^{*} \backslash\{0\}$ uma coleção enumerável satisfazendo

$$
{\overline{\left\{\psi_{n}: n \in \mathbb{N}\right\}}}^{w^{*}}=X^{*}
$$

Para cada $x \in X,\left(\frac{\psi_{n}(x)}{\left\|\psi_{n}\right\|}\right)_{n \in \mathbb{N}} \in l_{\infty}$. Podemos definir uma aplicação $T: X \rightarrow l_{\infty}$ por

$$
T(x):=\left(\frac{\psi_{n}(x)}{\left\|\psi_{n}\right\|}\right)_{n \in \mathbb{N}} .
$$

Verifica-se sem dificuldade que $T$ é linear e contínua. Para verificar a injetividade, seja $x \in X$ tal que $T(x)=0$. Então $x \in \bigcap_{n \in \mathbb{N}} \operatorname{ker} \psi_{n}$. Se fosse $x \neq 0$, de acordo com o teorema de Hahn-Banach, existiria um funcional $\phi \in X^{*}$ tal que $\phi(x) \neq 0$. Por $\left\{\psi_{n}: n \in \mathbb{N}\right\}$ ser $w^{*}$-denso em $X^{*}$, para cada $k \in \mathbb{N}$, temos

$$
W\left(\phi, x, \frac{1}{k}\right) \cap\left\{\psi_{n}: n \in \mathbb{N}\right\} \neq \emptyset
$$

Então $|\phi(x)|<\frac{1}{k}$ para todo $k \in \mathbb{N}$ e consequentemente $\phi(x)=0$, uma contradição. Isso demonstra que $x=0$, e portanto $T$ é injetora.

Exemplo 3.2.5. Se $K$ é um espaço topológico de Hausdorff, compacto e separável, seja $\left(x_{n}\right)_{n \in \mathbb{N}}$ uma sequência densa em $K$. Para cada $f \in \mathcal{C}(K),\left(f\left(x_{n}\right)\right)_{n \in \mathbb{N}} \in l_{\infty}$. Podemos 
definir $T: \mathcal{C}(K) \rightarrow l_{\infty}$ por

$$
T(f):=\left(f\left(x_{n}\right)\right)_{n \in \mathbb{N}} .
$$

Claramente $T$ é linear e contínua.

Se $T(g)=0$ então $g\left(x_{n}\right)=0$ para todo $n \in \mathbb{N}$. Por $\left(x_{n}\right)_{n \in \mathbb{N}}$ ser um conjunto denso em $K$, decorre $g \equiv 0$, e concluímos que $T$ é injetora.

De acordo com a proposição $3.2 .4, \mathcal{C}(K) \in \mathcal{W}$.

Corolário 3.2.6. Sejam $X, Y$ espaços de Banach tais que $X \in \mathcal{W}$ e exista uma aplicação $S \in \mathcal{L}(Y ; X)$ injetora. Então $Y$ pertence $\mathcal{W}$. Em particular todo subespaço de $X$ pertence a $\mathcal{W}$.

Proposição 3.2.7. Seja $Y$ um subespaço fechado de $X$. Se $Y, X / Y \in \mathcal{W}$ então $X \in \mathcal{W}$.

Demonstração. Em virtude da proposição 3.2.4, existem aplicações $T_{1} \in \mathcal{L}\left(Y ; l_{\infty}\right)$ e $T_{2} \in$ $\mathcal{L}\left(X / Y ; l_{\infty}\right)$ injetoras. Para cada $n \in \mathbb{N}$, consideremos a projeção $\pi_{n}: l_{\infty} \rightarrow \mathbb{R}$, definida por

$$
\pi_{n}\left(\left(x_{k}\right)_{k \in \mathbb{N}}\right):=x_{n} .
$$

Fixemos a coleção $\left\{\pi_{n} \circ T_{1}: n \in \mathbb{N}\right\} \subset Y^{*}$ e para cada $n \in \mathbb{N}$ fixemos $\mathcal{T}_{n} \in X^{*}$ uma extensão de Hahn-Banach para $\pi_{n} \circ T_{1}$.

Definimos uma aplicação $T: X \rightarrow l_{\infty}$ por

$$
T(x):=\left(\lambda_{n}(x)\right)_{n \in \mathbb{N}},
$$

onde

$$
\lambda_{n}(x)= \begin{cases}\mathcal{T}_{\frac{n+1}{2}}(x) & \text { se n for ímpar; } \\ \pi_{\frac{n}{2}}\left(T_{2}(x+Y)\right) & \text { se } \mathrm{n} \text { for par. }\end{cases}
$$

Demonstra-se que, de fato, $T(x) \in l_{\infty}$ para cada $x \in X$ e que $T$ é linear. Demonstra-se também que, para cada $x \in X$,

$$
\|T(x)\| \leq\|x\| \max \left\{\left\|T_{1}\right\|,\left\|T_{2}\right\|\right\},
$$

assegurando a continuidade de $T$.

Se $x \in X$ é tal que $T(x)=0$, então pela definição de $T$, temos $T_{2}(x+Y)=0$ e $\mathcal{T}_{n}(x)=0$ para todo $n \in \mathbb{N}$. Por $T_{2}$ ser injetora, temos $x \in Y$ e então, para cada $n \in \mathbb{N}$,

$$
\pi_{n}\left(T_{1}(x)\right)=\pi_{n} \circ T_{1}(x)=\mathcal{T}_{n}(x)=0,
$$


portanto

$$
T_{1}(x)=0
$$

Da injetividade de $T_{1}$ decorre $x=0$, e concluímos que $T$ é injetora. Concluímos que existe uma aplicação $T \in \mathcal{L}\left(X ; l_{\infty}\right)$ injetora, o que, em virtude de 3.2 .4 , implica $X \in \mathcal{W}$.

Lema 3.2.8. Se $Y$ é um subespaço fechado de um espaço de Banach $X$ então $Y^{\perp}$ é $w^{*}$ separável se, e somente se, existe uma coleção $\left\{\psi_{n}: n \in \mathbb{N}\right\} \subset X^{*}$ satisfazendo

$$
Y=\bigcap_{n \in \mathbb{N}} \operatorname{ker} \psi_{n}
$$

Demonstração. Fixemos uma coleção enumerável $\left\{\psi_{n}: n \in \mathbb{N}\right\} \subset Y^{\perp}$ satisfazendo

$$
\left.Y^{\perp}=\overline{\left[\left\{\psi_{n}: n \in \mathbb{N}\right\}\right.}\right]^{*}
$$

Em virtude da proposição 1.2.15 temos

$$
\overline{\left[\left\{\psi_{n}: n \in \mathbb{N}\right\}\right]^{w^{*}}}=\left(\left\{\psi_{n}: n \in \mathbb{N}\right\}_{\perp}\right)^{\perp}=\left(\bigcap_{n \in \mathbb{N}} \operatorname{ker} \psi_{n}\right)^{\perp}
$$

Provaremos que $Y=\bigcap_{n \in \mathbb{N}} \operatorname{ker} \psi_{n}$.

Para cada $x \in Y$, por $\left\{\psi_{n}: n \in \mathbb{N}\right\} \subset Y^{\perp}$, decorre $x \in \bigcap_{n \in \mathbb{N}}$ ker $\psi_{n}$ e concluímos, $Y \subseteq$ $\bigcap_{n \in \mathbb{N}} \operatorname{ker} \psi_{n}$.

Se existisse $x \in \bigcap_{n \in \mathbb{N}}$ ker $\psi_{n} \backslash Y$, por $Y$ ser fechado, o teorema de Hahn-Banach implicaria a existência de $\phi \in B_{X^{*}}$ satisfazendo $\phi(x) \neq 0$ e $\phi(Y) \subseteq\{0\}$. Então

$$
\phi \in Y^{\perp}=\overline{\left[\left\{\psi_{n}: n \in \mathbb{N}\right\}\right]^{w^{*}}}=\left(\left\{\psi_{n}: n \in \mathbb{N}\right\}_{\perp}\right)^{\perp}=\left(\bigcap_{n \in \mathbb{N}} \operatorname{ker} \psi_{n}\right)^{\perp}
$$

e consequentemente $\phi(x)=0$, uma contradição. Concluímos, $\bigcap_{n \in \mathbb{N}}$ ker $\psi_{n} \subseteq Y$, estabelecendo a igualdade.

Por outro lado, se $Y=\bigcap_{n \in \mathbb{N}} \operatorname{ker} \psi_{n}$ para alguma coleção $\left\{\psi_{n}: n \in \mathbb{N}\right\} \subset X^{*}$, em virtude da propoposição 1.2.15,

$$
\left.Y^{\perp}=\left(\bigcap_{n \in \mathbb{N}} \operatorname{ker} \psi_{n}\right)^{\perp}=\left(\left\{\psi_{n}: n \in \mathbb{N}\right\}_{\perp}\right)^{\perp}=\overline{\left[\left\{\psi_{n}: n \in \mathbb{N}\right\}\right.}\right]^{w^{*}}
$$

demonstrando que $Y^{\perp}$ é $w^{*}$-separável. 
Proposição 3.2.9. Se $\left\{Y_{n}: n \in \mathbb{N}\right\}$ é uma coleção de subespaços fechados de $X$ tal que para cada $n \in \mathbb{N}$ o espaço $Y_{n}^{\perp}$ seja $w^{*}$-separável, então o subespaço $Y:=\bigcap_{n \in \mathbb{N}} Y_{n}$ satisfaz as seguintes condições:

(i) $Y^{\perp}$ é $w^{*}$-separável;

(ii) se $X \notin \mathcal{W}$ então $Y \notin \mathcal{W}$.

Demonstração. Em virtude do lema 3.2.8, para cada $m \in \mathbb{N}$, existe $\left\{\psi_{m n}: n \in \mathbb{N}\right\} \subset X^{*}$, satisfazendo

$$
Y_{m}=\bigcap_{n \in \mathbb{N}} \operatorname{ker} \psi_{m n}
$$

De acordo com a proposição 1.2 .15 , temos

$$
\begin{aligned}
\left(\bigcap_{m \in \mathbb{N}} Y_{m}\right)^{\perp} & =\left(\bigcap_{m, n \in \mathbb{N}} \operatorname{ker} \psi_{m n}\right)^{\perp} \\
& =\left(\left\{\psi_{m n}: m, n \in \mathbb{N}\right\}_{\perp}\right)^{\perp}=\overline{\left[\left\{\psi_{m n}: m, n \in \mathbb{N}\right\}\right]} w^{*},
\end{aligned}
$$

o que verifica $(i)$.

Por $Y$ ser fechado, temos $Y^{\perp} \cong(X / Y)^{*}$ e pelo item $(i), X / Y \in \mathcal{W}$. Se $Y \in \mathcal{W}$, pela proposição 3.2.7 teríamos $X \in \mathcal{W}$ e isso verifica $(i i)$.

\subsection{Zeros de Polinômios Fracamente Contínuos sobre Limitados}

Os resultados desta seção determinam construtivamente subespaços de dimensão infinita contidos em zeros de polinômios fracamente contínuos sobre os subconjuntos limitados de um espaço de Banach não pertencendo a $\mathcal{W}$.

Proposição 3.3.1. Sejam $X$ um espaço de Banach e $P \in \mathcal{P}_{w}\left({ }^{n} X\right)$. Se $X \notin \mathcal{W}$ então ker $\partial^{n-1} P \notin \mathcal{W}$.

Demonstração. De acordo com a proposição 2.4.9, o conjunto $\partial P(X)$ é separável em $X^{*} \mathrm{e}$ em virtude da proposição 3.1.4, o conjunto $\left(\operatorname{ker} \partial^{n-1} P\right)^{\perp}$ é $w^{*}$-separável. Temos

$$
\left(\operatorname{ker} \partial^{n-1} P\right)^{\perp} \cong\left(X / \operatorname{ker} \partial^{n-1} P\right)^{*}
$$


e portanto $X / \operatorname{ker} \partial^{n-1} P \in \mathcal{W}$. Se ker $\partial^{n-1} P \in \mathcal{W}$ teríamos, de acordo com a proposição 3.2.7, $X \in \mathcal{W}$, uma contradição.

Corolário 3.3.2. Se $X \notin \mathcal{W}$ então para cada $P \in \mathcal{P}_{w}\left({ }^{n} X\right)$, qualquer espaço maximal $Z \subset P^{-1}(0)$ não pertence a $\mathcal{W}$.

Demonstração. De acordo com a proposição 3.1.3, dado um polinômio $P \in \mathcal{P}_{w}\left({ }^{n} X\right)$ e um subespaço maximal $Z \in P^{-1}(0)$ temos ker $\partial^{n-1} P \subseteq Z$. De acordo com a proposição 3.3.1 o espaço ker $\partial^{n-1} P \notin \mathcal{W}$ e pelo corolário 3.2.6, $Z \notin \mathcal{W}$.

O resultado a seguir é uma outra caracterização para os espaços de classe $\mathcal{W}$. Recordemos que um polinômio $P \in \mathcal{P}\left({ }^{n} X\right)$ é positivo definido se e somente se $P(x) \geq 0$ para cada $x \in X$ e se $P(x)=0$ então $x=0$.

Corolário 3.3.3. Se $X$ é um espaço de Banach, as seguintes condições são equivalentes:

(i) $X \in \mathcal{W}$.

(ii) Para todo $n$ par, $X$ admite um polinômio positivo definido $P \in \mathcal{P}_{N}\left({ }^{n} X\right)$.

(iii) Para todo $n$ par, $X$ admite um polinômio positivo definido $P \in \mathcal{P}_{w}\left({ }^{n} X\right)$.

(iv) Existe um inteiro par $n$, tal que $X$ admite um polinômio positivo definido $P \in \mathcal{P}_{w}\left({ }^{n} X\right)$.

(v) Existe um inteiro par $n$, tal que $X$ admite um polinômio positivo definido $P \in \mathcal{P}_{N}\left({ }^{n} X\right)$.

Demonstração. (i) $\Rightarrow$ (ii) Se $X \in \mathcal{W}$, pela proposição 3.2.4, existe uma aplicação $T \in$ $\mathcal{L}\left(X ; l_{2}\right)$ injetora. Se $\left\{e_{k}: k \in \mathbb{N}\right\}$ for a base canônica de $l_{2}$, para cada $n$ par, definimos

$$
P(x):=\sum_{k=1}^{\infty} 2^{-k}\left\langle e_{k}, T x\right\rangle^{n} .
$$

Claramente $P \in \mathcal{P}_{N}\left({ }^{n} X\right)$ e $P(x) \geq 0$ para todo $x \in X$. Se $P(x)=0$ então $\left\langle e_{k}, T x\right\rangle=0$ para todo $k \in \mathbb{N}$ e consequentemente $T x=0$. Por $T$ ser injetora, $x=0$.

(ii) $\Rightarrow$ (iii) De acordo com a proposição 2.4.5 temos $\mathcal{P}_{N}\left({ }^{n} X\right) \subseteq \mathcal{P}_{w}\left({ }^{n} X\right)$. A conclusão é evidente.

$($ iii $) \Rightarrow(i v)$ Evidente.

$(i v) \Rightarrow(v)$ Sob a condição $(i v)$, devemos ter $X \in \mathcal{W}$, pois do contrário, de acordo com o corolário 3.3.2, nenhum polinômio em $\mathcal{P}_{w}\left({ }^{m} X\right)$ pode ser positivo definido. A tese decorre de $(i) \Rightarrow(i i)$. 
$(v) \Rightarrow(i)$ Analogamente ao caso $(i v) \Rightarrow(v)$, o corolário 3.3.2 implica $X \in \mathcal{W}$.

Corolário 3.3.4. Se $X$ é um espaço de Banach real de dimensão infinita, ou $X$ admite um polinômio 2-homogêneo nuclear positivo definido ou para todo $P \in \mathcal{P}_{w}\left({ }^{n} X\right)$ existe um subespaço fechado $Z \subset P^{-1}(0)$ tal que $Z \notin \mathcal{W}$.

Demonstração. Se $X$ não adimitir um polinômio 2-homogêneo nuclear positivo definido, então pelo corolário 3.3.3 temos $X \notin \mathcal{W}$.

De acordo com a proposição 3.3.1, para todo $P \in \mathcal{P}_{w}\left({ }^{n} X\right)$ temos ker $\partial^{n-1} P \notin \mathcal{W}$ que é um subespaço fechado contido $P^{-1}(0)$.

Proposição 3.3.5. Seja $\left\{P_{k}: k \in \mathbb{N}\right\}$ uma coleção de polinômios homogêneos tais que para cada $k, P_{k} \in \mathcal{P}_{w}\left({ }^{n_{k}} X\right)$. Se $X \notin \mathcal{W}$ então $X$ admite um subespaço fechado $Z \subset \bigcap_{k \in \mathbb{N}} P_{k}^{-1}(0)$ e tal que $Z \notin \mathcal{W}$.

Demonstração. De acordo com a proposição 2.4.9, para cada $k \in \mathbb{N}$, o conjunto $\partial P_{k}(X)$ é separável em $X^{*}$. Então, de acordo com a proposição 3.1.4, para cada $k \in \mathbb{N}$, o conjunto $\left(\text { ker } \partial^{n_{k}-1} P_{k}\right)^{\perp}$ é $w^{*}$-separável. Seja $Z \subset X$ definido por

$$
Z:=\bigcap_{k \in \mathbb{N}} \operatorname{ker} \partial^{n_{k}-1} P_{k}
$$

De acordo com a proposição 3.2 .9 , o conjunto $Z^{\perp}$ também é $w^{*}$-separável, e por $X \notin \mathcal{W}$, temos $Z \notin \mathcal{W}$. Em virtude de ker $\partial^{n_{k}-1} P_{k} \subseteq P_{k}^{-1}(0)$, para cada $k \in \mathbb{N}$, temos

$$
Z \subset \bigcap_{k \in \mathbb{N}} P_{k}^{-1}(0)
$$

Proposição 3.3.6. Sejam $X, Y$ espaços de Banach com $X \notin \mathcal{W}$. Seja $\left\{P_{k}: k \in \mathbb{N}\right\}$ uma coleção de polinômios tais que para cada $k \in \mathbb{N}, P_{k} \in \mathcal{P}_{w}\left({ }^{n_{k}} X ; Y\right)$. Então existe um subespaço fechado $Z \subset \bigcap_{k \in \mathbb{N}} P_{k}^{-1}(0)$ e tal que $Z \notin \mathcal{W}$.

Demonstração. De acordo com com a proposição 2.4.9, para cada $k \in \mathbb{N}$, o conjunto $P_{k}(X)$ é separável em $Y$. Definimos

$$
Y_{0}=\overline{\left[\left\{\bigcup_{k \in \mathbb{N}} P_{k}(X)\right\}\right]} .
$$

Claramente $Y_{0}$ é um espaço de Banach separável. 
De acordo com 3.2.2, $Y_{0} \in \mathcal{W}$, e em virtude do corolário 3.3.3, $Y_{0}$ admite um polinômio 2-homogêneo nuclear positivo definido

$$
P:=\sum_{k=1}^{\infty} a_{n} \phi_{k}^{2}
$$

onde $\left\{\phi_{n}: n \in \mathbb{N}\right\}$ é uma coleção limitada em $Y_{0}^{*}$ e $\left(a_{n}\right)_{n \in \mathbb{N}} \in l_{1}$.

Para cada $n \in \mathbb{N}$ seja $\Phi_{k} \in Y^{*}$ uma extensão de Hahn-Banach de $\phi_{k}$.

Claramente

$$
\left(\bigcap_{k \in \mathbb{N}} \operatorname{ker} \Phi_{k}\right) \cap Y_{0}=\{0\} .
$$

Para cada $k, r \in \mathbb{N}$ definimos $Q_{k r} \in \mathcal{P}_{w}\left({ }^{n_{k}} X\right)$ por

$$
Q_{k r}(x):=\Phi_{r}\left(P_{k}(x)\right)
$$

Analogamente à demonstração da proposição 3.3.5, para todo $k, r \in \mathbb{N}$ o conjunto $\left(\operatorname{ker} \partial^{n_{k}-1} Q_{k r}\right)^{\perp}$ é $w^{*}$-séparável. Definindo-se $Z:=\bigcap_{r, k \in \mathbb{N}} \operatorname{ker} \partial^{n_{k}-1} Q_{k r}$, o item $(i)$ da proposição 3.2.9, implica $Z^{\perp} w^{*}$-separável e por $X \notin \mathcal{W}$, o item (ii) da mesma proposição implica $Z \notin \mathcal{W}$.

Em virtude de ker $\partial^{n_{k}-1} Q_{k r} \subseteq Q_{k r}^{-1}(0), k, r \in \mathbb{N}$, para cada $x \in Z$,

$$
\Phi_{r}\left(P_{k}(x)\right)=Q_{k r}(x)=0, k, r \in \mathbb{N} .
$$

Decorre que, para cada $k \in \mathbb{N}$,

$$
P_{k}(x) \in\left(\bigcap_{r \in \mathbb{N}} \operatorname{ker} \Phi_{r}\right) \cap Y_{0}
$$

e então, pela relação $(3.4), P_{k}(x)=0$ para todo $k \in \mathbb{N}$.

Como assinalado por J. Ferrer em [11], notando-se que vizinhanças fracas de zero contém subespaços de codimensão finita, é possível obter um grande generalização para o resultado anterior.

Lema 3.3.7. Em um espaço de Banach $X$, toda vizinhança fraca de zero contém algum subespaço fechado $Z$ tal que $Z^{\perp}$ é $w^{*}$-separável.

Demonstração. Se $U$ é uma vizinhança fraca de 0 então existem $\phi_{1}, \ldots, \phi_{n} \in X^{*}$ e $\epsilon>0$ tais 
que

$$
\bigcap_{k=1}^{n} \operatorname{ker} \phi_{k} \subset W\left(0, \phi_{1}, \ldots, \phi_{n}, \epsilon\right) \subset U .
$$

Definindo $Z:=\bigcap_{k=1}^{n} \operatorname{ker} \phi_{k}$, o lema 3.2.8 implica $Z^{\perp} w^{*}$-separável.

Teorema 3.3.8. Sejam $X, Y$ espaços de Banach e $f: X \rightarrow Y$ uma aplicação fracamente contínua sobre os subconjuntos limitados de $X$. Se $X \notin \mathcal{W}$ então existe um subespaço fechado $Z \subset f^{-1}(0)$, tal que $Z \notin \mathcal{W}$.

Demonstração. Sejam $B_{X}$ e $B_{Y}$ respectivamente as bolas unitárias fechadas de $X$ e $Y$. Para cada $m, n \in \mathbb{N}$, em virtude das hipóteses sobre $f$, o conjunto $f^{-1}\left(\frac{1}{m} B_{Y}\right) \cap n B_{X}$ é uma vizinhança fraca de zero em $n B_{X}$, e portanto, de acordo com o lema anterior, existe um subespaço fechado $Z_{m n}$ tal que $Z_{m n}^{\perp}$ é $w^{*}$-separável e

$$
Z_{m n} \cap n B_{X} \subseteq f^{-1}\left(\frac{1}{m} B_{Y}\right) \cap n B_{X} .
$$

De acordo com o item $(i)$ da proposição 3.2.9, definindo-se $Z:=\bigcap_{m, n \in \mathbb{N}} Z_{m n}$, temos $Z^{\perp}$ $w^{*}$-separável. Como $X \notin \mathcal{W}$, o item (ii) da mesma proposição implica $Z \notin \mathcal{W}$. Claramente, se $x \in Z$ então $f(x) \in \bigcap_{m \in \mathbb{N}} \frac{1}{m} B_{Y}$, ou seja, $f(x)=0$.

Corolário 3.3.9. Dados espaços de Banach $X, Y$ com $X \notin \mathcal{W}$, seja uma sequência $\left(f_{k}\right)_{k \in \mathbb{N}}$ de funções de $X$ em $Y$, fracamente contínuas sobre os limitados de $X$. Se $f_{n}(0)=0$ para todo $n \in \mathbb{N}$ e para todo $x \in X$ existir o limite

$$
f(x):=\lim _{n \rightarrow \infty} f_{n}(x)
$$

então existe um subespaço fechado $Z \subset f^{-1}(0)$ tal que $Z \notin \mathcal{W}$.

Demonstração. Em virtude do teorema 3.3.8, para cada $n \in \mathbb{N}$ existe um subespaço fechado $Z_{n} \subset f_{n}^{-1}(0)$, tal que $Z_{n}^{\perp}$ seja $w^{*}$-separável.

De acordo com o item $(i)$ da proposição 3.2 .9 , definindo-se $Z:=\bigcap_{n \in \mathbb{N}} Z_{n}$, temos $Z^{\perp}$ $w^{*}$-separável. Por $X \notin \mathcal{W}$, o item $(i i)$ da mesma proposição implica $Z \notin \mathcal{W}$. Claramente, se $x \in Z$ então $f_{n}(x)=0$ para todo $n \in \mathbb{N}$ e portanto $f(x)=\lim _{n \rightarrow \infty} f_{n}(x)=0$. 


\subsection{Zeros de Polinômios sobre Espaços com a Propriedade DP}

Com base nos resultados de J. Ferrer apresentados nas seções anteriores, estudaremos algumas condições, sob as quais todo polinômio $n$-homogêneo contínuo se anule em um subespaço de dimensão infinita. Estudaremos tais resultados sobre a classe dos espaços com a propriedade Dunford-Pettis. Para um estudo detalhado sobre espaços com a propriedade de Dunford-Pettis, recomendamos [9] e [14].

Definição 3.4.1. Dizemos que um espaço de Banach $X$ tem a propriedade de DunfordPettis, ou propriedade $D P$, se para quaisquer sequências $\left(x_{n}\right)_{n \in \mathbb{N}}$ em $X$ e $\left(\varphi_{n}\right)_{n \in \mathbb{N}}$ em $X^{*}$, convergindo fracamente a zero, a sequência $\left(\varphi_{n}\left(x_{n}\right)\right)_{n \in \mathbb{N}}$ converge a zero.

A demonstração da proposição a seguir pode ser encontrada com todos os detalhes em [9](pág. 115).

Proposição 3.4.2. Se $X$ tem a propriedade de Dunford-Pettis então $\mathcal{P}\left({ }^{n} X\right)=\mathcal{P}_{\text {wsc }}\left({ }^{n} X\right)$.

Corolário 3.4.3. Seja $X$ um espaço com a propriedade de Dunford-Pettis não contendo cópia de $l_{1}$, então $\mathcal{P}_{w}\left({ }^{n} X\right)=\mathcal{P}\left({ }^{n} X\right)$.

Demonstração. De acordo com a proposição 3.4.2, se $X$ tem a propriedade de DunfordPettis, então $\mathcal{P}\left({ }^{n} X\right)=\mathcal{P}_{\text {wsc }}\left({ }^{n} X\right)$. De acordo com a proposição 2.4.6, se $X$ não contém cópia de $l_{1}$, então $\mathcal{P}_{w s c}\left({ }^{n} X\right)=\mathcal{P}_{w}\left({ }^{n} X\right)$.

Utilizando-se o corolário 3.4.3 acima, e os resultados estudados nas seções 3.2 e 3.3, obtemos um interessante corolário.

Corolário 3.4.4. Seja $X$ um espaço com a propriedade de Dunford-Pettis, com $X \notin \mathcal{W}$ e não contendo cópia de $l_{1}$. Então todo polinômio em $\mathcal{P}\left({ }^{n} X\right)$ se anula em um subespaço de dimensão infinita.

Nosso próximo objetivo é exibir um espaço de Banach real $X$ satisfazendo as hipóteses deste corolário, ou seja

- $X \notin \mathcal{W}$

- $X$ não contém cópia de $l_{1}$;

- $X$ tem a propriedade de Dunford-Pettis. 
O próximo teorema será útil na procura por espaços satisfazendo essas condições. Esse resultado foi publicado originalmente em [21] e sua demonstração pode ser encontrada com todos os detalhes em [21] ou [14].

Teorema 3.4.5. (Pethe e Thakare) Seja $X$ um espaço de Banach. Então $X^{*}$ tem a propriedade Schur se e somente se $X$ tem a propriedade de Dunford-Pettis e não contém cópia de $l_{1}$.

Vimos no capítulo 1, seção 1.4 , que para qualquer $\Gamma$ infinito, $l_{1}(\Gamma)$ tem a propriedade de Schur. De acordo com o teorema 3.4.5 acima, lembrando-se que $\left(c_{0}(\Gamma)\right)^{*} \cong l_{1}(\Gamma)$, concluímos que $c_{0}(\Gamma)$ tem a propriedade de Dunford-Pettis e não contém cópia de $l_{1}$. Temos também o seguinte resultado.

Proposição 3.4.6. Se $\Gamma$ é um conjunto não enumerável então $c_{0}(\Gamma) \notin \mathcal{W}$.

Demonstração. Sejam $\mathcal{B}=\left\{e_{\lambda}: \lambda \in \Gamma\right\}$ a base canônica de $c_{0}(\Gamma)$ e $T \in \mathcal{L}\left(c_{0}(\Gamma) ; l_{2}\right)$ uma aplicação linear arbitrária. Para cada $n \in \mathbb{N}$ definimos

$$
\Gamma_{n}:=\left\{\lambda \in \Gamma:\left\|T e_{\lambda}\right\| \geq \frac{1}{n}\right\}
$$

Se para algum $n_{0}$ o conjunto $\Gamma_{n_{0}}$ for infinito, podemos fixar um conjunto infinito enumerável $\left\{\lambda_{k}: k \in \mathbb{N}\right\} \subset \Gamma_{n_{0}}$ e consequentemente $\left(e_{\lambda_{k}}\right)_{k \in \mathbb{N}}$ é uma sequência convergindo fracamente a zero.

Se $\langle.,\rangle:. l_{2} \times l_{2} \rightarrow \mathbb{R}$ é o produto interno usual de $l_{2}$, fixemos o polinômio $P$, definido por

$$
P(x):=\langle T(x), T(x)\rangle, x \in c_{0}(\Gamma) .
$$

Claramente $P \in \mathcal{P}\left({ }^{2} c_{0}(\Gamma)\right)$.

Por $c_{0}(\Gamma)$ ter a propriedade de Dunford-Pettis, o teorema 3.4.2 implica

$$
\mathcal{P}\left({ }^{2} c_{0}(\Gamma)\right)=\mathcal{P}_{w s c}\left({ }^{2} c_{0}(\Gamma)\right)
$$

portanto

$$
\lim _{k \rightarrow \infty} P\left(e_{\lambda_{k}}\right)=0
$$

Consequentemente existe $k_{0} \in \mathbb{N}$ tal que

$$
P\left(e_{\lambda_{k}}\right)^{\frac{1}{2}}=\left\|T e_{\lambda_{k}}\right\|<\frac{1}{n_{0}}
$$

sempre que $k \geq k_{0}$, uma contradição. 
Podemos então concluir que $\Gamma_{n}$ é finito para todo $n \in \mathbb{N}$, e portanto

$$
\left\{\lambda \in \Gamma: T e_{\lambda} \neq 0\right\}=\bigcup_{n \in \mathbb{N}} \Gamma_{n}
$$

é enumerável.

Por $\mathcal{B}=\left\{e_{\lambda}: \lambda \in \Gamma\right\}$ não ser enumerável, deve existir $\lambda \in \Gamma$ tal que $T e_{\lambda}=0$ e portanto a aplicação $T$ não pode ser injetora.

Concluímos que não existe aplicação linear injetora $T \in \mathcal{L}\left(c_{0}(\Gamma) ; l_{2}\right)$. Segundo a proposição 3.2.4, $c_{0}(\Gamma) \notin \mathcal{W}$.

Concluímos que se $\Gamma$ for um conjunto não enumerável, então $c_{0}(\Gamma)$ é um espaço de Banach com a propriedade de Dunford-Pettis, não contendo cópia de $l_{1}$ e não pertencendo a $\mathcal{W}$. De acordo com nosso estudo, fica estabelecido que $\mathcal{P}\left({ }^{n} c_{0}(\Gamma)\right)=\mathcal{P}_{w}\left({ }^{n} c_{0}(\Gamma)\right)$. Concluímos que todo polinômio $n$-homogêneo contínuo definido sobre $c_{0}(\Gamma)$, se anula em um subespaço de dimensão infinita. Este fato foi verificado por J. Ferrer em [12], e neste mesmo artigo obteve o seguinte resultado.

Proposição 3.4.7. Seja $P \in \mathcal{P}\left({ }^{n} c_{0}(\Gamma)\right)$, onde $\Gamma$ é um conjunto não enumerável. Então ker $\partial^{n-1} P$ contém um subespaço isometricamente isomorfo a $c_{0}(\Gamma)$.

Demonstração. Se $\left\{e_{\lambda}: \lambda \in \Gamma\right\}$ é a base canônica de $c_{0}(\Gamma)$, para cada $n \in \mathbb{N}$ definimos

$$
\Gamma_{n}:=\left\{\lambda \in \Gamma:\left\|\partial^{n-1} P\left(e_{\lambda}\right)\right\| \geq \frac{1}{n}\right\}
$$

Se para algum $n_{0}$ o conjunto $\Gamma_{n_{0}}$ for infinito, podemos fixar um conjunto infinito enumerável $\left\{\lambda_{k}: k \in \mathbb{N}\right\} \subset \Gamma_{n_{0}}$ e consequentemente $\left(e_{\lambda_{k}}\right)_{k \in \mathbb{N}}$ é uma sequência convergindo fracamente a zero.

De acordo com o que discutimos acima $\mathcal{P}\left({ }^{n} c_{0}(\Gamma)\right)=\mathcal{P}_{w}\left({ }^{n} c_{0}(\Gamma)\right)$, e em virtude da proposição 2.4.8, a aplicação $\partial^{n-1} P$ é fracamente contínua sobre os subconjuntos limitados de $c_{0}(\Gamma)$. Consequentemente

$$
\lim _{k \rightarrow \infty}\left\|\partial^{n-1} P\left(e_{k}\right)\right\|=0
$$

uma contradição.

Então, para cada $n \in \mathbb{N}, \Gamma_{n}$ é finito. Concluímos que

$$
\Gamma_{0}:=\left\{\lambda \in \Gamma: \partial P^{n-1}\left(e_{\lambda}\right) \neq 0\right\}=\bigcup_{n \in \mathbb{N}} \Gamma_{n}
$$


é um conjunto enumerável, e portanto existe uma bijeção entre $\Gamma$ e $\Gamma \backslash \Gamma_{0}$.

Definindo-se

$$
Y:=\overline{\left[\left\{e_{\lambda}: \lambda \in \Gamma \backslash \Gamma_{0}\right\}\right]},
$$

verifica-se que $Y \subset \operatorname{ker} \partial^{n-1} P$ e $Y \cong c_{0}(\Gamma)$.

Em virtude de ker $\partial^{n-1} P \subseteq P^{-1}(0)$ para todo $P \in \mathcal{P}\left({ }^{n} c_{0}(\Gamma)\right)$, concluímos que, se $\Gamma$ for um conjunto não enumerável, todo polinômio $n$-homogêneo definido sobre $c_{0}(\Gamma)$ se anula em um subespaço isometricamente isomorfo a $c_{0}(\Gamma)$.

Corolário 3.4.8. Seja $\left\{P_{k}: k \in \mathbb{N}\right\}$ uma coleção de polinômios homogêneos tais que $P \in$ $\mathcal{P}\left({ }^{n_{k}} c_{0}(\Gamma)\right)$, onde $\Gamma$ é um conjunto não enumerável. Então existe um subsepaço $Z$, tal que $Z \subset \bigcap_{k \in \mathbb{N}} P_{k}^{-1}(0)$ e $Z \cong c_{0}(\Gamma)$.

Demonstração. Procedendo-se como na proposição 3.4.7, para cada $k \in \mathbb{N}$ o conjunto $\Gamma_{k}:=\left\{\lambda \in \Gamma: \partial^{n_{k}-1} P_{k}\left(e_{\lambda}\right) \neq 0\right\}$ é enumerável, consequentemente o conjunto $\Gamma_{0}:=\bigcup_{k \in \mathbb{N}} \Gamma_{k}$ é enumerável. Definindo-se

$$
Z:=\overline{\left[\left\{e_{\lambda}: \lambda \in \Gamma / \Gamma_{0}\right\}\right]},
$$

demonstra-se sem dificuldade que $Z \subset \bigcap_{k \in \mathbb{N}} P_{k}^{-1}(0)$ e $Z \cong c_{0}(\Gamma)$.

Os resultados a seguir estabelecem que se $K$ é um espaço compacto não satisfazendo a condição de cadeia contável ou $C C C$, então todo polinômio $n$-homogêneo definido sobre $\mathcal{C}(K)$, se anula em um subespaço isometricamente isomorfo a $c_{0}(\Gamma)$, para algum $\Gamma$ não enumerável. Estes resultados podem ser encontrados com todos os detalhes em [12].

Definição 3.4.9. Dizemos que um espaço topológico satisfaz a condição de cadeia contável ou $C C C$ se qualquer coleção de abertos dois a dois disjuntos for enumerável.

Seja $\Omega$ é um espaço topológico completamente regular, ou seja, um espaço onde os subconjuntos unitários são fechados e para cada $x \in \Omega$ e cada fechado $F$ não contendo $x$ existe uma função real $f$, contínua, tal que $f(x)=1$ e $f(F) \subseteq\{0\}$. Se $\mathcal{C}(\Omega)$ é o conjunto das aplicações contínuas limitadas reais definidas em $\Omega$, seja $\phi: \Omega \rightarrow B_{\mathcal{C}(\Omega)^{*}}$ definida por

$$
\phi(t)(f):=\phi_{t}(f)=f(t), t \in \Omega, f \in \mathcal{C}(\Omega) .
$$

Considerando-se sobre $\phi(\Omega)$ a topologia fraca* induzida de $\mathcal{C}(\Omega)^{*}$, verifica-se facilmente que $\phi: \Omega \rightarrow \phi(\Omega)$ é contínua, e por $\Omega$ ser completamente regular, $\phi$ é injetora. Se $V \subseteq \Omega$ 
é um aberto arbitrário, para cada $t \in V$, por $\Omega$ ser completamente regular, existe $g \in \mathcal{C}(\Omega)$ satisfazendo

$$
g(\Omega \backslash V) \subseteq\{0\} \quad \text { e } g(t)=1
$$

Então $W\left(\phi(t), g, \frac{1}{2}\right) \cap \phi(\Omega)$ é uma vizinhança aberta de $\phi(t)$, contida em $\phi(V)$ e concluímos que $\phi(V)$ é aberto em $\phi(\Omega)$. Por $V$ ser um aberto arbitrário de $\Omega$, concluímos que $\phi$ é uma aplicação aberta.

Segue-se que, sob a topologia fraca*, $\phi: \Omega \rightarrow \phi(\Omega)$ é um homeomorfismo e portanto $B_{\mathcal{C}(\Omega)^{*}}$ contém um subespaço homeomorfo a $\Omega$. Por simplicidade identificaremos $\Omega$ com $\phi(\Omega)$ de modo a omitir referências à função $\phi$. Diremos então que $B_{\mathcal{C}(\Omega)^{*}}$ contém $\Omega$.

Definimos $\beta \Omega$, a compactificação de Stone-Čech de $\Omega$, por

$$
\beta \Omega:=\bar{\Omega}^{w^{*}} .
$$

Em virtude do teorema de Alaoglu 1.2.16, o conjunto $B_{\mathcal{C}(\Omega)^{*}}$ é $w^{*}$-compacto. Consequentemente $\beta \Omega$ é $w^{*}$-compacto. Demonstra-se que para cada $f \in \mathcal{C}(\Omega)$ existe uma única $F \in \mathcal{C}(\beta \Omega)$ satisfazendo

$$
F \uparrow_{\Omega} \equiv f \quad \text { e }\|F\|=\|f\| \text {. }
$$

Para mais detalhes sobre compactificação de Stone-Čech, recomendamos [13] e [24].

Exemplo 3.4.10. Verificaremos que o espaço compacto $\beta \mathbb{N} \backslash \mathbb{N}$ não satisfaz a $C C C$.

Seja $\left\{q_{n}: n \in \mathbb{N}\right\}$ uma enumeração dos racionais em $\mathbb{R}$ e para cada $x \in \mathbb{R} \backslash \mathbb{Q}$ fixemos uma sequência de racionais $\left(q_{n_{k}}\right)_{k \in \mathbb{N}}$ convergindo a $x$. Seja $N_{x}:=\left\{n_{k}: k \in \mathbb{N}\right\}$ o conjunto dos índices dessa sequência. Observemos que se $x, y \in \mathbb{R} \backslash \mathbb{Q}$ são distintos, então $N_{x} \cap N_{y}$ é finito, pois duas sequências convergindo para reais distintos coincidem no máximo em um número finito de termos.

Para cada $x \in \mathbb{R} \backslash \mathbb{Q}$ definimos a aplicação $f_{x}: \mathbb{N} \rightarrow \mathbb{R}$ por

$$
f_{x}(t):=\left\{\begin{array}{ll}
1 & \text { se } t \in N_{x} \\
0 & \text { se } t \notin N_{x}
\end{array} .\right.
$$

Seja $F_{x}$ a extensão de $f_{x}$ a $\beta \mathbb{N}$. Em virtude da continuidade de $F_{x}$, o conjunto $F_{x}^{-1}(] \frac{1}{2}, \frac{3}{2}[)$ é aberto. A densidade de $\mathbb{N}$ em $\beta \mathbb{N}$ implica $F_{x}^{-1}(] \frac{1}{2}, 1[)=F_{x}^{-1}(] 1, \frac{3}{2}[)=\emptyset$, portanto $F_{x}^{-1}(1)=F_{x}^{-1}(] \frac{1}{2}, 1[) \cup F_{x}^{-1}(1) \cup F_{x}^{-1}(] 1, \frac{3}{2}[)=F_{x}^{-1}(] \frac{1}{2}, \frac{3}{2}[)$ é aberto.

Decorre que $F_{x}^{-1}(1) \backslash{\overline{N_{x}}}^{\beta \mathbb{N}}$ é aberto. Se existisse $n \in\left(F_{x}^{-1}(1) \backslash \overline{N_{x}}\right) \cap \mathbb{N}$, então $F_{x}(n)=$ $f_{x}(n)=1$ e consequentemente $n \in N_{x} \subset{\overline{N_{x}}}^{\beta \mathbb{N}}$, uma contradição. Então $\left(F_{x}^{-1}(1) \backslash \overline{N_{x}}\right) \cap \mathbb{N}=$ $\emptyset$ e por $\mathbb{N}$ ser denso em $\beta \mathbb{N}$, temos $F_{x}^{-1}(1) \backslash{\overline{N_{x}}}^{\beta \mathbb{N}}=\emptyset$, ou seja, $F_{x}^{-1}(1) \subseteq \overline{N_{x}}{ }^{\beta \mathbb{N}}$. Como 
claramente vale $\overline{N_{x}}{ }^{\beta \mathbb{N}} \subseteq F_{x}^{-1}(1)$, podemos concluir $F_{x}^{-1}(1)={\overline{N_{x}}}^{\beta \mathbb{N}}$. Segue-se que ${\overline{N_{x}}}^{\beta \mathbb{N}}$ é aberto em $\beta \mathbb{N}$.

Se $x \neq y$, fixemos $u \in{\overline{N_{x}}}^{\beta \mathbb{N}} \cap{\overline{N_{y}}}^{\beta \mathbb{N}}$. De acordo com a proposição 1.1.2, existem redes $\left(x_{\gamma}\right)_{\gamma \in \Gamma}$ e $\left(y_{\lambda}\right)_{\lambda \in \Lambda}$ em $N_{x}$ e $N_{y}$ respectivamente, convergindo a $u$. Se $u \notin \mathbb{N}$, da continuidade de $F_{x}$ e por $N_{x} \cap N_{y}$ ser finito, temos

$$
1=\lim _{\gamma} F_{x}\left(x_{\gamma}\right)=F_{x}(u)=\lim _{\beta} F_{x}\left(y_{\beta}\right)=0,
$$

uma contradição. Concluímos que ${\overline{N_{x}}}^{\beta \mathbb{N}} \cap{\overline{N_{y}}}^{\beta \mathbb{N}} \subseteq \mathbb{N}$.

Para cada $x \in \mathbb{R} \backslash \mathbb{Q}$ definimos

$$
W_{x}:=(\beta \mathbb{N} \backslash \mathbb{N}) \cap{\overline{N_{x}}}^{\beta \mathbb{N}} .
$$

De acordo com o que discutimos acima, $\left\{W_{x}: x \in \mathbb{R} \backslash \mathbb{Q}\right\}$ é uma coleção, com a cardinalidade do continuum, de abertos de $\beta \mathbb{N} \backslash \mathbb{N}$ satisfazendo

$$
W_{x} \cap W_{y}=(\beta \mathbb{N} \backslash \mathbb{N}) \cap{\overline{N_{x}}}^{\beta \mathbb{N}} \cap{\overline{N_{y}}}^{\beta \mathbb{N}}=\emptyset,
$$

sempre que $x \neq y$.

Teorema 3.4.11. Se $K$ é um espaço topológico de Hausdorff compacto não satisfazendo a $C C C$ então $\mathcal{C}(K)$ contém um subespaço $Y$, tal que para algum $\Gamma$ não enumerável, $c_{0}(\Gamma) \cong Y$.

Demonstração. Se $K$ não satisfaz a condição $C C C$ então existe uma coleção $\left\{V_{\lambda}: \lambda \in \Gamma\right\}$ de abertos dois a dois disjuntos de $K$, onde $\Gamma$ é um conjunto não enumerável.

Por $K$ ser um espaço topológico de Hausdorff e compacto, $K$ é um espaço topológico normal. Então para cada $\lambda \in \Gamma$, o Lema de Urysohn implica a existência de $f_{\lambda} \in \mathcal{C}(K)$ satisfazendo

$$
f_{\lambda}\left(K \backslash V_{\lambda}\right) \subset\{0\} \text { e }\left\|f_{\lambda}\right\|=1 .
$$

Seja $Y:=\overline{\left[\left\{f_{\lambda}: \lambda \in \Gamma\right\}\right]}$ e para cada $\lambda \in \Gamma$, seja $\|\cdot\|_{\lambda}: \mathcal{C}(K) \rightarrow \mathbb{R}$ definida por

$$
\|\varphi\|_{\lambda}:=\sup _{t \in V_{\lambda}}|\varphi(t)| .
$$

Demonstra-se que se $\varphi \in Y$, então $\|\varphi\|=\sup _{\lambda \in \Gamma}\|\varphi\|_{\lambda}$. Em particular, se $\varphi=b_{1} f_{\lambda_{1}}+$ $\ldots+b_{r} f_{\lambda_{r}}$ então $\|\varphi\|=\max _{1 \leq k \leq r}\left|b_{k}\right|$.

Fixado $\varphi \in Y$ seja $\left(\varphi_{n}\right)_{n \in \mathbb{N}} \subset\left[\left\{f_{\lambda}: \lambda \in \Gamma\right\}\right]$ uma sequência convergindo a $\varphi$. Existe $\left\{\lambda_{k}: k \in \mathbb{N}\right\} \subset \Gamma$ tal que $\varphi_{n} \in\left[\left\{f_{\lambda_{k}}: k \in \mathbb{N}\right\}\right]$ para todo $n \in \mathbb{N}$. 
Se $M:=\sup _{n \in \mathbb{N}}\left\|\varphi_{n}\right\|$, para cada $k \in \mathbb{N}$ existe $a_{\lambda_{k}} \in \mathbb{R}$ satisfazendo

$$
\left\|\varphi-a_{\lambda_{k}} f_{\lambda_{k}}\right\|_{\lambda_{k}}=i n f_{-M \leq x \leq M}\left\|\varphi-x f_{\lambda_{k}}\right\|_{\lambda_{k}}
$$

Definimos a sequência $\left(\phi_{n}\right)_{n \in \mathbb{N}}$ por

$$
\phi_{n}:=\sum_{k=1}^{n} a_{\lambda_{k}} f_{\lambda_{k}} .
$$

Vamos demonstrar que $\phi_{n} \rightarrow \varphi$. Com efeito, fixando-se $\epsilon>0$, por $\varphi_{n} \rightarrow \varphi$, existe $k_{0} \in \mathbb{N}$ tal que $\left\|\varphi-\varphi_{k}\right\|<\epsilon$ sempre que $k \geq k_{0}$. Seja $n_{0} \in \mathbb{N}$, tal que $\varphi_{k_{0}}=b_{1} f_{\lambda_{1}}+\ldots+b_{n_{0}} f_{\lambda_{n_{0}}}$. Temos

$$
\left\|\varphi-\varphi_{k_{0}}\right\|_{\lambda}= \begin{cases}\left\|\varphi-b_{r} f_{\lambda_{r}}\right\|_{\lambda_{r}}<\epsilon & \text { se } \lambda=\lambda_{r}, r \in\left\{1, \ldots, n_{0}\right\}, \\ \|\varphi\|_{\lambda}<\epsilon & \text { se } \lambda \notin\left\{\lambda_{1}, \ldots, \lambda_{n_{0}}\right\} .\end{cases}
$$

Então, se $n>n_{0}$, de acordo com a definição dos $a_{\lambda_{k}}$

$$
\left\|\varphi-\phi_{n}\right\|_{\lambda}= \begin{cases}\left\|\varphi-a_{\lambda_{k}} f_{\lambda_{k}}\right\|_{\lambda_{k}} \leq\left\|\varphi-b_{k} f_{\lambda_{k}}\right\|_{\lambda_{k}}<\epsilon & \text { se } \lambda=\lambda_{r}, r \in\left\{1, \ldots, n_{0}\right\}, \\ \left\|\varphi-a_{\lambda_{k}} f_{\lambda_{k}}\right\|_{\lambda_{k}} \leq\left\|\varphi-0 f_{\lambda_{k}}\right\|_{\lambda_{k}}<\epsilon & \text { se } \lambda=\lambda_{r}, r \in\left\{n_{0}+1, \ldots, n\right\}, \\ \|\varphi\|_{\lambda}<\epsilon & \text { se } \lambda \notin\left\{\lambda_{1}, \ldots, \lambda_{n}\right\} .\end{cases}
$$

Consequentemente, para todo $n \geq n_{0}$

$$
\left\|\varphi-\phi_{n}\right\|=\sup _{\lambda \in \Gamma}\left\|\varphi-\phi_{n}\right\|_{\lambda} \leq \epsilon
$$

Portanto $\phi=\lim _{n \rightarrow \infty} \phi_{n}=\sum_{k=1}^{\infty} a_{\lambda_{k}} f_{\lambda_{k}}$ e verifica-se facilmente que a série converge incondicionalmente. Podemos definir para cada $\lambda \in \Gamma$

$$
a_{\lambda}:=\left\{\begin{array}{ll}
a_{\lambda_{k}} & \text { se } \lambda \in\left\{\lambda_{k}: k \in \mathbb{N}\right\} \\
0 & \text { se } \lambda \notin\left\{\lambda_{k}: k \in \mathbb{N}\right\}
\end{array},\right.
$$

e por abuso de notação, escrever $\varphi=\sum_{\lambda \in \Gamma} a_{\lambda} f_{\lambda}$. Conluímos que cada $\varphi \in Y$ pode ser escrito dessa forma e neste caso temos também, $\|\varphi\|=\sup _{\lambda \in \Gamma}\left|a_{\lambda}\right|$.

Se $\left\{e_{\lambda}: \lambda \in \Gamma\right\}$ é a base canônica de $c_{0}(\Gamma)$, definimos a aplicação $\Psi: Y \rightarrow c_{0}(\Gamma)$, por

$$
\Psi(\varphi)=\Psi\left(\sum_{\lambda \in \Gamma} a_{\lambda} f_{\lambda}\right):=\sum_{\lambda \in \Gamma} a_{\lambda} e_{\lambda}
$$

Sem dificuldade demonstra-se que $\Psi$ é um isomorfismo isométrico. 
Corolário 3.4.12. Seja K um espaço compacto de Hausdorff não satisfazendo a CCC. Para qualquer inteiro positivo $n$, todo polinômio n-homogêneo definido sobre $\mathcal{C}(K)$ e assumindo valores reais, se anula em um subespaço isometricamente isomorfo a $c_{0}(\Gamma)$, onde $\Gamma$ é um conjunto não enumerável.

Demonstração. De acordo com o teorema 3.4 .11 acima, existe um conjunto não enumerável $\Gamma$ e uma isometria linear $T: c_{0}(\Gamma) \rightarrow \mathcal{C}(K)$. Para todo polinômio $P \in \mathcal{C}(K), Q:=P \circ T$ é um polinômio em $\mathcal{P}\left({ }^{n} c_{0}(\Gamma)\right)$.

De acordo com a proposição 3.4.7, $Q$ se anula em um subespaço fechado $Y \subseteq c_{0}(\Gamma)$, isometricamente isomorfo a $c_{0}(\Gamma)$. Consequentemente, $Z:=T(Y)$ é um subespaço de $\mathcal{C}(K)$, isometricamente isomorfo a $c_{0}(\Gamma)$ e tal que $Z \subset P^{-1}(0)$.

Procedendo-se como na demonstração acima e utilizando-se o corolário 3.4.8 demonstrase o seguinte corolário.

Corolário 3.4.13. Seja $\left\{P_{k}: k \in \mathbb{N}\right\}$ uma coleção de polinômios homogêneos em $\mathcal{C}(K)$ onde $K$ é um espaço topológico de Hausdorff não satisfazendo a CCC. Então existe um subespaço $Y \subseteq \bigcap_{k \in \mathbb{N}} P_{k}^{-1}(0)$, tal que $Y \cong c_{0}(\Gamma)$ onde $\Gamma$ é um conjunto não enumerável.

A proposição a seguir pode ser encontrada em [15].

Proposição 3.4.14. $l_{\infty} / c_{0} \cong \mathcal{C}(\beta \mathbb{N} \backslash \mathbb{N})$

Corolário 3.4.15. Seja $\left\{P_{k}: k \in \mathbb{N}\right\}$ uma coleção de polinômios homogêneos definidos em $l_{\infty} / c_{0}$. Então existe um subespaço $Y \subset \bigcap_{k \in \mathbb{N}} P_{k}^{-1}(0)$, tal que $Y \cong c_{0}(\Gamma)$ onde $\Gamma$ é um conjunto com a cardinalidade do continuum.

Demonstração. De acordo com o exemplo 3.4.10, existe uma coleção de abertos dois a dois disjuntos em $\beta \mathbb{N} \backslash \mathbb{N}$ com a cardinalidade do continuum. Procedendo-se como na demonstração da proposição 3.4.11, se obtém um subespaço $Y \subset \mathcal{C}(\beta \mathbb{N} \backslash \mathbb{N})$, tal que $Y \cong c_{0}(\Gamma)$, onde $\Gamma$ é um conjunto com a cardinalidade do continuum. A tese decorre do corolário 3.4.8 e da proposição 3.4.14.

Corolário 3.4.16. Para cada $n \in \mathbb{N}$, se $P \in \mathcal{P}\left({ }^{n} l_{\infty}\right)$ é tal que ker $\partial^{n-1} P$ contém $c_{0}$, então $l_{\infty}$ admite um subespaço fechado $Z$ tal que $c_{0} \subset Z \subset P^{-1}(0)$ e $Z / c_{0} \cong c_{0}(\Gamma)$ onde $\Gamma$ é um conjunto com a cardinalidade do continuum. 
Demonstração. Seja $P \in \mathcal{P}\left({ }^{n} l_{\infty}\right)$ tal que $c_{0} \subset \operatorname{ker} \partial^{n-1} P$. Definimos $\widetilde{P}: l_{\infty} / c_{0} \rightarrow \mathbb{R}$ por

$$
\widetilde{P}\left(x+c_{0}\right):=P(x), x \in l_{\infty} .
$$

Verifica-se que $\widetilde{P}$ define um polinômio homogêneo contínuo sobre $l_{\infty} / c_{0}$. De acordo com o corolário 3.4.15, $l_{\infty} / c_{0}$ admite um subespaço $Y$, tal que $Y \subseteq \widetilde{P}^{-1}(0)$ e $Y \cong c_{0}(\Gamma)$, onde $\Gamma$ tem a cardinalidade do continuum.

Se $\pi: l_{\infty} \rightarrow l_{\infty} / c_{0}$ é a projeção definida por

$$
\pi(x):=x+c_{0}, x \in l_{\infty},
$$

fixamos $Z:=\pi^{-1}(Y)$. Claramente $Z \subset P^{-1}(0)$ e $Z / c_{0} \cong c_{0}(\Gamma)$. 


\section{Capítulo 4}

\section{Lema de Phelps Multilinear}

Nosso principal objetivo neste capítulo é exibir uma versão multilinear para o lema de Phelps, resultado publicado em [3] devido a R. Aron, A. Cardwell, D. García e I. Zalzuendo.

Essencialmente, esse capítulo é baseado nos artigos [3], [5] e [18].

\subsection{Complexificação de Espaços de Banach Reais}

Nesta seção trataremos alguns procedimentos de complexificação de espaços de Banach reais. Se trata de um pequeno estudo do artigo [18], e dará sustentação a alguns resultados que serão estudados na próxima seção.

Para uma abordagem completa sobre o assunto recomendamos [18].

Definição 4.1.1. Um espaço vetorial complexo $\widetilde{X}$ é uma complexificação de um espaço vetorial real $X$ se satisfaz as seguintes condições:

(i) existe uma aplicação $\mathbb{R}$-linear injetora c $: X \rightarrow \widetilde{X}$;

(ii) $[\mathrm{c}(X)]_{\mathbb{C}}=\tilde{X}$.

Exemplo 4.1.2. Se $X$ é um espaço vetorial real, podemos definir sobre $X \times X$ operações de adição e multiplicação por escalar como abaixo:

$$
\begin{aligned}
& (x, y)+(u, v):=(x+u, y+v), \\
& (a+i b)(x, y):=(a x-b y, b x+a y),
\end{aligned}
$$

onde $x, y, u, v \in X$ e $a, b \in \mathbb{R}$. 
Verifica-se sem dificuldade que sob as operações acima, $X \times X$ se torna um espaço vetorial complexo e que a aplicação c : $X \rightarrow X \times X$ definida por

$$
\mathrm{c}(x):=(x, 0)
$$

satisfaz as condições $(i)$ e $(i i)$ na definição 4.1.1. Decorre portanto que $X \times X$ é uma complexificação de $X$.

Observemos que a condição $(i)$ na definição 4.1.1 implica $X$ isomorfo a um espaço vetorial real contido em $\tilde{X}$. Por simplicidade é conveniente identificarmos $X \operatorname{com~c}(X)$ de modo a omitir referências à aplicação c. Dessa forma uma complexificação de um espaço vetorial real $X$ será um espaço vetorial complexo $\tilde{X}$ contendo o espaço vetorial real $X$ e tal que $[X]_{\mathbb{C}}=\tilde{X}$ e esta é a identificação que utilizaremos a partir de agora.

Sob esta nova identificação, na complexificação do exemplo 4.1.2, podemos escrever $u=$ $x+i y$ para cada elemento $u=(x, y)=\mathrm{c}(x)+i \mathrm{c}(y)$. Mais geralmente, um elemento arbitrário de uma complexificação $\widetilde{X}$ pode ser escrito como $u=x+i y$, com $x, y \in X$.

Devemos observar que na complexificação do exemplo 4.1.2, se $x+i y=0$, com $x, y \in$ $X$, então $x=y=0$. Em uma complexificação com essa propriedade podemos definir, inequivocamente, para cada $u=x+i y, \operatorname{com} x, y \in X, \Re(u):=x$ e $\Im(u):=y$ que serão respectivamente a parte real e a parte imaginária de $u$. Podemos também definir o conjugado de $u$, por $\bar{u}:=x-i y$.

A partir de agora, $\widetilde{X}$ denotará uma complexificação de um espaço de Banach real $X$ tal que se $x+i y=0$, com $x, y \in X$, então $x=y=0$.

Estudaremos formas de se definir normas sobre complexificações de espaços de Banach reais, satisfazendo certas condições desejáveis.

Definição 4.1.3. Seja $X$ um espaço de Banach real. Dizemos que uma norma $\|\cdot\|_{\tilde{X}}$ sobre $\widetilde{X}$ é razoável se para quaiquer $x, y \in X$

(iii) $\|x\|_{\tilde{X}}=\|x\|_{X}$

(iv) $\|x+i y\|_{\widetilde{X}}=\|x-i y\|_{\widetilde{X}}$.

O espaço $\widetilde{X}$ munido de uma norma $\|\cdot\|_{\widetilde{X}}$ satisfazendo as condições $(i i i)$ e $(i v)$ será chamado de complexificação razoável de $X$. Uma norma $\|\cdot\|_{\tilde{X}}$ nessas condições, será chamada norma razoável sobre $\tilde{X}$.

As condições (iii) e (iv) na definição 4.1 .3 são baseadas nas seguintes propriedades básicas dos números complexos. Para todo $x \in \mathbb{C}$ temos $|x|=|x+i 0|$ e se $z$ é um complexo seu 
conjugado $\bar{z}$ satisfaz $|z|=|\bar{z}|$. Claramente se $X$ for um espaço de Banach real, sob uma norma razoável $\|\cdot\|_{\tilde{X}}$, o espaço $\widetilde{X}$ é um espaço de Banach complexo.

O próximo resultado nos permitirá sempre definir uma norma razoável sobre a complexificação de um espaço de Banach real. Ficará estabelecido também que quaisquer normas razoáveis são equivalentes.

Proposição 4.1.4. Seja $\widetilde{X}$ uma complexificação de um espaço de Banach real X. Então a aplicação $\|\cdot\|_{T}: \widetilde{X} \rightarrow \mathbb{R}$ definida por

$$
\|x+i y\|_{T}:=\sup _{0 \leq t \leq 2 \pi}\|x \cos t-y \sin t\|,
$$

é uma norma razoável sobre $\widetilde{X}$. Além disso se $\|\cdot\|_{\tilde{X}}$ for alguma outra norma razoável sobre $\widetilde{X}$ então

$$
\|x+i y\|_{T} \leq\|x+i y\|_{\tilde{X}} \leq 2\|x+i y\|_{T} .
$$

Demonstração. A verificação de que $\|\cdot\|_{T}$ define uma norma sobre $\widetilde{X}$ é direta. Para verificarmos a validade dos items (iii) e (iv) na definição 4.1.3, fixemos $x, y \in X$. Claramente

$$
\|x\|_{T}=\sup _{0 \leq t \leq 2 \pi}\|x \cos t-0 \sin t\|=\|x\|,
$$

o que verifica (iii). Temos também

$$
\begin{aligned}
\|x+i y\|_{T} & =\sup _{0 \leq t \leq 2 \pi}\|x \cos t-y \sin t\|=\sup _{0 \leq t \leq 2 \pi}\|x \cos (\pi-t)+y \sin (\pi-t)\| \\
& =\sup _{\pi \leq r \leq 3 \pi}\|x \cos r+y \sin r\|=\sup _{0 \leq r \leq 2 \pi}\|x \cos r-(-y) \sin r\|=\|x-i y\|_{T},
\end{aligned}
$$

o que verifica $(i v)$. Fica estabelecido portanto que $\|\cdot\|_{T}$ é uma norma razoável sobre $\widetilde{X}$. Verificaremos agora que qualquer outra norma razoável é equivalente a esta.

Com efeito, se $\|\cdot\|_{\tilde{X}}$ for uma outra norma razoável, de acordo com a propriedade (iii), para quaisquer $x, y \in X$, teremos

$$
2\|x\|=2\|x\|_{\tilde{X}}=\|x+i y+x-i y\|_{\tilde{X}} \leq\|x+i y\|_{\tilde{X}}+\|x-i y\|_{\tilde{X}} .
$$

Então, em virtude da condição $(i v)$,

$$
\|x\| \leq\|x+i y\|_{\tilde{X}}, x, y \in X .
$$


Consequentemente, para todo $0 \leq t \leq 2 \pi$,

$$
\begin{aligned}
\|x+i y\|_{\tilde{X}} & =\left\|e^{i t}(x+i y)\right\|_{\tilde{X}} \\
& =\|(x \cos t-y \sin t)+i(x \sin t+y \cos t)\|_{\tilde{X}} \\
& \geq\|x \cos t-y \sin t\| .
\end{aligned}
$$

Portanto $\|x+i y\|_{\tilde{X}} \geq\|x+i y\|_{T}$. Por outro lado, a desigualdade triangular e a condição (iii) implicam

$$
\begin{aligned}
\|x+i y\|_{\tilde{X}} & =\left\|e^{i t}(x+i y)\right\|_{\tilde{X}}=\|(x \cos t-y \sin t)+i(x \sin t+y \cos t)\|_{\tilde{X}} \\
& \leq\|x \cos t-y \sin t\|_{\tilde{X}}+\|x \sin t+y \cos t\|_{\tilde{X}} \\
& =\|x \cos t-y \sin t\|+\|x \sin t+y \cos t\| \\
& \leq 2\|x+i y\|_{T},
\end{aligned}
$$

como queríamos.

Em [18] é exibido um exemplo de norma razoável onde ocorre $\|x+i y\|_{\tilde{X}}=2\|x+i y\|_{T}$.

A norma $\|\cdot\|_{T}$ foi utilizada primeiramente por A. E. Taylor em [23], reaparecendo posteriormente em diversos trabalhos. Em [18], os autores se referem a $\left(\widetilde{X},\|\cdot\|_{T}\right)$ como complexificação de Taylor de $X$.

Utilizando-se o teorema de Hahn-Banach, podemos obter uma descrição alternativa de $\|x+i y\|_{T}:$

$$
\begin{aligned}
\|x+i y\|_{T} & =\sup _{0 \leq t \leq 2 \pi}\|x \cos t-y \sin t\| \\
& =\sup _{0 \leq t \leq 2 \pi} \sup _{\varphi \in B_{X^{*}}}|\varphi(x) \cos t-\varphi(y) \sin t| \\
& =\sup _{\varphi \in B_{X^{*}}} \sqrt{\varphi(x)^{2}+\varphi(y)^{2}} .
\end{aligned}
$$

Uma característica importante da complexificação de Taylor é ser um procedimento de complexificação que independe de quaisquer características específicas de um espaço de Banach real $X$, ou seja, a definição independe do espaço do espaço de Banach real a ser complexificado. Veremos na proposição 4.1.5 que esse procedimento nos permite estender aplicações $\mathbb{R}$-lineares contínuas entre espaços de Banach reais $X, Y$ para aplicações $\mathbb{C}$-lineares entre suas respectivas complexificações $\widetilde{X}, \widetilde{Y}$ sem alterarmos a norma.

Do ponto de vista algébrico, se $X$ e $Y$ são espaços vetoriais reais, dada uma aplicação 
$\mathbb{R}$-linear $A: X \rightarrow Y$, verifica-se facilmente que a aplicação $\widetilde{A}: \widetilde{X} \rightarrow \widetilde{Y}$ definida de maneira natural

$$
\widetilde{A}(x+i y):=A(x)+i A(y),
$$

é a única extensão $\mathbb{C}$-linear possível entre $\widetilde{X}$ e $\widetilde{Y}$.

Proposição 4.1.5. Sejam $X$ e $Y$ espaços de Banach reais. Se $A \in \mathcal{L}(X, Y)$ então a aplicação $\widetilde{A}$ definida como em (4.1) satisfaz

$$
\widetilde{A} \in \mathcal{L}\left(\left(\widetilde{X},\|\cdot\|_{T}\right),\left(\widetilde{Y},\|\cdot\|_{T}\right)\right) e\|\widetilde{A}\|_{T}=\|A\|
$$

Demonstração. Para cada $x, y \in X$ temos

$$
\begin{aligned}
\|\widetilde{A}(x+i y)\|_{T} & =\|A(x)+i A(y)\|_{T}=\sup _{0 \leq t \leq 2 \pi}\|A(x) \cos t-A(y) \sin t\| \\
& =\sup _{0 \leq t \leq 2 \pi}\|A(x \cos t-y \sin t)\| \leq\|A\| \sup _{0 \leq t \leq 2 \pi}\|x \cos t-y \sin t\| \\
& =\|A\|\|x+i y\|_{T} .
\end{aligned}
$$

Portanto $\widetilde{A} \in \mathcal{L}\left(\left(\widetilde{X},\|\cdot\|_{T}\right),\left(\widetilde{Y},\|\cdot\|_{T}\right)\right)$ e $\|\widetilde{A}\|_{T} \leq\|A\|$. Como $\widetilde{A}$ é extensão de $A$, temos $\|A\| \leq\|\widetilde{A}\|_{T}$.

Existem diversos outros procedimentos de complexificação com propriedades similares ao procedimento de complexificação de Taylor.

Definição 4.1.6. Um procedimento de complexificação natural é uma maneira de se definir uma norma razoável $\|\cdot\|_{\gamma}$, sobre uma complexificação de qualquer espaço de Banach real, satisfazendo a seguinte propriedade:

(v) se $X, Y$ forem espaços de Banach reais, para toda aplicação linear $A \in \mathcal{L}(X, Y)$, a extensão linear complexa, $\widetilde{A}:\left(\widetilde{X},\|\cdot\|_{\gamma}\right) \rightarrow\left(\widetilde{Y},\|\cdot\|_{\gamma}\right)$, satisfaz $\|\widetilde{A}\|_{\gamma}=\|A\|$.

Nessas condições, o espaço $\widetilde{X}$ munido da norma $\|\cdot\|_{\gamma}$ será chamado de complexificação natural de $X$. A norma $\|\cdot\|_{\gamma}$ será chamada de norma natural complexa sobre $\widetilde{X}$.

O exemplo a seguir fornece uma coleção de procedimentos de complexificação natural.

Exemplo 4.1.7. Seja $\widetilde{X}$ uma complexificação de um espaço de Banach real $X$. Para cada $x, y \in X$ definimos

$$
\begin{aligned}
n_{p}(x+i y) & :=\left(\|x\|^{p}+\|y\|^{p}\right)^{\frac{1}{p}} \text { se } 1 \leq p<\infty, \\
n_{\infty}(x+i y) & :=\max \{\|x\|,\|y\|\}
\end{aligned}
$$


Verifica-se facilmente que para cada $1 \leq p \leq \infty$, em geral não vale a relação

$$
n_{p}(\lambda(x+i y))=|\lambda| n_{p}(x+i y) \text { para todo } \lambda \in \mathbb{C}
$$

e portanto $n_{p}$ não define norma sobre $\tilde{X}$. No entanto, para cada $1 \leq p \leq \infty$ podemos, a partir de $n_{p}$, obter uma norma $N_{p}$ sobre $\widetilde{X}$, definindo-se

$$
N_{p}(x+i y):=\sup _{0 \leq t \leq 2 \pi} n_{p}\left(e^{t i}(x+i y)\right)
$$

De fato, para cada $\lambda \in \mathbb{C}$ existe $0 \leq r \leq 2 \pi$ tal que $\lambda=|\lambda| e^{r i}$. Então

$$
\begin{aligned}
N_{p}(\lambda(x+i y)) & =\sup _{0 \leq t \leq 2 \pi} n_{p}\left(\lambda e^{i t}(x+i y)\right)=\sup _{0 \leq t \leq 2 \pi} n_{p}\left(|\lambda| e^{(r+t) i}(x+i y)\right) \\
& =|\lambda| \sup _{0 \leq s \leq 2 \pi} n_{p}\left(e^{s i}(x+i y)\right)=|\lambda| N_{p}(x+i y) .
\end{aligned}
$$

As demais propriedades de norma podem ser facilmente verificadas, portanto serão omitidas.

Fixando-se $1 \leq p \leq \infty, x, y \in X$ temos

$$
\begin{aligned}
N_{p}(x-i y) & =\sup _{0 \leq t \leq 2 \pi} n_{p}\left(e^{t i}(x-i y)\right) \\
& =\sup _{0 \leq t \leq 2 \pi} n_{p}\left(e^{-t i}(x+i y)\right)=N_{p}(x+i y) .
\end{aligned}
$$

Isso demonstra que para cada $1 \leq p \leq \infty$, a norma $N_{p}$ satisfaz a propriedade $(i v)$ da definição 4.1.3. Diretamente da definição de $N_{P}$, para cada $x \in X$,

$$
\begin{aligned}
& N_{p}(x)=\|x\| \sup _{0 \leq t \leq 2 \pi}\left(|\cos t|^{p}+|\sin t|^{p}\right)^{\frac{1}{p}} \quad \text { se } 1 \leq p<\infty \\
& N_{\infty}(x)=\|x\| .
\end{aligned}
$$

Utilizando-se resultados elementares de Cálculo obtemos

$$
\sup _{0 \leq t \leq 2 \pi}\left(|\cos t|^{p}+|\sin t|^{p}\right)^{\frac{1}{p}}=\left\{\begin{array}{ll}
1 & \text { se } 2 \leq p<\infty \\
2^{1 / p-1 / 2} & \text { se } 1 \leq p<2
\end{array} .\right.
$$

Concluímos que se $2 \leq p \leq \infty$ então $N_{p}$ também satisfaz a propriedade (iii) da definição 4.1.3, e portanto define uma norma razoável sobre $\widetilde{X}$. Se $1 \leq p \leq 2$ nos resta um fator constante $2^{(1 / p-1 / 2)}$. Realizando-se então um pequeno ajuste, podemos para cada $1 \leq p \leq \infty$ 
definir uma norma razoável sobre $\tilde{X}$

$$
\begin{array}{rlrl}
\|x+i y\|_{(p)}: & =N_{p}(x+i y) & \text { se } 2 \leq p<\infty, \\
\|x+i y\|_{(p)}:=2^{(1 / 2-1 / p)} N_{p}(x+i y) & \text { se } 1 \leq p<2 .
\end{array}
$$

Obtemos, portanto, uma maneira de se definir, para cada $1 \leq p \leq \infty$, uma norma razoável $\|\cdot\|_{(p)}$ sobre uma complexificação de qualquer espaço de Banach real. Diretamente da definição de $\|\cdot\|_{(p)}$, verifica-se que este procedimento satisfaz a condição $(v)$ da definição 4.1.6, se tratando portanto de um procedimento de complexificação natural.

\subsection{Extensões Complexas de Multilineares e Polinômios Reais}

A partir de agora nosso foco será o estudo de extensões complexas de aplicações multilineares e polinômios reais. O principal resultado exibido nesta seção nos permitirá estender algumas proposições para polinômios reais a partir de proposições previamente obtidas para polinômios complexos.

Sejam $X$ e $Y$ espaços de Banach sobre $\mathbb{R}$ e $\widetilde{X}$ e $\widetilde{Y}$ complexificações de $X$ e $Y$ respectivamente. Os matemáticos J. Bochnak e J. Siciak observaram (ver Teorema 3 em [7]), que toda aplicação $A \in \mathcal{L}\left({ }^{n} X, Y\right)$ admite uma única extensão complexa $\widetilde{A} \in \mathcal{L}\left({ }^{n} \widetilde{X}, \widetilde{Y}\right)$ definida por por

$$
\widetilde{A}\left(x_{10}+i x_{11}, \ldots, x_{n 0}+i x_{n 1}\right)=\sum_{\epsilon_{i}=0,1} i^{\sum_{k=1}^{n} \epsilon_{k}} A\left(x_{1 \epsilon_{1}}, \ldots, x_{n \epsilon_{n}}\right),
$$

onde $x_{k 0}, x_{k 1} \in X$ e a soma percorre todas as $2^{n}$ sequências $\left(\epsilon_{1}, \ldots, \epsilon_{n}\right)$, com $\epsilon_{k} \in\{0,1\}$, $1 \leq k \leq n$. A norma de $\widetilde{A}$, depende das normas utilizadas em $\widetilde{X}$ e $\widetilde{Y}$ mas a continuidade é assegurada.

No contexto dos polinômios (ver [23](p. 313)), qualquer $P \in \mathcal{P}_{a}\left({ }^{n} X ; Y\right)$ admite uma única extensão complexa $\widetilde{P} \in \mathcal{P}_{a}\left({ }^{n} \widetilde{X} ; \widetilde{Y}\right)$ definida por

$\widetilde{P}(x+i y)=\sum_{k=0}^{\left\lfloor\frac{n}{2}\right\rfloor}(-1)^{k}\left(\begin{array}{c}n \\ 2 k\end{array}\right) \widehat{P} x^{n-2 k} y^{2 k}+i \sum_{k=0}^{\left\lfloor\frac{n-1}{2}\right\rfloor}(-1)^{k}\left(\begin{array}{c}n \\ 2 k+1\end{array}\right) \widehat{P} x^{n-(2 k+1)} y^{2 k+1}, x, y \in X$,

onde para cada $t \in \mathbb{R},\lfloor t\rfloor:=\max \{n \in \mathbb{N}: n \leq t\}$.

Em geral, qualquer polinômio contínuo $P=\sum_{k=0}^{n} P_{k} \operatorname{com} P_{k} \in \mathcal{L}\left({ }^{k} X ; Y\right), 0 \leq k \leq n$, admite uma única extensão complexa $\widetilde{P}=\sum_{k=0}^{n} \widetilde{P}_{k}$.

Em contraste com a proposição 4.1.5, no caso polinomial raramente ocorre $\|P\|=\|\widetilde{P}\|$. 
Para mais detalhes ver [18].

O resultado principal desta seção é um teorema devido a G. A. Muñoz, Y. Sarantopoulos e A. Tonge, publicado em [18]. Para demonstrá-lo, precisamos de um pequeno lema.

Lema 4.2.1. Para quaisquer $n, k \in \mathbb{N}$

$$
\frac{1}{2 n} \sum_{r=0}^{2 n-1}(-1)^{r} e^{i k r \frac{\pi}{n}}=\left\{\begin{array}{ll}
1 & \text { se } k \equiv n(\bmod 2 n) \\
0 & \text { caso contrário }
\end{array} .\right.
$$

Demonstração. Se $k \equiv n(\bmod 2 n)$ então $e^{i k \frac{\pi}{n}}=-1, \operatorname{logo}$

$$
\frac{1}{2 n} \sum_{r=0}^{2 n-1}(-1)^{r} e^{i k r \frac{\pi}{n}}=\frac{1}{2 n} \sum_{r=0}^{2 n-1}(-1)^{r}(-1)^{r}=1
$$

Caso contrário temos $e^{i k \frac{\pi}{n}} \neq-1$ e utilizando a conhecida identidade polinomial

$$
\left(x^{2 n-1}+x^{2 n-2}+\ldots+x^{2}+x+1\right)(x-1)=x^{2 n}-1,
$$

obtemos

$$
\left(\sum_{r=0}^{2 n-1}(-1)^{r} e^{i k r \frac{\pi}{n}}\right)\left(-e^{i k \frac{\pi}{n}}-1\right)=\left(-e^{i k \frac{\pi}{n}}\right)^{2 n}-1=0,
$$

concluindo a demonstração.

Teorema 4.2.2. Sejam $X$ um espaço de Banach real e $\left(\widetilde{X},\|\cdot\|_{\gamma}\right)$ uma complexificação natural de $X$. Se $P \in \mathcal{P}\left({ }^{n} X\right)$ e $\widetilde{P} \in \mathcal{P}\left({ }^{n}\left(\widetilde{X},\|.\|_{\gamma}\right)\right)$ é a extensão complexa de $P$ sobre $\widetilde{X}$ entẫo

$$
\|\widetilde{P}\|_{\gamma} \leq 2^{n-1}\|P\|
$$

Demonstração. Fixado $u=x+i y \in \widetilde{X}$ com $\|u\|_{\gamma} \leq 1$, seja $f: \mathbb{R} \rightarrow \mathbb{R}$ definida por

$$
f(t):=\widetilde{P}\left(\frac{u e^{i t}+\bar{u} e^{-i t}}{2}\right) .
$$

Claramente $f(t)=P(x \cos t-y \sin t)$ e em virtude de $\|\cdot\|_{\gamma}$ ser uma norma natural, decorre, pela proposição 4.1.4,

$$
\begin{aligned}
|f(t)| & =|P(x \cos t-y \sin t)| \leq\|P\|\|x \cos t-y \sin t\| \\
& \leq\|P\|\|x+i y\|_{T} \leq\|P\|\|x+i y\|_{\gamma}=\|P\|,
\end{aligned}
$$


ou seja,

$$
|f(t)| \leq\|P\| .
$$

Consideremos agora $A \in \mathcal{L}^{s}\left({ }^{n} \widetilde{X}\right)$ tal que $\widetilde{P}=\check{A}$. A Fórmula do Binômio 2.2.6 implica

$$
f(t)=\widetilde{P}\left(\frac{u e^{i t}+\bar{u} e^{-i t}}{2}\right)=\sum_{k=0}^{n} \frac{e^{i(n-2 k) t}}{2^{n}}\left(\begin{array}{l}
n \\
k
\end{array}\right) A u^{n-k} \bar{u}^{k} .
$$

Podemos escrever

$$
f(t)=\sum_{k=-n}^{n} a_{k} e^{i k t}
$$

onde $a_{k} \in \mathbb{C}$ para todo $-n \leq k \leq n$. Em particular,

$$
a_{n}=\frac{e^{i n t}}{2^{n}} \widetilde{P}(u), a_{-n}=\frac{e^{-i n t}}{2^{n}} \widetilde{P}(\bar{u}) .
$$

Utilizando (4.3), (4.4) e o lema 4.2.1, decorre

$$
\begin{aligned}
\frac{1}{2 n} \sum_{k=0}^{2 n-1}(-1)^{k} f\left(t+k \frac{\pi}{n}\right) & =\frac{1}{2 n} \sum_{k=0}^{2 n-1}(-1)^{k} \sum_{r=-n}^{n} a_{r} e^{i r\left(t+k \frac{\pi}{n}\right)} \\
& =\sum_{r=-n}^{n} a_{r} e^{i r t}\left\{\frac{1}{2 n} \sum_{k=0}^{2 n-1}(-1)^{k} e^{i r k \frac{\pi}{n}}\right\} \\
& =a_{n} e^{i n t}+a_{-n} e^{-i n t} \\
& =\frac{e^{i n t}}{2^{n}} \widetilde{P}(u)+\frac{e^{-i n t}}{2^{n}} \widetilde{P}(\bar{u}),
\end{aligned}
$$

e pela relação $(4.2)$

$$
\sup _{0 \leq t \leq 2 \pi}\left|e^{i n t} \widetilde{P}(u)+e^{-i n t} \widetilde{P}(\bar{u})\right| \leq 2^{n}\|P\| .
$$

Em virtude de $\widetilde{P}(\bar{u})=\widetilde{P}(u)$ e pelo fato de existir $0 \leq \theta \leq 2 \pi$ tal que $|\widetilde{P}(u)|=\widetilde{P}(u) e^{i \theta}$, podemos escrever

$$
\begin{aligned}
\sup _{0 \leq t \leq 2 \pi}\left|e^{i n t} \widetilde{P}(u)+e^{-i n t} \widetilde{P}(\bar{u})\right| & =\sup _{0 \leq t \leq 2 \pi}\left|e^{i n t} \widetilde{P}(u)+\overline{e^{i n t} \widetilde{P}(u)}\right| \\
& =2 \sup _{0 \leq t \leq 2 \pi}\left|\Re\left(\widetilde{P}(u) e^{i n t}\right)\right| \\
& =2|\widetilde{P}(u)| \sup _{0 \leq t \leq 2 \pi}\left|e^{i(n-\theta) t}\right|=2|\widetilde{P}(u)|,
\end{aligned}
$$


consequentemente

$$
|\widetilde{P}(u)| \leq 2^{n-1}\|P\| .
$$

Como $u$ é arbitrário e $\|u\| \leq 1$, concluímos

$$
\|\widetilde{P}\|_{\gamma} \leq 2^{n-1}\|P\|
$$

Em [18], os autores estabeleceram que esta estimativa é a melhor possível.

\subsection{Produto de Polinômios em Espaços de Banach}

Seja $X$ um espaço de Banach sobre um corpo $\mathbb{K}$. Se $P_{1}, \ldots, P_{n}: X \rightarrow \mathbb{K}$ são polinômios contínuos, o produto $P_{1} \ldots P_{n}$ é um polinômio contínuo e claramente

$$
\left\|\prod_{k=1}^{n} P_{k}\right\| \leq \prod_{k=1}^{n}\left\|P_{k}\right\|
$$

Nesta seção discutiremos um método para obter constantes $M$ dependendo apenas dos graus de $P_{1}, \ldots, P_{n}$, satisfazendo

$$
\prod_{k=1}^{n}\left\|P_{k}\right\| \leq M\left\|\prod_{k=1}^{n} P_{k}\right\| .
$$

Os resultados desta seção foram publicados em [5], são devidos a C. Benítez, Y. Sarantoupoulos e A. Tonge.

No caso em que $\mathbb{K}$ é o corpo dos números complexos o método fornecerá a melhor constante possível, entretanto, quando aplicado ao corpo dos números reais uma constante pode ser obtida mas não a melhor em geral.

O lema a seguir pode ser verificado desenvolvendo-se os polinômios em termos de suas respectivas aplicações multilineares simétricas associadas.

Lema 4.3.1. Para cada $1 \leq k \leq n$, seja $P_{k} \in \mathcal{P}\left({ }^{m_{k}} X\right)$, onde $X$ é um espaço de Banach complexo. Fixado $1 \leq l \leq n$ sejam $m=m_{1}+\ldots+m_{n}$ e $s=m_{1}+. .+m_{l}$. Para quaisquer $x, y \in X$ temos,

$$
\prod_{k=1}^{l} P_{k}(x) \prod_{j=l+1}^{n} \overline{P_{j}(y)}=\frac{1}{2 \pi} \int_{0}^{2 \pi} e^{i(m-s) t} \prod_{k=1}^{l} P_{k}\left(x+e^{i t} y\right) \prod_{j=l+1}^{n} \overline{P_{j}\left(x+e^{i t} y\right)} d t,
$$


onde a barra denota a conjugação complexa.

O lema a seguir será essencial na demonstração do principal resultado desta seção, o teorema 4.3.5.

Lema 4.3.2. Para cada $1 \leq k \leq n$, seja $P_{k} \in \mathcal{P}\left({ }^{m_{k}} X\right)$, onde $X$ é um espaço de Banach complexo. Fixado $1 \leq l \leq n$ sejam $m=m_{1}+\ldots+m_{n}$ e $s=m_{1}+. .+m_{l}$. Se $1 \leq p<\infty e$ $x, y$ são vetores unitários em $X$ satisfazendo,

$$
\left\|z_{1} x+z_{2} y\right\| \leq\left(\left|z_{1}\right|^{p}+\left|z_{2}\right|^{p}\right)^{\frac{1}{p}}, z_{1}, z_{2} \in \mathbb{C},
$$

então

$$
\prod_{j=1}^{l} P_{j}(x) \prod_{i=l+1}^{n} \overline{P_{i}(y)} \leq \frac{m^{\frac{m}{p}}}{s^{\frac{s}{p}}(m-s)^{\frac{m-s}{p}}}\left\|P_{1} \ldots P_{n}\right\| .
$$

Demonstração. Em virtude do lema 4.3.1, para todo $z_{1}, z_{2} \in \mathbb{C}$, temos

$$
\begin{aligned}
\prod_{k=1}^{l}\left|P_{k}\left(z_{1} x\right)\right| \prod_{j=l+1}^{n}\left|P_{j}\left(z_{2} y\right)\right| & =\left|\frac{1}{2 \pi} \int_{0}^{2 \pi} e^{i(m-s) t} \prod_{k=1}^{l} P_{k}\left(z_{1} x+e^{i t} z_{2} y\right) \prod_{j=l+1}^{n} \overline{P_{j}\left(z_{1} x+e^{i t} z_{2} y\right)} d t\right| \\
& \leq \frac{1}{2 \pi} \int_{0}^{2 \pi} \prod_{k=1}^{n}\left|P_{k}\left(z_{1} x+e^{i t} z_{2} y\right)\right| d t \\
& \leq\left\|P_{1} \ldots P_{n}\right\| \frac{1}{2 \pi} \int_{0}^{2 \pi}\left\|z_{1} x+z_{2} e^{i t} y\right\|^{m} d t \\
& \leq\left\|P_{1} \ldots P_{n}\right\|\left(\left|z_{1}\right|^{p}+\left|z_{2}\right|^{p}\right)^{\frac{m}{p}} .
\end{aligned}
$$

Consequentemente

$$
\prod_{k=1}^{l}\left|P_{k}(x)\right| \prod_{j=l+1}^{n}\left|P_{j}(y)\right| \leq \frac{\left(\left|z_{1}\right|^{p}+\left|z_{2}\right|^{p}\right)^{\frac{m}{p}}}{\left|z_{1}\right|^{s}\left|z_{2}\right|^{m-s}}\left\|P_{1} \ldots P_{n}\right\| .
$$

A tese é obtida fixando-se $z_{1}=s^{\frac{1}{p}}$ e $z_{2}=(m-s)^{\frac{1}{p}}$.

O exemplo a seguir nos mostra que a constante do lema anterior não pode ser melhorada em geral.

Exemplo 4.3.3. Fixado $1 \leq p<\infty$, para cada $1 \leq i \leq n$ seja $P_{i} \in \mathcal{P}\left({ }^{m_{i}} l_{p}\right)$ definido por

$$
P_{i}\left(\left(x_{k}\right)_{k \in \mathbb{N}}\right)=x_{\left(m_{1}+\ldots+m_{i-1}+1\right)} \ldots x_{\left(m_{1}+\ldots+m_{i}\right)},
$$


onde $m_{0}:=0$.

Fixado $1 \leq l \leq n, \operatorname{sejam} m=m_{1}+\ldots+m_{n}$ e $s=m_{1}+\ldots+m_{l}$. Se $\left\{e_{k}: k \in \mathbb{N}\right\}$ é a base canônica de $l_{p}$, fixamos $x:=s^{\frac{-1}{p}}\left(e_{1}+\ldots+e_{s}\right)$ e $y:=(m-s)^{\frac{-1}{p}}\left(e_{s+1}+\ldots+e_{m}\right)$.

Demonstra-se facilmente que para cada $z_{1}, z_{2} \in \mathbb{C}$,

$$
\left\|z_{1} x+z_{2} y\right\| \leq\left(\left|z_{1}\right|^{p}+\left|z_{2}\right|^{p}\right)^{\frac{1}{p}} .
$$

Se $1 \leq i \leq l$,

$$
\begin{aligned}
& P_{1}(x)=x_{1} x_{2} \ldots x_{m_{1}}=s^{-\frac{m_{1}}{p}} \\
& P_{2}(x)=x_{\left(m_{1}+1\right)} \ldots x_{\left(m_{1}+m_{2}\right)}=s^{-\frac{m_{2}}{p}} \\
& \quad \vdots \\
& P_{l}(x)=x_{\left(m_{1}+\ldots+m_{l-1}+1\right)} \ldots x_{\left(m_{1}+\ldots+m_{l}\right)}=s^{-\frac{m_{l}}{p}} .
\end{aligned}
$$

Se $l+1 \leq k \leq n$,

$$
\begin{aligned}
& P_{l+1}(y)=y_{\left(m_{1}+\ldots+m_{l}+1\right)} \ldots y_{\left(m_{1}+\ldots+m_{l+1}\right)}=(m-s)^{-\frac{m_{l+1}}{p}} ; \\
& P_{l+2}(y)=y_{\left(m_{1}+\ldots+m_{l+1}+1\right) \ldots y_{\left(m_{1}+\ldots+m_{l+2}\right)}=(m-s)^{-\frac{m_{l+2}}{p}}} ; \\
& \quad \vdots \\
& P_{n}(y)=y_{\left(m_{1}+\ldots+m_{n-1}\right)} \ldots y_{\left(m_{1}+\ldots+m_{n}\right)}=(m-s)^{-\frac{m_{n}}{p}} .
\end{aligned}
$$

Decorre

$$
\begin{aligned}
\prod_{k=1}^{l}\left|P_{i}(x)\right| \prod_{k=l+1}^{n}\left|P_{k}(y)\right| & =s^{\frac{-\left(m_{1}+\ldots+m_{l}\right)}{p}}(m-s)^{\frac{-\left(m_{l+1}+\ldots+m_{n}\right)}{p}} \\
& =s^{\frac{-s}{p}}(m-s)^{\frac{-(m-s)}{p}}
\end{aligned}
$$

Para cada $u=\left(u_{n}\right)_{n \in \mathbb{N}} \in B_{l_{p}}$, em virtude da desigualdade entre as médias aritmética e geométrica,

$$
\begin{aligned}
\left|P_{1}(u) \ldots P_{n}(u)\right| & =\left|u_{1} \ldots u_{m}\right|=\left(\left|u_{1}\right|^{p} \ldots\left|u_{m}\right|^{p}\right)^{\frac{1}{p}} \\
& \leq\left(\frac{\left|u_{1}\right|^{p}+\ldots+\left|u_{m}\right|^{p}}{m}\right)^{\frac{m}{p}} \leq \frac{1}{m^{\frac{m}{p}}}=m^{-\frac{m}{p}} .
\end{aligned}
$$

Consequentemente

$$
\left\|P_{1} \ldots P_{n}\right\| \leq \frac{1}{m^{\frac{m}{p}}}
$$


Por outro lado, fixando-se $u=\frac{1}{m^{\frac{1}{p}}}\left(e_{1}+\ldots+e_{m}\right)$, claramente $\|u\|=1 \mathrm{e}$

$$
\left|P_{1}(u) \ldots P_{n}(u)\right|=\frac{1}{m^{\frac{m}{p}}} .
$$

Então

$$
\left\|P_{1} \ldots P_{n}\right\|=\frac{1}{m^{\frac{m}{p}}}
$$

e portanto,

$$
\prod_{k=1}^{l}\left|P_{i}(x)\right| \prod_{k=l+1}^{n}\left|P_{k}(y)\right|=\frac{m^{\frac{m}{p}}}{s^{\frac{s}{p}}(m-s)^{\frac{(m-s)}{p}}}\left\|P_{1} \ldots P_{n}\right\| .
$$

Com o objetivo de simplificar a demonstração do teorema 4.3.5 mais a diante, estudaremos uma forma de, a partir de um polinômio $P$ definido em algum espaço de Banach complexo, se obter um polinômio $P^{c}$ homogêneo de mesmo grau definido em algum outro espaço de Banach complexo e satisfazendo

$$
\|P\|=\left\|P^{c}\right\|
$$

Dado $X$, um espaço vetorial sobre $\mathbb{C}$, verifica-se que o conjunto $X \times \mathbb{C}$, com as operações de adição de vetores e multiplicação de vetor por escalar, definidas de maneira usual, é um espaço vetorial sobre $\mathbb{C}$. Este espaço será denotado por $X^{c}$ e um elemento arbitrário deste espaço será denotado por $(x, z)$ onde $x \in X$ e $z \in \mathbb{C}$.

Também, verifica-se que se $X$ é um espaço de Banach complexo, então, sob a norma $\|\cdot\|_{*}: X^{c} \rightarrow \mathbb{R}$ definida por

$$
\|(x, z)\|_{*}:=\max \{\|x\|,|z|\}
$$

$X^{c}$ é um espaço de Banach complexo.

Por questão de simplicidade, denotaremos $\|.\|_{*}$ apenas por $\|$.$\| ficando claro pelo contexto$ de qual norma se trata.

Se $X$ é um espaço de Banach complexo e $\mathcal{P}(X)$ e $\mathcal{P}\left(X^{c}\right)$ denotam respectivamente o espaço dos polinômios sobre $X$ e $X^{c}$ assumindo valores em $\mathbb{C}$, para cada $P=\sum_{k=0}^{m} Q_{k}$, onde $Q_{k} \in \mathcal{P}\left({ }^{k} X\right)$, seja $P^{c} \in \mathcal{P}\left(X^{c}\right)$, o polinômio $m$-homogêneo definido por

$$
P^{c}(x, z):=\sum_{k=0}^{m} Q_{k}(x) z^{m-k}
$$

Claramente, $P \mapsto P^{c}$ é uma aplicação linear de $\mathcal{P}(X)$ e $\mathcal{P}\left(X^{c}\right)$. Temos também o seguinte resultado. 
Proposição 4.3.4. Se $X$ é um espaço de Banach complexo, a aplicação $P \mapsto P^{c}$ de $\mathcal{P}(X)$ em $\mathcal{P}\left(X^{c}\right)$ satisfaz.

(i) $P_{1}^{c} \ldots P_{n}^{c}=\left(P_{1} \ldots P_{n}\right)^{c}, P_{1}, \ldots, P_{n} \in \mathcal{P}(X)$.

(ii) $\|P\|=\left\|P^{c}\right\|, P \in \mathcal{P}(X)$.

Demonstração. (i) Sem perda de generalidade, podemos supor, para cada $1 \leq k \leq n$, $P_{k}=\sum_{i=0}^{m} Q_{k i}$ onde $Q_{k i} \in \mathcal{P}\left({ }^{i} X\right)$. Então

$$
\begin{aligned}
\left(P_{1} \ldots P_{n}\right)^{c}(x, z) & =\sum_{i_{1}, \ldots, i_{n}=0}^{m} Q_{1 i_{1}}(x) \ldots Q_{n i_{n}}(x) z^{n m-i_{1}-\ldots-i_{n}} \\
& =\sum_{i_{1}, \ldots, i_{n}=0}^{m}\left(Q_{1 i_{1}}(x) z^{m-i_{1}}\right) \ldots\left(Q_{n i_{n}}(x) z^{m-i_{n}}\right) \\
& =\left(\sum_{i_{1}=0}^{m}\left(Q_{1 i_{1}}(x) z^{m-i_{1}}\right)\right) \ldots\left(\sum_{i_{n}=0}^{m}\left(Q_{n i_{n}}(x) z^{m-i_{n}}\right)\right) \\
& =P_{1}^{c}(x, z) \ldots P_{n}^{c}(x, z)
\end{aligned}
$$

como queríamos.

(ii) Fixado $P \in \mathcal{P}(X)$, claramente

$$
\sup _{\|(x, z)\| \leq 1}\left|P^{c}(x, z)\right| \geq \sup _{\|(x, z)\| \leq 1}\left|P^{c}(z x, z)\right| .
$$

Se fosse

$$
\sup _{\|(x, z)\| \leq 1}\left|P^{c}(x, z)\right|>\sup _{\|(x, z)\| \leq 1}\left|P^{c}(z x, z)\right|,
$$

existiria $\left\|\left(x_{0}, z_{0}\right)\right\| \leq 1$, satisfazendo

$$
\sup _{\|(x, z)\| \leq 1}\left|P^{c}(x, z)\right| \geq\left|P^{c}\left(x_{0}, z_{0}\right)\right|>\sup _{\|(x, z)\| \leq 1}\left|P^{c}(z x, z)\right| .
$$

Observando-se que a função $f(z):=P^{c}\left(x_{0}, z\right)$ é $\mathbb{C}$-diferenciável, pelo princípio do módulo máximo, existe $\left|z_{1}\right|=1$ satisfazendo

$$
\left|P^{c}\left(x_{0}, z_{1}\right)\right|=\left|f\left(z_{1}\right)\right|=\max _{|z| \leq 1}|f(z)| \geq\left|P^{c}\left(x_{0}, z_{0}\right)\right|
$$


Como $\left|z_{1}\right|=1$ e $\left\|x_{0}\right\| \leq 1$, segue-se $\left\|\left(x_{0}, z_{1}\right)\right\|=\left\|\left(\frac{x_{0}}{z_{1}}, z_{1}\right)\right\|=1$ e consequentemente

$$
\begin{aligned}
\sup _{\|(x, z)\| \leq 1}\left|P^{c}(z x, z)\right| & <\left|P^{c}\left(x_{0}, z_{0}\right)\right| \leq\left|P^{c}\left(x_{0}, z_{1}\right)\right| \\
& =\left|P^{c}\left(z_{1}\left(\frac{x_{0}}{z_{1}}\right), z_{1}\right)\right| \leq \sup _{\|(x, z)\| \leq 1}\left|P^{c}(z x, z)\right|,
\end{aligned}
$$

uma contradição. Portanto

$$
\left\|P^{c}\right\|=\sup _{\|(x, z)\| \leq 1}\left|P^{c}(x, z)\right|=\sup _{\|(x, z)\| \leq 1}\left|P^{c}(z x, z)\right| .
$$

Notando-se que $\left|P^{c}(z x, z)\right|=\left|z^{m} P^{c}(x, 1)\right|=\left|z^{m} P(x)\right|$ para todo $z \in \mathbb{C}, x \in X$, podemos escrever

$$
\left\|P^{c}\right\|=\sup _{\|(x, z)\| \leq 1}\left|P^{c}(z x, z)\right|=\sup _{\|(x, z)\| \leq 1}\left|z^{m} P(x)\right|=\sup _{\|x\| \leq 1}|P(x)|=\|P\| .
$$

Portanto $\left\|P^{c}\right\|=\|P\|$, como queríamos.

Teorema 4.3.5. Sejam $P_{1}, \ldots, P_{n}$ polinômios sobre um espaço de Banach complexo $X$ de graus $m_{1}, \ldots m_{n}$ respectivamente. Então

$$
\left\|P_{1}\right\| \ldots\left\|P_{n}\right\| \leq \frac{\left(m_{1}+\ldots+m_{n}\right)^{m_{1}+\ldots+m_{n}}}{m_{1}^{m_{1}} \ldots m_{n}^{m_{n}}}\left\|P_{1} \ldots P_{n}\right\| .
$$

Demonstração. Em virtude da proposição 4.3.4, a verificação pode ser feita supondo todos os polinômios homogêneos. A demonstração será feita por indução em $n$.

Se $n=1$ a conclusão é óbvia. Supondo a tese válida para $n=k>1$, sejam $P_{1}, \ldots, P_{k+1}$ com $P_{i} \in \mathcal{P}\left({ }^{m_{i}} X\right), 1 \leq i \leq k$.

Aplicando o lema $4.3 .2 \operatorname{com} p=1$ e $l=k$ obtemos

$$
\left\|P_{1} \ldots P_{k}\right\|\left\|P_{k+1}\right\| \leq \frac{\left(m_{1}+\ldots+m_{k+1}\right)^{\left(m_{1}+\ldots+m_{k+1}\right)}}{\left(m_{1}+\ldots+m_{k}\right)^{\left(m_{1}+\ldots+m_{k}\right)} m_{k+1}^{m_{k+1}}}\left\|P_{1} \ldots P_{k+1}\right\| .
$$

De acordo com a hipótese de indução,

$$
\left\|P_{1}\right\| \ldots\left\|P_{k}\right\| \leq \frac{\left(m_{1}+\ldots+m_{k}\right)^{\left(m_{1}+\ldots+m_{k}\right)}}{m_{1}^{m_{1}} \ldots m_{k}^{m_{k}}}\left\|P_{1} \ldots P_{k}\right\| .
$$


Consequentemente

$$
\begin{aligned}
\left\|P_{1}\right\| \ldots\left\|P_{k+1}\right\| & \leq \frac{\left(m_{1}+\ldots+m_{k}\right)^{\left(m_{1}+\ldots+m_{k}\right)}}{m_{1}^{m_{1}} \ldots m_{k}^{m_{k}}}\left\|P_{1} \ldots P_{k}\right\|\left\|P_{k+1}\right\| \\
& \leq \frac{\left(m_{1}+\ldots+m_{k+1}\right)^{\left(m_{1}+\ldots+m_{k+1}\right)}}{m_{1}^{m_{1}} \ldots m_{k+1}^{m_{k+1}}}\left\|P_{1} \ldots P_{k+1}\right\| .
\end{aligned}
$$

Exemplo 4.3.6. Se $X=l_{1}$ e $P_{1}, \ldots, P_{n}$ são como no exemplo 4.3 .3 ocorre a igualdade. Concluímos que a constante obtida no teorema 4.3.5 não pode ser melhorada em geral.

Corolário 4.3.7. Seja $\left\{A_{1}, \ldots, A_{n}\right\} \subset \mathcal{L}\left({ }^{m} X\right)$ onde $X$ um espaço de Banach sobre $\mathbb{C}$. Então

$$
\left\|A_{1}\right\| \ldots\left\|A_{n}\right\| \leq n^{n m} \sup _{\|u\| \leq 1}\left|A_{1}(u) \ldots A_{n}(u)\right| .
$$

Demonstração. Para cada $1 \leq r \leq m$, seja $\pi_{r}: X^{m} \rightarrow X$ definida por

$$
\pi_{r}\left(x_{1}, \ldots, x_{m}\right):=x_{r}, x_{1}, \ldots, x_{m} \in X
$$

Para cada $1 \leq k \leq n$ definimos a aplicação $\Lambda_{k}: \overbrace{X^{m} \times \ldots \times X^{m}}^{m} \rightarrow \mathbb{C}$, por

$$
\Lambda_{k}\left(u_{1}, \ldots, u_{m}\right):=A_{k}\left(\pi_{1}\left(u_{1}\right), \ldots, \pi_{m}\left(u_{m}\right)\right), u_{1}, \ldots, u_{m} \in X^{n}
$$

Claramente $\left\{\Lambda_{1}, \ldots, \Lambda_{n}\right\} \subset \mathcal{L}\left({ }^{m} X^{m}\right)$, e para cada $1 \leq k \leq n$,

$$
\Lambda_{k}(u, \ldots, u)=A_{k}\left(\pi_{1}(u), \ldots, \pi_{m}(u)\right)=A_{k}(u), u \in X^{m}
$$

Então, fixando-se a coleção $\left\{P_{1}, \ldots, P_{n}\right\} \subset \mathcal{P}\left({ }^{m} X^{m}\right)$, onde para cada $1 \leq k \leq n, P_{k}(u):=$ $\Lambda_{k} u^{m}=A_{k}(u), u \in X^{n}$, teremos

$$
\left\|P_{1} \ldots P_{n}\right\|=\sup _{\|u\| \leq 1}\left|A_{1}(u) \ldots A_{n}(u)\right| \quad \text { e }\left\|P_{k}\right\|=\left\|A_{k}\right\|, 1 \leq k \leq n .
$$

Aplicando-se o teorema anterior, obtemos

$$
\begin{aligned}
\left\|A_{1}\right\| \ldots\left\|A_{n}\right\| & =\left\|P_{1}\right\| \ldots\left\|P_{n}\right\| \\
& \leq n^{n m}\left\|P_{1} \ldots P_{n}\right\|=n^{n m} \sup _{\|u\| \leq 1}\left|A_{1}(u) \ldots A_{n}(u)\right|,
\end{aligned}
$$

como queríamos. 
Corolário 4.3.8. Sejam $X$ e $Y_{i}(1 \leq i \leq n)$ espaços de Banach complexos. Para operadores lineares limitados $T_{i}: X \rightarrow Y_{i},(1 \leq i \leq n)$ temos

$$
\left\|T_{1}\right\| \ldots\left\|T_{n}\right\| \leq n^{n} \sup _{\|u\| \leq 1}\left\{\left\|T_{1}(u)\right\| \ldots\left\|T_{n}(u)\right\|\right\}
$$

Demonstração. Fixemos uma coleção $\left\{f_{1}, \ldots, f_{n}\right\}$ em $B_{Y_{i}^{*}}$, arbitrária. Segue-se que a coleção $\left\{f_{1} \circ T_{1}, \ldots, f_{n} \circ T_{n}\right\} \subset X^{*}$ e aplicando-se o o corolário 4.3.7,

$$
\begin{aligned}
\left\|f_{1} \circ T_{1}\right\| \ldots\left\|f_{n} \circ T_{n}\right\| & \leq n^{n} \sup _{\|u\| \leq 1}\left|f_{1}\left(T_{1}(u)\right) \ldots f_{n}\left(T_{n}(u)\right)\right| \\
& \leq n^{n} \sup _{\|u\| \leq 1}\left\{\left\|T_{1}(u)\right\| \ldots\left\|T_{n}(u)\right\|\right\}
\end{aligned}
$$

De acordo com o teorema de Hahn-Banach, para cada $1 \leq k \leq n$,

$$
\left\|T_{k}\right\|=\sup _{g \in B_{Y_{k}^{*}}}\left\|g \circ T_{k}\right\|
$$

Como a coleção $\left\{f_{1}, \ldots, f_{n}\right\} \subset B_{Y_{i}^{*}}$ é arbitrária, concluímos

$$
\left\|T_{1}\right\| \ldots\left\|T_{n}\right\| \leq n^{n} \sup _{\|u\| \leq 1}\left\{\left\|T_{1}(u)\right\| \ldots\left\|T_{n}(u)\right\|\right\}
$$

No caso de um espaço de Banach real podemos obter uma versão do teorema 4.3.5.

Teorema 4.3.9. Sejam $P_{1}, \ldots, P_{n}$ polinômios sobre um espaço de Banach real $X$ de graus $m_{1}, \ldots m_{n}$ respectivamente. Então

$$
\left\|P_{1}\right\| \ldots\left\|P_{n}\right\| \leq 2^{m-1} \frac{m^{m}}{m_{1}^{m_{1}} \ldots m_{n}^{m_{n}}}\left\|P_{1} \ldots P_{n}\right\|,
$$

onde $m=m_{1}+\ldots+m_{n}$.

Demonstração. Seja $\widetilde{X}$ uma complexificação natural de $X$ e para cada $1 \leq i \leq n$ seja $\widetilde{P}_{i}$ a única extensão complexa de $P_{i}$ sobre $\widetilde{X}$. Temos por 4.3 .5

$$
\left\|P_{1}\right\| \ldots\left\|P_{n}\right\| \leq\left\|\widetilde{P}_{1}\right\| \ldots\left\|\widetilde{P}_{n}\right\| \leq \frac{m^{m}}{m_{1}^{m_{1}} \ldots m_{n}^{m_{n}}}\left\|\widetilde{P}_{1} \ldots \widetilde{P}_{n}\right\|
$$


Claramente $P_{1} \ldots P_{n}$ é um polinômio homogêneo de grau $m$ sobre $X$. Da unicidade da extensão complexa, temos

$$
\widetilde{P_{1} \ldots P_{n}}=\widetilde{P}_{1} \ldots \widetilde{P}_{n}
$$

Então, de acordo com o teorema 4.2.2

$$
\left\|\widetilde{P}_{1} \ldots \widetilde{P}_{n}\right\|=\left\|\widetilde{P_{1} \ldots P_{n}}\right\| \leq 2^{m-1}\left\|P_{1} \ldots P_{n}\right\|
$$

De 4.7 e 4.8 , decorre

$$
\begin{aligned}
\left\|P_{1}\right\| \ldots\left\|P_{n}\right\| & \leq \frac{m^{m}}{m_{1}^{m_{1}} \ldots m_{n}^{m_{n}}}\left\|\widehat{P_{1} \ldots P_{n}}\right\| \\
& \leq 2^{m-1} \frac{m^{m}}{m_{1}^{m_{1}} \ldots m_{n}^{m_{n}}}\left\|P_{1} \ldots P_{n}\right\| .
\end{aligned}
$$

Notando-se que o exemplo 4.3.6 é válido para $l_{1}$ real ou complexo, concluímos que a constante obtida no teorema 4.3.9 não é a melhor em geral. No caso complexo, o lema 4.3.2 é fundamental para que a constante obtida seja a melhor possível e não é conhecida relação semelhante para o caso real. Para mais detalhes sugerimos [5].

Corolário 4.3.10. Seja $\left\{A_{1}, \ldots, A_{n}\right\} \subset \mathcal{L}\left({ }^{m} X\right)$ onde $X$ um espaço de Banach sobre $\mathbb{R}$. Então

$$
\left\|A_{1}\right\| \ldots\left\|A_{n}\right\| \leq 2^{n m-1} n^{n m} \sup _{\|u\| \leq 1}\left|A_{1}(u) \ldots A_{n}(u)\right| .
$$

Demonstração. Procedendo-se como na demonstração de 4.3.7, é possível se obter uma a coleção $\left\{P_{1}, \ldots, P_{n}\right\} \subset \mathcal{P}\left({ }^{m} X^{m}\right)$ satisfazendo

$$
\left\|P_{1} \ldots P_{n}\right\|=\sup _{\|u\| \leq 1}\left|A_{1}(u) \ldots A_{n}(u)\right| \quad \text { e }\left\|P_{k}\right\|=\left\|A_{k}\right\|, 1 \leq k \leq n
$$

Então, aplicando-se o teorema anterior, obtemos

$$
\begin{aligned}
\left\|A_{1}\right\| \ldots\left\|A_{n}\right\| & =\left\|P_{1}\right\| \ldots\left\|P_{n}\right\| \\
& \leq 2^{n m-1} n^{n m}\left\|P_{1} \ldots P_{n}\right\|=2^{n m-1} n^{n m} \sup _{\|u\| \leq 1}\left|A_{1}(u) \ldots A_{n}(u)\right|,
\end{aligned}
$$

como queríamos. 


\subsection{Lema de Phelps}

O principal resultado deste capítulo é uma versão multilinear do seguinte teorema devido a R.Phelps.

Teorema 4.4.1. (Lema de Phelps) Dado um espaço de Banach $X$, sejam $f, g \in S_{X^{*}} e$ $0<\epsilon<1$. Se $S_{X} \cap \operatorname{ker} f \subset S_{X} \cap g^{-1}(]-\epsilon, \epsilon[)$ então $\|g-\alpha f\| \leq 2 \epsilon$ para algum $|\alpha|=1$.

Demonstração. Se $S_{X} \cap \operatorname{ker} f \subset S_{X} \cap g^{-1}(]-\epsilon, \epsilon[)$ então $\left\|g \uparrow_{\text {ker } f}\right\| \leq \epsilon$ e de acordo com o teorema de Hahn-Banach, existe $h \in X^{*}$ tal que

$$
\|h\|=\left\|g \uparrow_{\operatorname{ker} f}\right\| \leq \epsilon \text { e } h(x)=g(x) \text { para todo } x \in \operatorname{ker} f
$$

Então ker $f \subset \operatorname{ker}(g-h)$ e portanto existe $a \in \mathbb{K}$ tal que $g-h=a f$. Segue-se

$$
|1-| a\|=|\|g\|-\|a f\|| \leq\| g-a f\|=\| h \| \leq \epsilon .
$$

Fixando-se $\alpha=\frac{a}{|a|}$ decorre,

$$
\begin{aligned}
\|g-\alpha f\| & =\|g-a f+(a-\alpha) f\| \\
& \leq\|g-a f\|+|a-\alpha|\|f\| \leq \epsilon+|1-| a|| \leq 2 \epsilon
\end{aligned}
$$

como queríamos.

A partir de agora $X_{1}, \ldots, X_{n}$ denotarão espaços de Banach sobre um corpo $\mathbb{K}$ com respectivas esferas unitárias $S_{X_{1}}, \ldots, S_{X_{n}}$. Denotaremos um elemento arbitrário de $X_{1} \times \ldots \times X_{n}$ como $(x, y)$ onde $x=\left(x_{1}, \ldots, x_{n-1}\right) \in X_{1} \times \ldots \times X_{n-1}$ e $y \in X_{n}$.

Se $A \in \mathcal{L}\left(X_{1}, \ldots, X_{n}\right)$, para cada $x \in X_{1} \times \ldots \times X_{n-1}$, denotaremos por $A_{x} \in X_{n}^{*}$ o funcional definido por

$$
A_{x}(y):=A(x, y), y \in X_{n}
$$

Para cada $y \in X_{n}$ denotaremos por $A_{y} \in \mathcal{L}\left(X_{1}, \ldots, X_{n-1}\right)$ a aplicação multilinear definida por

$$
A_{y}(x):=A(x, y), x \in X_{1} \times \ldots \times X_{n-1} .
$$

Precisamos de mais uma definição para auxiliar nossa terminologia. 
Definição 4.4.2. Sejam $A, B \in \mathcal{L}\left(X_{1}, \ldots, X_{n}\right), \epsilon>0$ e $V \subset S_{X_{1}} \times \ldots \times S_{X_{n}}$. Definimos

$$
\begin{aligned}
& Z(A):=\left\{(x, y) \in S_{X_{1}} \times \ldots \times S_{X_{n}}: A(x, y)=0\right\} \\
& \epsilon(A):=\left\{(x, y) \in S_{X_{1}} \times \ldots \times S_{X_{n}}:|A(x, y)| \leq \epsilon\right\} \\
& V_{x}:=\left\{y \in S_{X_{n}}:(x, y) \in V\right\} ; \\
& V_{y}:=\left\{x \in S_{X_{1}} \times \ldots \times S_{X_{n-1}}:(x, y) \in V\right\} ; \\
& \Lambda_{x}(B, A, \epsilon):=\left\{\beta \in \mathbb{K}:\left\|B_{x}-\beta A_{x}\right\| \leq \epsilon\right\} \\
& \Lambda_{y}(B, A, \epsilon):=\left\{\alpha \in \mathbb{K}:\left\|B_{y}-\alpha A_{y}\right\| \leq \epsilon\right\}
\end{aligned}
$$

Lema 4.4.3. Sejam $A, B \in \mathcal{L}\left(X_{1}, \ldots, X_{n}\right)$ e $\epsilon>0$. Se $Z(A) \subset \epsilon(B)$ então $\Lambda_{x}(B, A, \epsilon) \neq \emptyset$ para todo $x \in S_{X_{1}} \times \ldots \times S_{X_{n-1}}$.

Demonstração. Se $Z(A) \subset \epsilon(B)$ então para cada $x \in S_{X_{1}} \times \ldots \times S_{X_{n-1}}$, vale

$$
\operatorname{ker} A_{x} \cap S_{X_{n}} \subset\left\{y \in S_{X_{n}}:|B(x, y)| \leq \epsilon\right\}
$$

Segue-se que $\left\|B_{x} \Upsilon_{\text {ker } A_{x}}\right\| \leq \epsilon$ e de acordo com o teorema de Hahn-Banach, existe $H \in X_{n}^{*}$ tal que $\|H\|=\left\|B_{x} \Upsilon_{\operatorname{ker} A_{x}}\right\| \leq \epsilon$ e $H(y)=B_{x}(y), y \in \operatorname{ker} A_{x}$.

Então ker $A_{x} \subseteq \operatorname{ker}\left(B_{x}-H\right)$ e portanto existe $\beta \in \mathbb{K}$ tal que $B_{x}-H=\beta A_{x}$. Decorre

$$
\left\|B_{x}-\beta A_{x}\right\|=\|H\| \leq \epsilon
$$

e consequentemente $\beta \in \Lambda_{x}(B, A, \epsilon)$.

Lema 4.4.4. Sejam $A, B \in \mathcal{L}\left(X_{1}, \ldots, X_{n}\right)$ e $\epsilon, \epsilon_{0}>0$. Se $Z(A) \subset \epsilon(B)$ então, para cada $x \in S_{X_{1}} \times \ldots \times S_{X_{n-1}}$ e $y \in S_{X_{n}}$ :

(i) Se $\epsilon<\epsilon_{0}$ então $\Lambda_{y}(B, A, \epsilon) \subset \Lambda_{y}\left(B, A, \epsilon_{0}\right)$.

(ii) Se $\alpha \in \Lambda_{y}(B, A, \epsilon)$ e $\beta \in \Lambda_{x}(B, A, \epsilon)$ então

$$
|\alpha-\beta||A(x, y)| \leq 2 \epsilon
$$

(iii) Se $\alpha \in \Lambda_{y}(B, A, \epsilon)$ e $r>0$ então $\overline{\mathcal{B}_{r}(\alpha)} \subset \Lambda_{y}\left(B, A, \epsilon+r\left\|A_{y}\right\|\right)$.

(iv) $\Lambda_{x}(B, A, \epsilon)$ e $\Lambda_{y}(B, A, \epsilon)$ são conjuntos fechados.

Demonstração. (i) Evidente. 
(ii) Se $\alpha \in \Lambda_{y}(B, A, \epsilon)$ e $\beta \in \Lambda_{x}(B, A, \epsilon)$ então

$$
\begin{aligned}
|\alpha-\beta||A(x, y)| & =|\alpha A(x, y)-B(x, y)+B(x, y)-\beta A(x, y)| \\
& \leq|\alpha A(x, y)-B(x, y)|+|B(x, y)-\beta A(x, y)| \\
& \leq\left\|B_{y}-\alpha A_{y}\right\|+\left\|B_{x}-\beta A_{x}\right\| \leq 2 \epsilon .
\end{aligned}
$$

(iii) Se $\alpha \in \Lambda_{y}(B, A, \epsilon)$ e $|\eta| \leq r$ então

$$
\left\|B_{y}-(\alpha+\eta) A_{y}\right\| \leq\left\|B_{y}-\alpha A_{y}\right\|+|\eta|\left\|A_{y}\right\| \leq \epsilon+r\left\|A_{y}\right\|
$$

Isso demonstra que $(\alpha+\eta) \in \Lambda_{y}\left(B, A, \epsilon+r\left\|A_{y}\right\|\right)$ e concluímos

$$
\overline{\mathcal{B}_{r}(\alpha)} \subset \Lambda_{y}\left(B, A, \epsilon+r\left\|A_{y}\right\|\right)
$$

(iv) Para cada $x \in S_{X_{1}} \times \ldots \times S_{X_{n-1}}$, a função $f_{x}: \mathbb{K} \rightarrow \mathbb{R}$ definida por

$$
f_{x}(\alpha):=\left\|B_{x}-\alpha A_{x}\right\|, \alpha \in \mathbb{K}
$$

é claramente contínua. Então $f^{-1}([0, \epsilon])=\Lambda_{x}(B, A, \epsilon)$ é fechado.

Analogamente se verifica que $\Lambda_{y}(B, A, \epsilon)$ é fechado.

Lema 4.4.5. Para todo $n \in \mathbb{N}$ existe $d_{n} \geq 1$ tal que para quaisquer $A, B \in \mathcal{L}\left(X_{1}, \ldots, X_{n}\right)$ com $\|A\|=\|B\|=1$ e $\epsilon>0$, se $Z(A) \subset \epsilon(B)$ então $\|B-\gamma A\| \leq d_{n} \epsilon$ para algum $\gamma \in \mathbb{K}$.

Demonstração. A demonstração será feita por indução sobre $n$. Sejam $A, B \in \mathcal{L}\left(X_{1}, \ldots, X_{n}\right)$ com $\|A\|=\|B\|=1$ e $\epsilon>0$ tais que $Z(A) \subset \epsilon(B)$.

Se $n=1$, procedendo-se como na demonstração do lema de Phelps 4.4.1, obtemos $d_{1}=1$.

Se $n>1$ suponha a tese verdadeira para $n-1$. Primeiramente demonstraremos que $\Lambda_{y}\left(B, A, d_{n-1} \epsilon\right) \neq \emptyset$ qualquer que seja $y \in S_{X_{n}}$.

Com efeito, dado $y \in S_{X_{n}}$, por $Z(A) \subset \epsilon(B)$ obtemos

$$
Z\left(A_{y}\right)=(Z(A))_{y} \subset(\epsilon(B))_{y}=\epsilon\left(B_{y}\right)
$$

Se $A_{y} \equiv 0$ então $Z\left(A_{y}\right)=S_{X_{1}} \times \ldots \times S_{X_{n-1}} \subset \epsilon\left(B_{y}\right)$. Consequentemente, para cada $\alpha \in \mathbb{K}$,

$$
\left\|B_{y}-\alpha A_{y}\right\|=\left\|B_{y}\right\| \leq \epsilon \leq d_{n-1} \epsilon
$$


e portanto $\Lambda_{y}\left(B, A, d_{n-1} \epsilon\right)=\mathbb{K}$.

Se $B_{y} \equiv 0$ então para todo $x \in S_{X_{1}} \times \ldots \times S_{X_{n-1}}$

$$
\left|B_{y}(x)-\left(d_{n-1} \epsilon\right) A_{y}(x)\right|=\left|d_{n-1} \epsilon\right||A(x, y)| \leq d_{n-1} \epsilon
$$

e portanto $d_{n-1} \epsilon \in \Lambda_{y}\left(B, A, d_{n-1} \epsilon\right)$.

Se $A_{y}$ e $B_{y}$ não forem identicamente nulas então

$$
Z\left(\frac{A_{y}}{\left\|A_{y}\right\|}\right)=Z\left(A_{y}\right) \subset \epsilon\left(B_{y}\right)=\frac{\epsilon}{\left\|B_{y}\right\|}\left(\frac{B_{y}}{\left\|B_{y}\right\|}\right) .
$$

De acordo com a hipótese de indução, existe $a \in \mathbb{K}$ tal que

$$
\left\|\frac{B_{y}}{\left\|B_{y}\right\|}-a \frac{A_{y}}{\left\|A_{y}\right\|}\right\| \leq d_{n-1} \frac{\epsilon}{\left\|B_{y}\right\|} .
$$

De forma equivalente,

$$
\left\|B_{y}-\left(a \frac{\left\|B_{y}\right\|}{\left\|A_{y}\right\|}\right) A_{y}\right\| \leq d_{n-1} \epsilon
$$

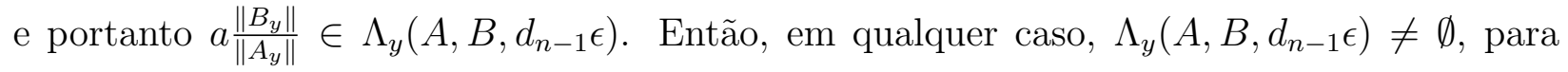
todo $y \in S_{X_{n}}$ e este fato terá uma importante aplicação a seguir.

De acordo com os corolários 4.3.7 e 4.3.10, existe uma constante $0<a_{n-1}<1$ tal que que para quaiquer $y_{1}, y_{2} \in S_{X_{n}}$

$$
a_{n-1}\left\|A_{y_{1}}\right\|\left\|A_{y_{2}}\right\| \leq \sup _{\|x\| \leq 1}\left|A_{y_{1}}(x) A_{y_{2}}(x)\right| .
$$

Dado $0<\delta<a_{n-1}$, pela hipótese de $\|A\|=1$, existe $y_{\delta} \in S_{X_{n}}$ tal que $1-\delta<\left\|A_{y_{\delta}}\right\| \leq 1$. Fixando-se $y \in S_{X_{n}}$, de acordo com a relação (4.9) acima, temos

$$
a_{n-1}\left\|A_{y_{\delta}}\right\|\left\|A_{y}\right\| \leq \sup _{\|x\| \leq 1}\left|A_{y_{\delta}}(x) A_{y}(x)\right|
$$

Supondo primeiramente $A_{y}$ não identicamente nula, deve existir $x \in S_{X_{1}} \times \ldots \times S_{X_{n-1}}$ satisfazendo

$$
\left(a_{n-1}-\delta\right)\left\|A_{y_{\delta}}\right\|\left\|A_{y}\right\|<\left|A\left(x, y_{\delta}\right) A(x, y)\right| .
$$

Evidentemente $A\left(x, y_{\delta}\right)$ e $A(x, y)$ são diferentes de zero. De acordo com o que foi discutido acima e em virtude do lema 4.4.3, podemos fixar $\alpha_{\delta} \in \Lambda_{y_{\delta}}\left(B, A, d_{n-1} \epsilon\right), \alpha \in \Lambda_{y}\left(B, A, d_{n-1} \epsilon\right)$ 
e $\beta \in \Lambda_{x}\left(B, A, d_{n-1} \epsilon\right)$. Pelo lema 4.4.4, item (ii), e pela relação (4.10) acima, temos

$$
\begin{aligned}
\left|\alpha-\alpha_{\delta}\right| & \leq|\alpha-\beta|+\left|\beta-\alpha_{\delta}\right| \\
& \leq \frac{2 d_{n-1} \epsilon}{|A(x, y)|}+\frac{2 d_{n-1} \epsilon}{\left|A\left(x, y_{\delta}\right)\right|} \\
& =2 d_{n-1} \epsilon\left(\frac{|A(x, y)|+\left|A\left(x, y_{\delta}\right)\right|}{\left|A(x, y) A\left(x, y_{\delta}\right)\right|}\right) \\
& <\frac{4 d_{n-1} \epsilon}{\left(a_{n-1}-\delta\right)\left\|A_{y}\right\|\left\|A_{y_{\delta}}\right\|}:=r .
\end{aligned}
$$

Então $\alpha_{\delta} \in \mathcal{B}_{r}(\alpha)$ e por $\alpha \in \Lambda_{y}\left(B, A, d_{n-1} \epsilon\right)$, o lema 4.4.4, item (iii), implica

$$
\begin{aligned}
\overline{\mathcal{B}_{r}(\alpha)} & \subset \Lambda_{y}\left(B, A, d_{n-1} \epsilon+r\left\|A_{y}\right\|\right) \\
& =\Lambda_{y}\left(B, A, d_{n-1} \epsilon+\frac{4 d_{n-1} \epsilon}{\left(a_{n-1}-\delta\right)\left\|A_{y_{\delta}}\right\|}\right) .
\end{aligned}
$$

Por $1-\delta<\left\|A_{y_{\delta}}\right\| \leq 1$, o lema 4.4.4, item $(i)$ implica

$$
\begin{aligned}
\alpha_{\delta} & \in \Lambda_{y}\left(B, A, d_{n-1} \epsilon+\frac{4 d_{n-1} \epsilon}{\left(a_{n-1}-\delta\right)\left\|A_{y \delta}\right\|}\right) \\
& \subset \Lambda_{y}\left(B, A, d_{n-1} \epsilon+\frac{4 d_{n-1} \epsilon}{\left(a_{n-1}-\delta\right)(1-\delta)}\right) \\
& =\Lambda_{y}\left(B, A,\left[1+\frac{4}{\left(a_{n-1}-\delta\right)(1-\delta)}\right] d_{n-1} \epsilon\right) .
\end{aligned}
$$

Se por outro lado, $A_{y} \equiv 0$ então, o lema 4.4 .4 , item $(i)$, implica

$$
\mathbb{K}=\Lambda_{y}\left(B, A, d_{n-1} \epsilon\right) \subset \Lambda_{y}\left(B, A,\left[1+\frac{4}{\left(a_{n-1}-\delta\right)(1-\delta)}\right] d_{n-1} \epsilon\right)
$$

Portanto

$$
\alpha_{\delta} \in \bigcap_{y \in S_{X_{n}}} \Lambda_{y}\left(B, A,\left[1+\frac{4}{\left(a_{n-1}-\delta\right)(1-\delta)}\right] d_{n-1} \epsilon\right):=K_{\delta}
$$

Em virtude do lema 4.4.4, item $(i v), K_{\delta}$ é interseção de conjuntos fechados, portanto fechado. Para verificarmos que $K_{\delta}$ é limitado, fixemos um elemento arbitrário $\alpha \in K_{\delta}$. Para 
todo $y \in S_{X_{n}}$ temos

$$
\begin{aligned}
|\alpha|\left\|A_{y}\right\|-\left\|B_{y}\right\| & \leq\left\|B_{y}-\alpha A_{y}\right\| \\
& \leq\left[1+\frac{4}{\left(a_{n-1}-\delta\right)(1-\delta)}\right] d_{n-1} \epsilon .
\end{aligned}
$$

Em particular, se $y=y_{\delta}$ então $\left\|A_{y_{\delta}}\right\|>0$ e

$$
|\alpha| \leq \frac{1}{\left\|A_{y_{\delta}}\right\|}\left(\left\|B_{y_{\delta}}\right\|+\left[1+\frac{4}{\left(a_{n-1}-\delta\right)(1-\delta)}\right] d_{n-1} \epsilon\right)
$$

Isso demonstra que $K_{\delta}$ é limitado. Então para todo $0<\delta<a_{n-1}, K_{\delta}$ é fechado e limitado, portanto compacto. Utilizando-se o lema 4.4.4, item $(i)$, demonstra-se que $K_{\delta} \subset K_{\delta^{*}}$ sempre que $\delta \leq \delta^{*}$. Consequentemente

$$
K:=\bigcap_{0<\delta<a_{n-1}} K_{\delta} \neq \emptyset
$$

Fixando-se $\gamma \in K$, temos

$$
\begin{aligned}
\|B-\gamma A\| & =\sup _{\|y\| \leq 1} \sup _{\|x\| \leq 1}|B(x, y)-\gamma A(x, y)| \\
& =\sup _{\|y\| \leq 1}\left\|B_{y}-\gamma A_{y}\right\| \\
& \leq\left(1+\frac{4}{a_{n-1}}\right) d_{n-1} \epsilon .
\end{aligned}
$$

A tese é obtida definindo-se

$$
d_{n}:=\left(1+\frac{4}{a_{n-1}}\right) d_{n-1}
$$

De acordo com o lema acima, para cada $n \in \mathbb{N}$ a constante obtida é

$$
d_{n}=\left(1+\frac{4}{a_{1}}\right) \ldots\left(1+\frac{4}{a_{n-1}}\right) .
$$


Usando as constantes $a_{k}$ obtidas nos corolários 4.3 .7 e 4.3.10 temos

$$
\begin{aligned}
& d_{n}=\prod_{k=1}^{n-1}\left(1+2^{2(k+1)}\right) \text { no caso complexo, } \\
& d_{n}=\prod_{k=1}^{n-1}\left(1+2^{4 k+1}\right) \text { no caso real. }
\end{aligned}
$$

Teorema 4.4.6. Para todo $n \in \mathbb{N}$ existe $D_{n}>0$ tal que para quaisquer $A, B \in \mathcal{L}\left(X_{1}, \ldots, X_{n}\right)$ com $\|A\|=\|B\|=1$ e $0<\epsilon<1 / d_{n}$, onde $d_{n} \geq 1$ é a constante obtida no lema anterior, se $Z(A) \subset \epsilon(B)$ então $\|B-\alpha A\| \leq D_{n} \epsilon$ para algum $|\alpha|=1$.

Demonstração. Sejam $A, B \in \mathcal{L}\left(X_{1}, \ldots, X_{n}\right)$ com $\|A\|=\|B\|=1$ e $\epsilon>0$ satisfazendo $Z(A) \subset \epsilon(B)$. Pelo lema 4.4.5 existe $\gamma \in \mathbb{K}$ tal que

$$
\begin{aligned}
|1-| \gamma|| & =|\|B\|-| \gamma|\|A\|| \\
& \leq\|B-\gamma A\| \leq d_{n-1} \epsilon .
\end{aligned}
$$

De acordo com as hipóteses, $\gamma \neq 0$. Fixando-se $\alpha:=\frac{\gamma}{|\gamma|}$ temos

$$
\begin{aligned}
\|B-\alpha A\| & \leq\|B-\gamma A+(\gamma-\alpha) A\| \\
& \leq\|B-\gamma A\|+|\gamma-\alpha|\|A\| \\
& \leq d_{n} \epsilon+|1-| \gamma|| \leq 2 d_{n} \epsilon .
\end{aligned}
$$

A tese é obtida definindo-se

$$
D_{n}:=2 d_{n}
$$




\section{Referências Bibliográficas}

[1] R. Aron, An introduction to polynomials on Banach spaces, Extracta Mathematicae, 17 (3) (2002), 303-329.

[2] R. Aron, C. Boyd, R. A. Ryan, I. Zalduendo, Zeros of polynomials on Banach spaces: the real story, Positivity, 7 (2003), 285-295.

[3] R. Aron, A. Cardwell, D. García, I. Zalduendo, A multilinear Phelps' lemma, Proc. Amer. Math. Soc., 135 (8) (2007), 2549-2554.

[4] R. Aron, L. Downey, M. Maestre, Zero sets and linear dependence of multilinear forms, Note di Mat., 1 (25) (2006), 49-54.

[5] C. Benítez, Y. Sarantopoulos, A. Tonge, Lower bounds for the norms of products of polynomials, Math. Proc. Cambridge Philos. Soc., 124 (1998), 395-408.

[6] E. Bishop, R. R. Phelps, A proof that every Banach space is subreflexive, Bull. Amer. Math. Soc., 67 (1961), 97-98.

[7] J. Bochnak, J. Siciak, Polynomials and multilinear mappings in topological vector spaces, Studia Mathematica, 39 (1971), 273-292.

[8] C. Costara. D. Popa, Exercices in Functional Analysis, Kluwer Academic Publishers, Dordrecht, 2003.

[9] S. Dineen, Complex Analysis on Infinite Dimensional Spaces, Springer Monographs in Mathematics, Springer-Verlag, London, 1999.

[10] M. Fabian, P. Habala, P. Hájek, V. M. Santalucía, J. Pelant, V. Zizler,, Functional Analysis and Infinite-Dimensional Geometry, Springer-Verlag, New York, 2001.

[11] J. Ferrer, On the zero-set of real polynomials in non-separable Banach spaces, Kyoto Univ., 43 (2007), 685-697. 
[12] J. Ferrer, Zeroes of real polynomials on $C(K)$ spaces, J. Math. Anal. Appl., 336 (2007), 788-796.

[13] L. Gillman, M. Jerison, Rings of Continuous Functions, Van Nostrand Reinhold, New York, 1960.

[14] A. Hissadomi, Propriedade de Dunford-Pettis Polinomial e Espaços Polinomialmente Schur, Dissertação de Mestrado, IME-USP, São Paulo, 1998.

[15] I. E. Leonard, J. H. M. Whitfield, A classical Banach space: $l_{\infty} \backslash c_{0}$, Rocky Mountain J. Math., 13 (3) (1983), 531-539.

[16] R. E. Megginson, An Introduction to Banach Space Theory, Graduate Texts in Mathemathics 183, Springer Verlag, New York, 1998.

[17] J. Mujica, Complex Analysis in Banach Spaces, North-Holland Math Studies vol 120, North-Holland, Amsterdan, 1986.

[18] G. A. Muñoz, Y. Sarantopoulos, A. Tonge, Complexifications of real Banach spaces, polynomials and multilinear maps, Studia Mathematica, 134 (1) (1999), 1-33.

[19] L. Pellegrini, Um Teorema de Hahn-Banach para Polinômios Homogêneos, Dissertação de Mestrado, IME-USP, São Paulo, 2001.

[20] R. R. Phelps, A representation theorem for bounded convex sets , Proc. Amer. Math. Soc., 11 (1960), 976-983.

[21] P. Pethe, N. Thakare, A note on the Dunford-Pettis property and a Schur property, Indiana Univ. J. Math, 27 (2) (1978), 91-92.

[22] A. Plichko, A. Zagorodnyuk, On automatic continuity and three problems of "the Scottish book" concerning the boundedness of polynomial functionals, J. Math. Anal. Appl., 220 (2) (1998), 477-494.

[23] A. E. Taylor, Additions to the theory of polynomials in normed linear spaces, Tôhoku Math. J., 44 (1938), 302-318.

[24] R. C. Walker, The Stone-Čech Compactification, Springer Verlag, New York, 1974. 